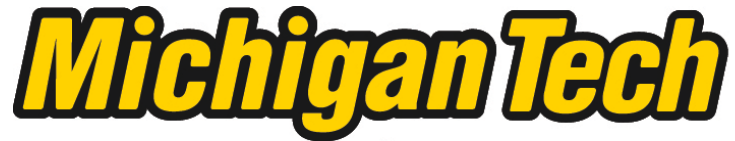 \\ Michigan Technological University Create the Future Digital Commons @ Michigan Tech
}

2015

\section{UNDERSTANDING ELECTRONIC STRUCTURE AND TRANSPORT PROPERTIES IN NANOSCALE JUNCTIONS}

Kamal B. Dhungana

Michigan Technological University

Follow this and additional works at: https://digitalcommons.mtu.edu/etds

Part of the Condensed Matter Physics Commons, and the Nanoscience and Nanotechnology

Commons

Copyright 2015 Kamal B. Dhungana

\section{Recommended Citation}

Dhungana, Kamal B., "UNDERSTANDING ELECTRONIC STRUCTURE AND TRANSPORT PROPERTIES IN NANOSCALE JUNCTIONS", Dissertation, Michigan Technological University, 2015.

https://doi.org/10.37099/mtu.dc.etds/954

Follow this and additional works at: https://digitalcommons.mtu.edu/etds

Part of the Condensed Matter Physics Commons, and the Nanoscience and Nanotechnology Commons 
UNDERSTANDING ELECTRONIC STRUCTURE AND TRANSPORT PROPERTIES

IN NANOSCALE JUNCTIONS

By

Kamal B. Dhungana

\begin{abstract}
A DISSERTATION
Submitted in partial fulfillment of the requirements for the degree of DOCTOR OF PHILOSOPHY

In Physics
\end{abstract}

MICHIGAN TECHNOLOGICAL UNIVERSITY

2015

(C) 2015 Kamal B. Dhungana 
This dissertation has been approved in partial fulfillment of the requirements for the Degree of DOCTOR OF PHILOSOPHY in Physics.

Department of Physics

Dissertation Advisor: Dr. Ranjit Pati

Committee Member: Dr. Ravindra Pandey

Committee Member: Dr. Maximilian Seel

Committee Member: $\quad$ Dr. Gregory Odegard

Department Chair: $\quad$ Dr. Ravindra Pandey 


\section{Contents}

List of Figures $\ldots \ldots \ldots \ldots \ldots \ldots \ldots \ldots \ldots \ldots \ldots$ vii

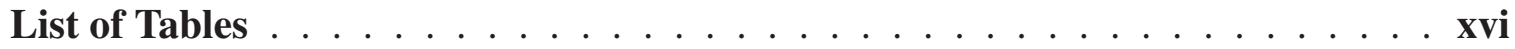

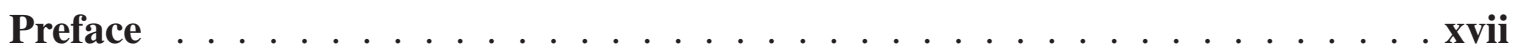

Acknowledgments $\ldots \ldots \ldots \ldots \ldots \ldots \ldots \ldots \ldots \ldots \ldots \ldots \ldots \ldots$ xvii

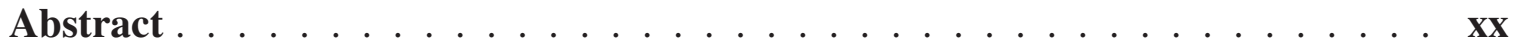

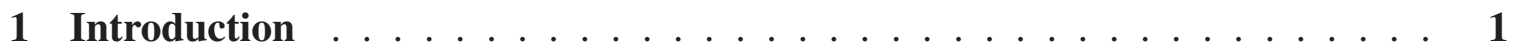

2 Density Functional Theory $\ldots \ldots \ldots \ldots \ldots$

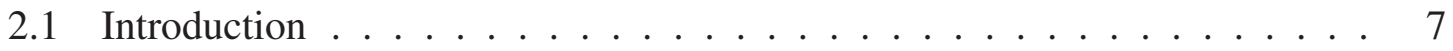

2.2 Many Body System $\ldots \ldots \ldots \ldots \ldots$

2.3 Hartree Product $\ldots \ldots \ldots \ldots \ldots$

2.4 Slater Determinant $\ldots \ldots \ldots \ldots \ldots \ldots$

2.5 Hartree-Fock Approximation $\ldots \ldots \ldots \ldots \ldots \ldots$

2.6 Density Functional Theory $\ldots \ldots \ldots \ldots$

2.7 Thomas-Fermi Model . . . . . . . . . . . . . . . . . . . . . 16 
2.8 The Hohenberg-Kohn Theorem . . . . . . . . . . . . . . . . . . . . 18

2.9 The Kohn-Sham Approach $\ldots \ldots \ldots$

2.9.1 Local Density Approximation (LDA) . . . . . . . . . . . . 24

2.9.2 Generalized Gradient Approximation (GGA) . . . . . . . . . 25

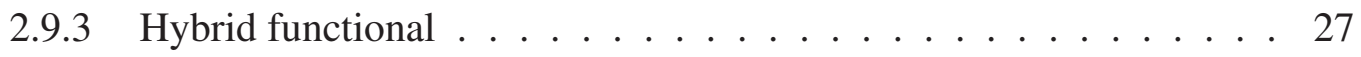

3 Theory of Quantum Transport _ . . . . . . . . . . . . . . . . . . . 29

3.1 Introduction . . . . . . . . . . . . . . . . . . . . . . . . . . . . 29

3.2 Characteristic Lengths . . . . . . . . . . . . . . . . . . 30

3.3 Modeling of nanoscale devices $\ldots \ldots \ldots$. . . . . . . . . . 33

3.3.1 Landauer formalism for electron transport . . . . . . . . . . . 33

3.3.2 Single particle Green's function approach $\ldots \ldots$. . . . . . 40

3.3.3 Spin-polarized electron transport . . . . . . . . . . . . . 45

4 Charge and Spin Transport in Molecular Junctions* . . . . . . . . . . 48

4.1 Introduction $\ldots \ldots \ldots \ldots \ldots \ldots \ldots \ldots$

4.2 Junction Dependent Conductance in a Molecular device . . . . . . . . . . 51

4.2.1 Computational Method . . . . . . . . . . . . . . . 53

4.2.2 I-V for Different Junction Geometries $\ldots \ldots \ldots \ldots$

4.2 .3 Conformational Change . . . . . . . . . . . . . . . . 58

4.2 .4 Transmission . . . . . . . . . . . . . . . . 60

4.2 .5 Conclusions . . . . . . . . . . . . . . . . . . 64

4.3 Molecular Spin-Valve Transistor . . . . . . . . . . . . . . . 65 
4.3.1 Modeling of a Spin-Valve Transistor . . . . . . . . . . . . . 66

4.3.2 Spin Polarized currents . . . . . . . . . . . . . . . . 67

4.3 .3 Transmission .................... 70

4.3.4 Conclusions ........................ 74

5 Electrical tuning of spin-current in a boron nitride nanotube quantum dot* . 75

5.1 Introduction . . . . . . . . . . . . . . . 75

5.2 Device modeling . . . . . . . . . . . . . . . 78

5.3 Spin Polarized Transport . . . . . . . . . . . . . . . . . 79

5.4 Conclusions . . . . . . . . . . . . . . . . . . 91

6 Fluorine Functionalized Boron Nitride Nanotube for Spintronics* . . . . . 93

6.1 Introduction . . . . . . . . . . . . . . . . 93

6.2 Computational Method . . . . . . . . . . . . . . . . . 96

6.3 Results and Discussions . . . . . . . . . . . . . . . . . . . . 98

6.3.1 Electronic structure . . . . . . . . . . . . . . . 98

6.3.2 Ferromagnetic spin ordering and thermodynamic stability . . . . . . 100

6.3.3 Current-Voltage characteristics . . . . . . . . . . . 101

6.3.4 Spin-injection efficiency and transmission . . . . . . . . . . . 102

6.3.5 Mechanism for spin filtering in F-BNNT . . . . . . . . . . . . . 108

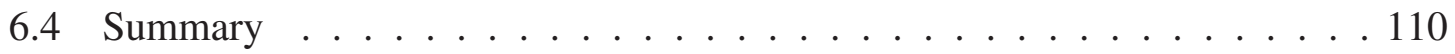

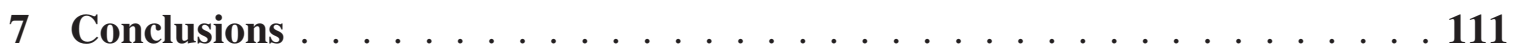




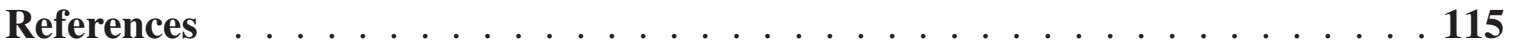

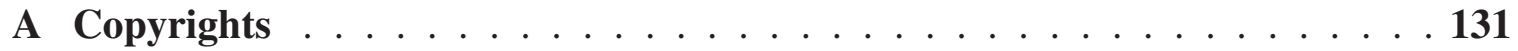

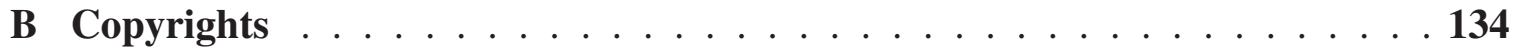

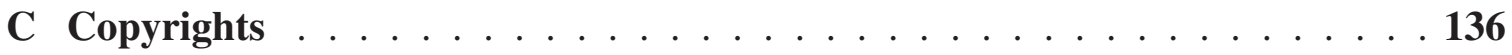




\section{List of Figures}

2.1 Mapping of a system of interacting particles to a system of non-interacting

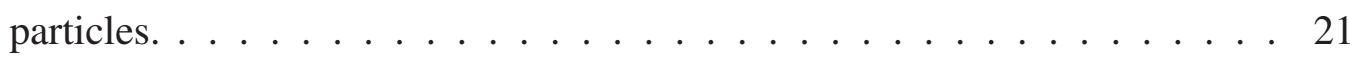

3.1 Schematic representation of a nano junction: the scattering region is connected to the reservoirs through left and right leads. . . . . . . . . . 34

4.1 Calculated current(I)-voltage(V) characteristics for Ru-bis(terpyridine) molecular wires with different junction configurations. Top and bottom panels show the junction structure and the corresponding I-V characteristic, respectively. The distance $d$ refers to the inter planar distance between the sulphur and the nearest gold; the $\mathrm{X}$ axis represents the direction of current flow. Reprinted figure with permission from K. B. Dhungana, S. Mandal, and R. Pati, J. Phys. Chem. C 116, 17268-17273 (2012). (C) The American Chemical Society. . . . . . . . . . . . . . . . . 56

(a) ONTOP-ONTOP $\ldots \ldots \ldots \ldots \ldots \ldots \ldots \ldots$

(b) ONTOP-ONTOP rotated $\ldots \ldots \ldots \ldots \ldots \ldots$

(c) HOLLOW-HOLLOW $\ldots \ldots \ldots \ldots \ldots \ldots \ldots$

(d) HOLLOW-HOLLOW rotated $\ldots \ldots \ldots \ldots$ 


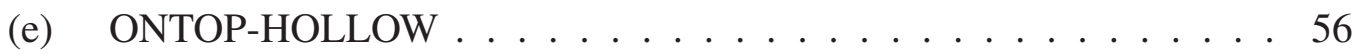

(f) ONTOP-HOLLOW rotated . . . . . . . . . . . . 56

4.2 Current-Voltage characteristics for Ru-bis(terpyridine) molecular wire with two different conformations of the molecular spacer; $\Phi$ represents the angle of rotation for one terpyridine plane from its equilibrium configuration about the current-flow axis. Reprinted figure with permission from K. B. Dhungana, S. Mandal, and and R. Pati, J. Phys. Chem. C 116, 17268-17273 (2012). (c) The American Chemical Society. . . . . . . . . . . . . . . . 59

4.3 Bias dependent transmission as a function of injection energy for interfacial distances of (a) 2.42 , (b) 2.82 , and (c) 3.02 respectively. The Fermi energy is set to zero in the energy scale; the dotted lines in each panel represent the chemical potential window. The eigen channels of the extended molecule are shown by solid vertical lines; L0 and H-2 refer to LUMO and HOMO-2, respectively. Reprinted figure with permission from K. B. Dhungana, S. Mandal, and R. Pati, J. Phys. Chem. C 116, 17268-17273 (2012). (c) The American Chemical Society. . . . . . . . . . . . . . . 61 
4.4 Participating bias dependent (a) occupied and (b) unoccupied molecular orbitals for strongly coupled $(d=2.42)$ and weakly coupled $(d=3.02)$ junctions. HOMO and LUMO refer to highest occupied molecular orbital and lowest unoccupied molecular orbital respectively. Reprinted figure with permission from Kamal B. Dhungana, S. Mandal, and and Ranjit Pati, J. Phys. Chem. C 116, 17268-17273 (2012). (C) 2012 American Chemical

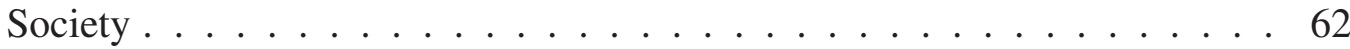

4.5 Schematic diagram of a molecular spin-valve transistor. Reprinted figure with permission from K. B. Dhungana and R. Pati, Appl. Phys. Lett. 104, 162404-162407 (2014). (c) 2014 AIP Publishing LLC. . . . . . . . . . . 66

4.6 Current $\left(I_{s d}\right)$-Voltage $\left(V_{s d}\right)$ characteristics in a Ru-bis-terpyridine molecular tunnel junction for parallel spin configuration (PC) and anti-parallel spin configuration ( APC) at different gate fields: (a) $\varepsilon_{g}=0.0 \mathrm{~V} / \AA$, (b) $\varepsilon_{g}=0.1$ $\mathrm{V} / \AA$, (c) $\varepsilon_{g}=0.3 \mathrm{~V} / \AA$, (d) $\varepsilon_{g}=0.4 \mathrm{~V} / \AA$. Reprinted figure with permission from K. B. Dhungana and R. Pati, Appl. Phys. Lett. 104, 162404-162407 (2014). (c) 2014 AIP Publishing LLC. . . . . . . . . . . . . . . . . . 68

4.7 (a) Spin-injection coefficient $(\eta)$ for the parallel spin configuration as a function of gate field $\left(\varepsilon_{g}\right) ; \eta$ is zero for the antiparallel spin configuration at low bias. (b) Bias $\left(V_{s d}\right)$ dependent TMR as a function of gate field. Reprinted figure with permission from K. B. Dhungana and R. Pati, Appl. Phys. Lett. 104, 162404-162407 (2014). (c) 2014 AIP Publishing LLC. . . . 70 
4.8 Gate field dependent spin polarized transmission at $V_{s d} \sim 0.3 \mathrm{~V}$ for the parallel (PC) and the anti-parallel (APC) spin configuration. (a) $\varepsilon_{g}=0.0$ $\mathrm{V} / \AA$, (b) $\varepsilon_{g}=0.4 \mathrm{~V} / \AA$, (c) $\varepsilon_{g}=0.0 \mathrm{~V} / \AA$, (d) $\varepsilon_{g}=0.4 \mathrm{~V} / \AA$. Notation: $\alpha$ stands for spin up and $\beta$ stands for spin down states. Reprinted figure with permission from K. B. Dhungana and R. Pati, Appl. Phys. Lett. 104, 162404-162407 (2014). (c) 2014 AIP Publishing LLC. . . . . . . . . . . . 71

4.9 Transmission plots for (a) PC and (b) APC at bias $\sim 1.8 \mathrm{~V}$ and $\varepsilon_{g}=0.4 \mathrm{~V} / \AA$. (c) Gate field dependent Stark shift associated with the Lowest Unoccupied Molecular orbital (LUMO) for PC and APC. Notation: $\alpha$ stands for spin up and $\beta$ stands for spin down states. Reprinted figure with permission from K. B. Dhungana and R. Pati, Appl. Phys. Lett. 104, 162404-162407 (2014). (c) 2014 AIP Publishing LLC. . . . . . . . . . . . . . . . . . 72

$5.1 I_{s d}-V_{s d}$ curves in a BNTQD tunnel junction for (a) PC and (b) APC as a function of $\varepsilon_{g}$. Insets show the schematic junction structures. (c) TMR vs. $\varepsilon_{g}$ at $V_{s d}$ of $0.2 \mathrm{~V}$. (d) Exchange coupling $(\mathbf{J})$ as function of $\varepsilon_{g}$. Inset shows the $\varepsilon_{g}$ dependent spin-profiles at the interfaces. The height and width of the arrow determine the magnitude of magnetic moment. Up and down arrows denote positive and negative magnetic moments respectively. Reprinted figure with permission from K. B. Dhungana and R. Pati, Phys. Chem. Chem. Phys. 16, 7996-8002 (2014). (c) The Owner Societies 2014. . . . . . 82 
5.2 (a) for PC and (b) for APC. The vertical dotted lines represent the planar atomic position of the device along the direction of current; the horizontal dotted line refers to the equilibrium situation; REP refers to the potential drop with respect to the equilibrium. Reprinted figure with permission from Kamal B. Dhungana and Ranjit Pati, Phys. Chem. Chem. Phys. 16, 7996-8002 (2014). (c) the Owner Societies 2014. . . . . . . . . . . 83

5.3 TMR as a function of applied bias $\left(V_{s d}\right)$ for (a) $\varepsilon_{g}=0.00 \mathrm{~V} / \AA$, and (b) $\varepsilon_{g}$ $=2.04 \mathrm{~V} / \AA$ A . Reprinted figure with permission from K. B. Dhungana and R. Pati, Phys. Chem. Chem. Phys. 16, 7996-8002 (2014). (c) The Owner Societies $2014 . \quad \ldots \ldots \ldots$. . . . . . . . . . . . . . . . . . . . . 84

5.4 Bias dependent spin injection factor $(\eta)$ in the BNTQD-Ni tunnel junction for $\varepsilon_{g}=0 \mathrm{~V} / \AA$ : (a) parallel spin configuration (PC), and (b) anti-parallel spin configuration (APC). Since $I_{d o w n}>I_{u p}$, the $\eta$ is found to be negative. Reprinted figure with permission from Kamal B. Dhungana and Ranjit Pati, Phys. Chem. Chem. Phys. 16, 7996-8002 (2014). (c) The Owner Societies

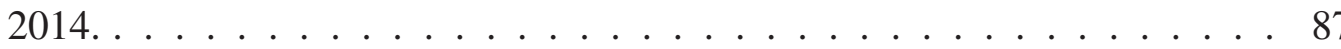

5.5 $\varepsilon_{g}$ dependent spin-polarized transmission for (a) parallel spin configuration and (b) anti-parallel spin configurations at $V_{s d}$ of $\sim 0.2 \mathrm{~V}$. Reprinted figure with permission from K. B. Dhungana and R. Pati, Phys. Chem. Chem. Phys. 16, 7996-8002 (2014). (c) The Owner Societies 2014. . . . . . . . . . 88 
5.6 (a) Bias dependent spin-polarized transmission for the APC of the extended system at $\varepsilon_{g}=2.04 \mathrm{~V} / \AA$. Dotted lines represent the chemical potential window $(\mathrm{CPW})$. The inset shows the bias dependent spin-down HOMO for the APC at $\varepsilon_{g}=2.04 \mathrm{~V} / \AA$. (b) Bias dependent Stark shift corresponding to the frontier spin-down orbitals as a function of $\varepsilon_{g}$. Upper two panels are for PC and lower two panels are for APC. H0, H1 refer to the HOMO and HOMO-1, and L0, L1 refer to the LUMO and LUMO+1 spin-down orbitals in the extended system. Reprinted figure with permission from K. B. Dhungana and R. Pati, Phys. Chem. Chem. Phys. 16, 7996-8002 (2014). (c) The Owner Societies 2014. . . . . . . . . . . . . . . . 91

6.1 Schematic diagram of a spin-filter with F-BNNTQD as the channel. $\Sigma_{l}$ and $\Sigma_{r}$ are the self energy functions that allow the F-BNNTQD to exchange energy and electrons with the semi-infinite gold electrodes of the open device. Reprinted figure with permission from K. B. Dhungana and R. Pati, J. Am. Chem. Soc. 136, 11494-11498 (2014). (C) The American Chemical

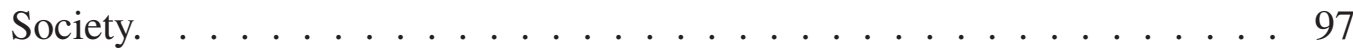

6.2 Electronic band structure of (a) pristine $(6,0)$ BNNT, (b) fluorinated $(6,0)$ BNNT (spin up states), and (c) fluorinated $(6,0)$ BNNT (spin down states); the Fermi-energy is set to zero. Reprinted figure with permission from K. B. Dhungana and R. Pati, Sensors 14, 17655-17686 (2014). (c) K. B. Dhungana and R. Pait. . . . . . . . . . . . . . . . . . . 99 
6.3 I-V characteristics of the pristine BNNTQD and F-BNNTQD coupled to the metallic gold electrodes; channel length is $\sim 1.5 \mathrm{~nm}$. Reprinted figure with permission from Kamal B. Dhungana and Ranjit Pati, J. Am. Chem. Soc. 136, 11494-11498 (2014). (c) The American Chemical Society. . . . . 102

6.4 the variation of the magnitude of spin injection coefficient $(\eta)$ with bias; channel length is $\sim 1.5 \mathrm{~nm}$; Up and Down refer to majority and minority spin states, respectively. Reprinted figure with permission from K. B. Dhungana and R. Pati, J. Am. Chem. Soc. 136, 11494-11498 (2014). (c) The American Chemical Society. . . . . . . . . . . . . . . . . 103

6.5 Spin dependent transmission in the F-BNNTQD-gold junction; the F-coverage is $4.1 \%$ and the channel length is $\sim 1.5 \mathrm{~nm}$; Up and Down refer to majority and minority spin states, respectively. Reprinted figure with permission from K. B. Dhungana and R. Pati, J. Am. Chem. Soc. 136, 11494-11498 (2014). (c) The American Chemical Society. . . . . . . . 104

6.6 Spin dependent transmission in the F-BNNTQD-gold junction for the channel length of $\sim 2 \mathrm{~nm}$ and F-coverage of $4.1 \%$; Up and Down refer to majority and minority spin states, respectively. Reprinted figure with permission from K. B. Dhungana and R. Pati, J. Am. Chem. Soc. 136, 11494-11498 (2014). (c) The American Chemical Society. . . . . . . . . . 105 
6.7 Spin dependent transmission in the F-BNNTQD-Au junction; F-coverage is $8.2 \%$ and channel length is $\sim 1.5 \mathrm{~nm}$; Up and Down refer to majority and minority spin states, respectively. Reprinted figure with permission from K. B. Dhungana and R. Pati, J. Am. Chem. Soc. 136, 11494-11498 (2014). (c) The American Chemical Society. . . . . . . . . . . . . . 106

6.8 Spin dependent transmission in F-BNNTQD-Au junction with a $12.5 \%$ defect; F-coverage is $8.2 \%$ and channel length is $\sim 1.5 \mathrm{~nm}$; Up and Down refer to majority and minority spin states, respectively. Reprinted figure with permission from K. B. Dhungana and Ranjit Pati, J. Am. Chem. Soc. 136, 11494-11498 (2014). (c) The American Chemical Society. . . . . . . . 107

6.9 Mechanism behind spin filtering in the F-BNNTQD. Horizontal lines represent the position of the discrete spin polarized energy levels of the magnetic F-BNNTQD. Solid smooth curve line represents the density of states (DOS) around the Fermi energy for the spin down states due to coupling with the gold electrodes; dotted line represents the DOS for the spin up states. (a) $\mathrm{V}=0 \mathrm{~V}$ (equilibrium situation), and (b) $\mathrm{V}>0 \mathrm{~V}$. Reprinted figure with permission from K. B. Dhungana and Ranjit Pati, J. Am. Chem. Soc. 136, 11494-11498 (2014). (c) The American Chemical Society. . . . . . . . . . . . . . . . . 109 
A.1 Copyright permission from the American Chemical Society for the article by K. B. Dhungana, S. Mandal, and R. Pati, J. Phys. Chem. C 116, 17268-17273(2012). . . . . . . . . . . . . . . 132

A.2 Copyright permission from the American Institute of Physics for the article by K. B. Dhungana and R. Pati, App. Phys. Lett. 104, 16204-17207 (2014). 133

B.1 Copyright permission from the American Chemical Society for the article by K. B. Dhungana and R. Pati, Phys. Chem. Chem. Phys. 16, 7996-8002

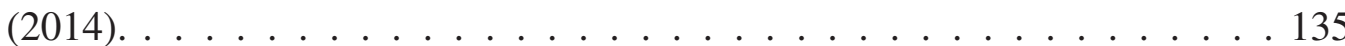

C.1 Copyright permission from the American Chemical Society for the article by K. B. Dhungana and R. Pati, J. Am. Chem. Soc. 136, 11494-11498

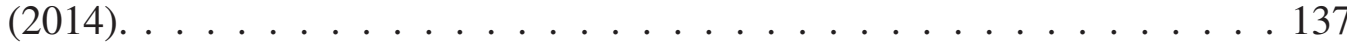




\section{List of Tables}

5.1 Components of dipole moment $(\alpha)$ and polarizability $(\beta)$ for parallel spin configuration (PC) and anti-parallel spin configuration (APC). Reprinted table with permission from K. B. Dhungana and R. Pati, Phys. Chem. Chem. Phys. 16, 7996-8002 (2014). (C) The Owner Societies 2014. . . . . . 90 


\section{Preface}

A part of this dissertation was published in peer reviewed journals. Chapter four is based on papers published in the Journal of Physical Chemistry C (Dhungana et al., 2012) and Applied Physics Letter (Dhungana el al., 2014). Chapter five is based on paper published in Physical Chemistry Chemical Physics (Dhungana el al., 2014). Similarly, chapter six is based on papers published in the Journal of American Chemical Society (Dhungana et al., 2014) and Sensors (Dhungana et al., 2014). For all these publications, my advisor, Professor Ranjit Pati, designed the projects and I did calculations. We analyzed the results together. I wrote all the papers with the suggestions and corrections from my advisor. We would like to thank Dr. Subhasish Mandal for helping us to analyze the results presented in the Journal of Physical Chemistry C (Dhungana et al., 2012). 


\section{Acknowledgments}

First and foremost, I would like to express my sincere gratitude to my advisor, Professor Ranjit Pati. His constant guidance and genuine enthusiasm for science kept me motivated throughout my $\mathrm{PhD}$ thesis work. Next, my sincere thanks goes to Professor Ravindra Pandey, Chair of the Department of Physics, for his invaluable suggestions and supports.

I would also like to thank Professor Maximilian Seel, Professor Ravindra Pandey, Professor Gregory M. Odegard, and Professor Ranjit Pati, my advisory committee members, for critically reviewing my work and giving me very helpful suggestions.

I had very fruitful discussions with my group members throughout the whole $\mathrm{PhD}$ period. I learned fundamental physics and several computational techniques from them. I would like to thank all present and past group members Partha, Subhasish, and Megnath.

Over the past five and a half years, I received help from several people from the Physics Department. I am thankful to all teaching and non-teaching staff members in the Physics Department. I am also thankful to Physics and IT Departments at MTU for providing me the computational facilities. Without these facilities, it would not be possible for me to finish my PhD on time. Also many friends in the Physics Department helped me tremendously for solving physics related problems and several technical issues that I encountered during my PhD. I am grateful to my friends Niraj, Douglas, Sanjaya, 
Madhusudan, Yong, Noopur, and Boyi for their helps. I would also like to thank Gowtham for maintaing the computing cluster at MTU and providing me the $\mathrm{AT}_{\mathrm{E} X t e m p l e t}$ for this thesis.

Without constant support from my family, it would be very difficult for me to complete my $\mathrm{PhD}$. I am immensely thankful to my parents, brothers, sister, and parents-in-law for their constant support, guidance, and inspiration. My wife, Jyoti, has helped me to resolve each and every issue I faced over the past three years; it has been possible for me to finish this thesis on time because of her unconditional support. My six month old little baby, Sanvi, is now my source of energy. Her every smile energizes me to focus and finish my work. In her every smile, I feel like she is telling "dad, finish your work first and then come to play with me". I dedicate this thesis to my family. 


\section{Abstract}

Understanding the electronic structure and the transport properties of nanoscale materials are pivotal for designing future nano-scale electronic devices. Nanoscale materials could be individual or groups of molecules, nanotubes, semiconducting quantum dots, and biomolecules. Among these several alternatives, organic molecules are very promising and the field of molecular electronics has progressed significantly over the past few decades. Despite these progresses, it has not yet been possible to achieve atomic level control at the metal-molecule interface during a conductance measurement, which hinders the progress in this field. The lack of atomic level information of the interface also makes it much harder for theorist to interpret the experimental results. To identify the junction configuration that possibly exists during the experimental measurement of conductance in molecular junction, we created an ensemble of Ruthanium-bis(terpyridine) molecular devices, and studied the transport behavior in these molecular junctions. This helps us identifying the junction geometry that yields the experimentally measured current-voltage characteristics.

Today's electronic devices mostly ignore the spin effect of an electron. The inclusion of spin effect of an electron on solid-state transistor allows us to build more efficient electronic devices; this also alleviates the problem of huge heat dissipation in the nanoscale electronic devices. Different materials have been utilized to build three terminals spin transistor since its inception in 1950. In search of suitable candidates for the molecular spin transistor, 
we have recently designed a spin-valve transistor based on an organometallic molecule; a large amplification (320\%) in tunnel magneto-resistance (TMR) is found to occur at an experimentally accessible gate field. This suggests that the organic molecules can be utilized for making the next generation three terminal spintronic devices. Similarly, we have designed a spin transistor based on boron nitride nanotube (BNNT) quantum dot. The TMR and exchange energy in BNNT based spin transistor are found to switch sign with the increase of the gate field.

The direct application of BNNT in electronic devices in several instances is hindered due to its large band gap. However, the functionalization of BNNT with different foreign species allows us to tune the band gap of BNNT. Fluorine functionalization in BNNT increases its conductance by more then 2 orders, as well as it induces strong magnetism in BNNT. The fluorine functionalization in BNNT thus has opened up the possibility of using the BNNT in future electronics and spintronics. Our study shows that a long range ferromagnetic spin ordering exists in the fluorinated BNNT even at a temperature much above the room temperature. Our spin polarized transport study further shows that the fluorine functionalization in BNNT not only enhances its conductance by more than two orders but also makes it a perfect spin filter with efficiency more than $99 \%$. Our transport study is based upon an orbital dependent density functional theory and a single particle Green's function approach. 


\section{Chapter 1}

\section{Introduction}

As anticipated from Moore's law, the size of a transistor in an integrated circuit is continuously shrinking [1]. Due to this continued progress in miniaturization of the transistor, it has been possible to realize smaller, faster, and cheaper electronic devices. Now, the size of the transistor is much smaller than $100 \mathrm{~nm}$. To come to this point, silicon industry has encountered several hurdles, and those hurdles have been successfully overcome with the various research breakthroughs and innovations by the research community [2]. The important issue right now is: how long can we sustain Moore's law in the future? It has been predicated that, by 2020, the size of the transistor would reach the physical limit, where the quantum tunneling behavior of an electron would degrade and ultimately destroy the switching feature of the transistor $[2,3]$. With the anticipation of this issue, researchers around the world are looking for alternative approaches for the 
continuation of scaling of electronic devices in the future. For example, researchers are trying to build nanoscale devices based on various semiconducting nano wires [4], quantum dots [5], and organic molecules [6, 7]. Among various candidate materials for future nanometer scale devices, organic molecules are found to be very promising because of the following reasons: (1) the electronic properties and functionalities of the molecules can span an extremely large range, and (2) they can be synthesized without flaws in the chemistry lab easily for a cheaper price [7]. The molecular electronics due to these advantages is viewed to be one of the best alternatives for the future nano-electronics. However, making a complete electronic circuit based on molecules is an extremely challenging task. One of the major challenges with the molecular electronics is to attach a molecule with macroscopic components in a circuit in a stable manner [8]. Despite several difficulties, molecular electronics has been progressing steadily since its inception $[6,7]$.

The history of molecular electronics started in 1974 when Aviram and Ratner first proposed an idea of using a molecule as a component of an electronic device [9]. It took more than two decades to measure the conductance successfully across a single molecular junction after the first theoretical prediction [10]. In 1997, Reed and colleagues, for the first time, invented the technique, which is known as break junction technique, to measure the conductance across a single molecular junction [10]. Later, the scanning tunneling microscopy (STM) technique is utilized to measure the conductance in the single molecular junction [11]. The STM technique is very popular and has been used extensively in recent years for building and measuring the conductance in single molecular junctions [7]. 
Over the past 40 years, a large number of molecules with novel properties have been tested for their potential application in future nano-electronics both theoretically and experimentally [7, 8]. Different molecules are found to exhibit interesting features, such as the Kondo effect [12], the Coulomb blockade effect [12, 13], and the negative differential resistance (NDR) behavior [14]. These novel features observed in different molecular systems make molecular electronics even more exciting.

All the molecular devices studied initially are of the two terminal type $[6,7]$. However, the ultimate goal of molecular electronics is to replace the silicon based three terminal field effect transistor (FET), where a gate field modulates the current between source and drain. Designing a three terminal molecular FET has been a longstanding challenge; practically, it is extremely difficult to place a gate electrode a few angstroms away from the molecule attached between source and drain [15]. Xu and colleagues in 2005, for the first time, introduced the electrochemical gate in the molecular junction, where they used perylene tetracarboxylic diimide molecule as a channel between the source and the drain. A large amplification in source-drain current was observed with the gate field [15]. Subsequently, using electro-migration technique, the three terminals molecular transistor based on 1, 8-octanedithiol (ODT) with an alkyl backbone has been reported by Song et al [16]. Very recently, Xiang et al. have made the molecular FET using the mechanically controllable break junction technique [17]. Along with the experiential progress, a number of theoretical approaches based on quantum mechanics, such as tight binding method $[18,19,20,21,22,23]$, and Green's function method $[24,25,26,27,28]$ have been 
developed in order to understand the transport properties in the molecular devices. These methods allow us to investigate the fundamental properties of the molecular device under a non-equilibrium condition $[24,25]$. A single particle Green's function method together with density functional theory has been very successful in understanding the experimentally observed features in molecular devices. In recent years, this method has been very popular and widely used to study transport properties in both two and three terminals molecular devices [26, 27, 28, 29].

Today's electronic devices are mostly based on transport, manipulation, and storage of electronic charges. The two major issues with the charge based conventional electronic devices are the consumption of enormous power and the production of immense heat during the device operation $[30,31,32,33]$. By including the spin effect of the electron in the device, we can overcome these difficulties. Moreover, the inclusion of spin effect of the electron in the transistor allows us to merge the data storage and the processing units into a single chip; as a result, spin electronics, called spintronics, allows us to miniaturize the electronic device significantly $[30,31,32,33]$. The idea of using a spin effect of the electron in the electronic device did not get much attention before the discovery of giant magneto-resistance (GMR) effect, by A. Fert and P. Grunberg in 1988 [34, 35]. In a GMR device, two magnetic layers are separated by a thin metallic layer, and the resistance of this device depends upon the relative magnetization of these two magnetic layers $[30,31,32$, 33]. This effect is known as GMR effect, and it has been implemented in a commercial data storage device within 15 years of its discovery. 
Instead of a normal metal, we also can use a thin semiconducting or insulating material between two magnetic layers to build a magnetoresistive device; this device is termed as tunnel magnetoresistive (TMR) device. In general, the TMR effect is larger than the GMR effect $[36,37]$. From an application point of view, devices having higher magnetoresistance (MR) are more desirable because they exhibit stronger sensitivity. Now, the current research focus is directed toward finding a suitable candidate that exhibits higher MR and is easy to integrate with the existing technology. Various semiconducting [38] and insulating materials $[36,37]$ have been used to build TMR devices over the past two decades. One of the most important properties of a material that determines its applicability in spintronics is the spin coherence length, which fundamentally depends upon hyperfine and spin-orbit interactions [39]. Organic molecules usually have very long spin coherence length because both the hyperfine and spin-orbit interactions are very weak in them [39]. The observed long spin-coherence length makes organic molecules more appealing for spintronics.

The field of molecular spintronics started after the successful measurement of magneto-resistance in a multi-wall carbon nanotube (CNT) coupled between two ferromagnetic electrodes by Tsukagoshi et al. in 1999; spin-coherence length of CNT was reported to be $130 \mathrm{~nm}$ by the authors [40]. Later, several experimental groups have investigated two and three terminal molecular scale spin-valve devices using STM and break junction techniques [41, 42, 43, 44, 45, 46] . Similarly, several theoretical groups have carried out the spin-polarized transport study in various molecular spin-valve devices using first-principles approaches [39, 47, 48, 49]. Currently, researchers are focusing on a 
three terminal molecular scale spin-valve transistor $[46,49,50]$; its success is expected to revolutionize modern electronics.

Alternatively, spin-polarized currents in the circuit can be generated by using a device consisting of a ferromagnetic channel between two normal metallic electrodes; this kind of device is known as a spin filtering device, and the channel material is called a spin-filtering material $[51,52,53,54,55]$. Organo-metallic magnetic molecules $[51,53,55,56,57,58]$ and magnetic insulators $[54,59,60]$ have been used in recent years for designing the spin-filtering devices. Materials that exhibit ferromagnetism at room temperature are highly desirable for making the room temperature spin filtering device and currently the researchers are looking for these materials $[52,61]$.

Here, using first-principles density functional theory and a single particle Green's function approach, we have studied spin-polarized and spin-unpolarized electron transport through Ru-bis-terpyridine molecule, and pristine and fluorine functionalized boron nitride nanotube (BNNT) quantum dots. We have also investigated the electronic structure and magnetic property of BNNT and fluorine functionalized BNNT.

The rest of the thesis is organized as follows. In Chapter 2, I discuss briefly the density function theory which is followed by quantum transport theory in nanoscale devices in Chapter 3. Results and discussion are described in Chapters 4, 5 and 6, followed by a brief conclusions in Chapter 7. 


\section{Chapter 2}

\section{Density Functional Theory}

\subsection{Introduction}

The density functional theory (DFT) is an alternative approach to the wave function based method for solving the Schrödinger wave equation (SWE) for a many body system [62, 63]. In terms of accuracy, the wave function based approach [64] may give better results than the DFT. However, in practice, it is almost impossible to solve the SWE for the system having relatively a large number of electrons by using the wave function based method due to the computational complexity [62]. This problem of solving the SWE for a bigger system can be overcome by using the density functional theory, where the electron density $n(r)$ is considered as the fundamental quantity from which all the quantum mechanical 
observables are calculated $[65,66,67,68,69,70,71]$. In recent years, it has become the most widely used computational tool in chemistry, physics, and material science, and the use of DFT is being extended to other disciplines $[72,73]$ as well. My aim in this chapter is to outline the basic ideas of DFT. Before I discuss the DFT, for completeness, I will briefly discuss about the time independent SWE for the many-body system and the wave function based Hartree-Fock approach $[63,64,74,75,76]$ for solving the SWE.

\subsection{Many Body System}

The time independent SWE for the many body system can be written as $[62,63,64,76]$ :

$$
\hat{H} \Psi\left(r_{1}, r_{2}, \ldots \ldots, r_{N}\right)=E \Psi\left(r_{1}, r_{2}, \ldots \ldots, r_{N}\right)
$$

where $\Psi\left(r_{1}, r_{2}, \ldots \ldots, r_{N}\right)$ is the many electron wavefunction, $E$ is the total energy, and $\hat{H}$ is

the Hamiltonian of the system having $N$ electrons. The Hamiltonian operator $\hat{H}$, with Born Oppenheimer approximation [77] which assume that the nuclei of the atoms are frozen, is the sum of kinetic energy, external potential, and the potential due to electron-electron interaction and can be written as [78]:

$$
\hat{H}=-\frac{1}{2} \sum_{i=1}^{N} \nabla_{i}^{2}+v(r)+\sum_{i}^{N} \sum_{j>i}^{N} \frac{1}{\left|r_{i}-r_{j}\right|} .
$$


Here, we have used atomic units, where $\mathrm{e}=\hbar=m_{e}=1$. The external potential $v(r)$ in Equation 2.2 is due to the interaction between electrons and atomic nuclei. Thus, $v(r)$ is given by [78]

$$
v(r)=-\sum_{i}^{N} \sum_{\alpha}^{M} \frac{Z_{\alpha}}{\left|r_{i}-R_{\alpha}\right|},
$$

where $M$ is the number of atoms in a given system and $R_{\alpha}$ is the co-ordinate of $\alpha^{\text {th }}$ atom.

The Hamiltonian operator in Equation 2.2 does not take into account the spin effect of electrons. Thus, the spin effect must be included in the wavefunction. This effect can be incorporated by considering an antisymmetic wavefunction, $i . e$, the wavefunction must be antisymmetric with respect to the interchange of coordinates of any two electrons [64].

\subsection{Hartree Product}

Before finding a solution for a $N$ interacting electron system, let us first consider a simpler system containing $N$ non-interacting electrons. The Hamiltonian for such a system is the sum of Hamiltonians of individual electron as [64]:

$$
\hat{H}=\sum_{i=1}^{N} \hat{h}(i),
$$

where $\hat{h}(i)$ is the operator describing the kinetic and potential energies of $i^{\text {th }}$ electron. The Hamiltonian in Equation 2.4 represents two different situations: (a) electron-electron 
interaction is completely ignored, (b) $\hat{h}(i)$ is the effective one electron hamiltonian which includes the effect of electron-electron interaction in average way [64].

Since the total Hamilton is a sum of one electron Hamiltonians, a wavefunction which is a simple product of individual electron wavefunctions $\left(\phi_{i}\right)$ must be the eigen function of the total Hamiltonian. The total wavefunction thus can be written as [64]:

$$
\Psi\left(r_{1}, r_{2}, r_{3}, \ldots, r_{N}\right)=\phi_{1}\left(r_{1}\right) \phi_{2}\left(r_{2}\right) \phi_{3}\left(r_{3}\right) \ldots \ldots . . \phi_{N}\left(r_{N}\right)
$$

and the total energy $E$ becomes [64]

$$
E=\sum_{i=1}^{N} \varepsilon_{i}
$$

where $\varepsilon_{i}$ is the energy of $i^{t h}$ orbital. The wavefunction of the form of Eq. 2.5 is called Hartree product.

\subsection{Slater Determinant}

Hartree product does not take into account the indistinguishability of electrons, and hence it does not fulfill the antisymmetric principle of wavefunction. The antisymmetric principle can be included in the wavefunction as mentioned in section 2.2 by considering the 
wavefunction as the slater determinant of spin orbitals as [63, 64]:

$$
\Psi\left(r_{1}, r_{2}, r_{3}, \ldots, r_{N}\right)=(N !)^{-\frac{1}{2}}\left|\begin{array}{cccc}
\phi_{i}\left(x_{1}\right) & \phi_{j}\left(x_{1}\right) & \ldots . & \phi_{N}\left(x_{1}\right) \\
\phi_{i}\left(x_{2}\right) & \phi_{j}\left(x_{2}\right) & \ldots . & \phi_{N}\left(x_{2}\right) \\
. & . & \ldots . & . \\
. & . & \ldots . & . \\
\phi_{i}\left(x_{N}\right) & \phi_{j}\left(x_{N}\right) & \ldots \ldots & \phi_{N}\left(x_{N}\right)
\end{array}\right|
$$

The factor $(N !)^{-\frac{1}{2}}$ is the normalization factor. Slater determinant has $N$ electrons occupying $N$ spin orbitals without specifying which electron is in which orbital. The rows in slater determinant are labelled by electrons, while the columns are labelled by spin-orbitals. The interchange of two rows corresponds to the interchange of co-ordinates of two electrons, which changes the sign of the determinant $[63,64]$. As a result, the criterion of antisymmetric principle of wave function is satisfied. When two electrons are in the same spin-orbital, then the two columns of the determinants will be same, which results the determinant value to zero. This implies that the two electrons can not be in the same spin-orbital, which is known as Pauli exclusion principle [64]. The slater determinant form of the wavefunction includes the exchange correlation; this implies that the motion of two electrons having parallel spin is correlated. But the electrons with opposite spin are not correlated [64]. 


\subsection{Hartree-Fock Approximation}

The Hartree-Fock approximation is based on the variational principle, which states that the ground state wave function is the one that gives the lowest possible energy [74]. In Hartree-Fock approximation, each electron is assumed to move in the average potential produced by all electrons in addition to the potential due to fixed nuclear charges [74]. It further assumes that the wave function can be approximated by a single Slater determinant made up of one spin orbital per electron as $[63,64,74,78]$ :

$$
\Psi_{H F}=\frac{1}{(N !)^{\frac{1}{2}}} \operatorname{det}\left[\phi_{1} \phi_{2} \phi_{3} \ldots \ldots . . \phi_{N}\right] .
$$

The Hartree-Fock energy $\left(E_{H F}\right)$ is given by $[63,64,74,75]$

$$
E_{H F}=<\Psi_{H F}|\hat{H}| \Psi_{H F}>=\sum_{i=1}^{N} H_{i}+\frac{1}{2} \sum_{i, j=1}^{N}\left(J_{i j}-K_{i j}\right)
$$

where

$$
\begin{gathered}
H_{i}=\int \phi_{i}^{*}(x)\left[-\frac{1}{2} \nabla_{i}^{2}+\sum_{\alpha=1}^{M} \frac{Z_{\alpha}}{r_{i \alpha}}\right] \phi_{i}(x) d x=\int \phi_{i}^{*}(x) h_{i} \phi_{i}(x) d x \\
J_{i j}=\iint \phi_{i}^{*}\left(x_{1}\right) \phi_{j}^{*}\left(x_{2}\right) \frac{1}{r_{12}} \phi_{i}\left(x_{1}\right) \phi_{j}\left(x_{2}\right) d x_{1} d x_{2} \\
K_{i j}=\iint \phi_{i}^{*}\left(x_{1}\right) \phi_{j}^{*}\left(x_{2}\right) \frac{1}{r_{12}} \phi_{i}\left(x_{2}\right) \phi_{j}\left(x_{1}\right) d x_{1} d x_{2}
\end{gathered}
$$


The two integrals $J_{i j}$ and $K_{i j}$ are called coulomb and exchange integrals, respectively. These integrals are real and satisfy the relation $J_{i j} \geq K_{i j} \geq 0$ [63].

The energy in Equation 2.9 is the function of spin-orbitals $\phi_{i}$. By varying the spin-orbital by an infinitesimal amount $\delta \phi_{i}$, we get a small variation in energy, and which is given by $[63,64,74,75]$

$$
\delta E=\sum_{i} \delta H_{i}+\frac{1}{2} \sum_{i, j}\left(\delta J_{i j}-\delta K_{i j}\right)
$$

In order that $E$ reach absolute minimum, it is necessary that $\delta E$ must be zero for any choice of $\delta \phi_{i}$. The spin-orbitals satisfy the orthonormality, i. e,

$$
\left\langle\phi_{i}^{*} \mid \phi_{i}\right\rangle=\delta_{i j}
$$

Now, we use the method of Lagrangian multiplier to find the minimum of energy with the constraint that the spin-orbitals are orthonormal. In this method, we first define a new function (called Lagrangian) as [64, 74, 75]:

$$
\mathscr{L}\left[\phi_{i}\right]=E_{H F}\left[\phi_{i}\right]-\sum_{i, j} \varepsilon_{i j}\left(\left\langle\phi_{i}^{*} \mid \phi_{i}\right\rangle-\delta_{i j}\right)
$$

Here, the quantity $\varepsilon_{i j}$ is called Lagrangian undetermined multiplier. Setting the first variation in $\mathscr{L}$ to zero, i.e., $\delta \mathscr{L}=0$, we get

$$
f(i) \phi_{i}(x)=\varepsilon_{i} \phi_{i}(x)
$$


where

$$
f(i)=-\frac{1}{2} \nabla_{i}^{2}-\sum_{\alpha} \frac{Z_{\alpha}}{r_{i \alpha}}+v^{H F}(i)
$$

The operator $f(i)$ is an effective one-electron operator, called the Fock operator. $v^{H F}(i)$ is the average potential experienced by the $i^{t h}$ electron due to the presence of the other electrons. This potential is called the Hartree-Fock potential [64].

Now, multiplying Equation 2.16 by $\phi_{i}^{*}(x)$ on both sides and integrating, we get

$$
\varepsilon_{i}=H_{i}+\sum_{j}\left(J_{i j}-K_{i j}\right)
$$

Taking summation on both side with respect to $i$ in Equation 2.18, we get

$$
E=\sum_{i} \varepsilon_{i}-\sum_{i<j}\left(J_{i j}-K_{i j}\right)
$$

Equation 2.19 gives the total electronic energy of a many electron system under HF approximation. The total energy is not same as the sum of the single electron energies: the contributions from the coulomb and exchange effects must be subtracted from the sum of the single electron energies in order to calculate the total energy in this approximation $[63,64,74,75]$.

Hartree-Fock approximation provides the exchange exactly; however, this approximation neglects more detailed correlations due to many body interactions. The effect of correlation 
is indeed not negligible $[62,74]$. The energy discrepancy between the exact non-relativistic energy $\left(\mathscr{E}_{0}\right)$ and the energy from H-F approximation $\left(E_{0}\right)$ is called correlation energy $\left(E_{\text {core }}\right)$, i. e., $E_{\text {core }}=\mathscr{E}_{0}-E_{0}$. Since the H-F energy is an upper bound to the exact energy, the correlation energy is negative $[64,74]$. Different methods, such as configuration interaction (CI), many-body perturbation approach, and many body Green's function approach, which are known as post-Hartree-Fock approaches, have been developed to account the correlation more accurately $[63,64]$.

The main problem with these wavefunction based methods is the requirement of very high computational resources [62]. For example, let us consider a real space representation of $\Psi$ on a mesh where each coordinate is discretized by using 30 mesh points. If there are $\mathrm{N}$ electrons in the wave function, then $3 \mathrm{~N}$ coordinates will be required to represent $\Psi$ [62]. As a result, $30^{3 N}$ values are required to describe $\Psi$ on the mesh. Using 30 points to represent a co-ordinate is not very much. To get the better result, we need to use more grid points. Thus, it is impossible to use the full many body wavefunction to solve a problem having more than few electrons [62]. On the other hand, the density of election, $n(r)$, is the function of only three co-ordinates. Thus, only $30^{3}$ values are required to represent $n(r)$ on the same mesh. The Kohn-Sham formulation of DFT, which is the focus of this work, additionally employs a set of single-particle orbitals; $N$ orbitals are used to build the density. As a consequence, $30^{3} \times N$ values are needed to represent the density on the same mesh [62]. Thus, DFT provides an alternative approach to the wavefunction based method for solving a reasonably big many body quantum system with viable computational resources [62]. 


\subsection{Density Functional Theory}

Density functional theory (DFT) allows one to replace complicated $N$ electron wave function by the much simpler electron density $[62,63]$. In this approach, the many body interacting problem is mapped to a much easier-to-solve non-interacting problem [62]. This methodology is applied in a large variety of fields to many different problems, with the ground-state electronic structure problem simply being the most common. The simple scheme of the density-functional approach can be summarized as [62]:

$$
n(r) \Rightarrow \Psi\left(r_{1}, r_{2}, \ldots \ldots, r_{N}\right) \Rightarrow v(r)
$$

$i$. e., knowledge of electron density $n(r)$ implies the knowledge of the wave function and the potential, and hence of all other observables of the systems. The idea of local electron density and dependency of ground state energy of many electron system on electron density was formulated initially by Thomas and Fermi in 1920 [63, 79].

\subsection{Thomas-Fermi Model}

Thomas-Fermi (T-F) Model [79] is based on the uniform distribution of electrons in an atom. To derive the kinetic energy as a function of electron density, they assumed that each 
$h^{3}$ of the phase space volume ( $\mathrm{h}$ is the planck's constant) is occupied by two electrons and the electrons are moving in an effective potential field that is determined by nuclear charge. The density of $\Delta N$ electrons in real space within a cube with a side $l$ is given by [63]

$$
n(r)=\frac{\Delta N}{v}=\frac{\Delta N}{l^{3}}
$$

The total kinetic energy in terms of electron density of an atom with this assumption becomes [63]

$$
T_{T F}[n]=2.871 \int n^{5 / 3}(r) d r
$$

This is the famous Thomas-Fermi kinetic energy functional, which is the function of the local electron density [79]. Adding classical electrostatic energies of electron-nucleus attraction and electron-electron repulsion to Equation 2.22, we get the total energy functional as [63]:

$$
E_{T F}[n(r)]=2.871 \int n^{5 / 3}(r) d r-Z \int \frac{n(r)}{r} d r+\frac{1}{2} \iint \frac{n\left(r_{1}\right) n\left(r_{2}\right)}{\left|r_{1}-r_{2}\right|} d r_{1} d r_{2}
$$

This is the energy functional of the T-F theory of atoms. The ground state energy can be obtained by minimizing the functional 2.23 with the restriction that

$$
N=\int n(r) d^{3} r
$$


Using the method of Lagrangian multiplier, as in Equation 2.15, we get the famous Thomas-Fermi equation [63]

$$
\mu_{F T}=\frac{\delta E_{F T}[n]}{\delta n(r)}=\frac{5}{3} \times 2.87 n^{\frac{2}{3}}(r)-\frac{z}{r}+\int \frac{n\left(r_{2}\right)}{\left|r-r_{2}\right|} d r_{2} .
$$

The Thomas-Fermi model provides reasonably good predictions for atoms. But the accuracy for atoms is not high as that with other methods since this is a oversimplified model. The predictions for more complex molecular and solid system according to this model are rather poor. Hohenberg and Kohn [65, 66] in 1964 proved two fundamental theorems which show that for the ground state the T-F model may be considered as an approximation to the density functional theory [63].

\subsection{The Hohenberg-Kohn Theorem}

Following the idea of using a density functional for solving the many-particle problem, Hohenberg and Kohn formulated their famous theorems, which turned out to be a breakthrough in the density functional formalism $[65,66]$. If the exact many-particle wavefunction is known then the density of electrons can be determined as [62, 63]:

$$
n(r)=N \int d^{3} r_{2} \ldots \int d^{3} r_{N}\left|\Psi\left(r, r_{2}, r_{3}, \ldots \ldots, r_{N}\right)\right|^{2}
$$


For $N$ electrons system, the external potential $v(r)$ determines the Hamiltonian of the system. Thus, $N$ and $v(r)$ determine all the properties for the ground state. But instead of $N$ and $v(r)$, Hohenberg-Kohn considered the electron density as the basic variable $[65,66]$. In their first theorem, they showed that the electron density determines the external potential $v(r)$, within a trivial additive constant [63]. The total energy, determined by the external potential, as a function of density and can be written as $[62,63]$ :

$$
E_{v}[n]=T[n]+V_{n e}[n]+V_{e e}[n]=\int n(r) v(r) d r+F_{H K}(n)
$$

where $T[n]$ and $V_{e e}[n]$ are universal functionals, independent of $v(r)$, and combining these two functionals, we get

$$
F_{H K}[n]=T[n]+V_{e e}[n]
$$

The second theorem by Hohenberg and Kohn is about the energy from variational principle [63]. This states that for a trial density $n^{\prime}(r)$, such that $n^{\prime}(r) \geq 0$ and $\int n^{\prime}(r) d r=N$,

$$
E_{0} \leq E_{v}\left[n^{\prime}\right]
$$

where $E_{v}\left[n^{\prime}\right]$ is the energy functional as in Equation 2.27. This is similar to the variational principle used for wavefunction based HF approximation. This theorem also justifies the variational principle used in T-F model [63]. Again, using the method of Lagrangian 
multiplier to minimize the energy in Equation 2.27, we get

$$
\delta\left\{E_{v}[n]-\mu\left[\int n(r) d r-N\right]\right\}=0
$$

which yields Euler-Lagrangian equation:

$$
\mu=\frac{\delta E_{v}[n]}{\delta n(r)}=v(r)+\frac{\delta F_{H K}[n]}{\delta n(r)}
$$

The quantity $\mu$ is the Lagrangian undetermined multiplier and known as the chemical potential. Equation 2.31 is the basic working equation of density functional theory [63]. The universal functional $F_{H K}$ is crucial for using the density functional theory in practice $[62,69,70]$. Theoretically, it should be possible to calculate all observables, since they all are functionals of density. But in practice, we do not know yet how to do this explicitly. Another problem is that the numerical minimization of $E_{v}[n]$ is very tough $[62,63]$.

\subsection{The Kohn-Sham Approach}

Kohn and Sham in 1965 formulated the density functional theory in a much simpler way and make the DFT possible to implement in practice $[67,69,70]$. They considered a system of non-interacting electrons to mimic the system of real interacting electrons, in such a way, that the density of non interacting electrons is the same as that of interacting 
electrons (Fig. 2.1). They introduced the concept of orbitals $\left(\phi_{i}\right)$, which are known as Kohn-Sham (K-S) orbitals, and are used to determine the electron density [63]. However, the fundamental variable, which determines all quantum mechanical observables, is the total electron density. The electron density in terms of K-S orbitals is given by $[63,69,70]$

$$
n(r)=\sum_{i}^{N}\left|\phi_{i}\right|^{2}
$$

The kinetic energy within this approach can be calculated from the K-S orbitals as [63]:

$$
T_{S}[n]=-\frac{1}{2} \sum_{i}^{N}<\phi_{i}\left|\nabla^{2}\right| \phi_{i}>
$$

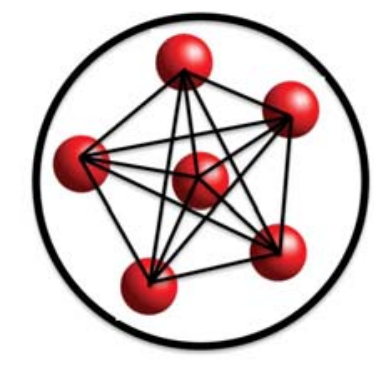

Interacting many body system

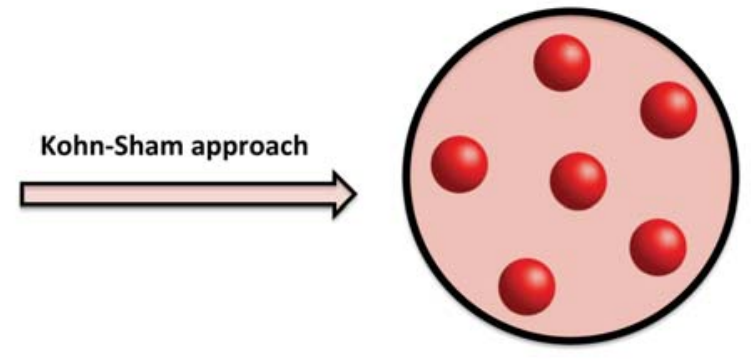

Non-interacting many body system

Figure 2.1: Mapping of a system of interacting particles to a system of non-interacting particles.

This is not a true kinetic energy, but it is the kinetic energy of non-interacting electrons. The correction required in kinetic energy due to this assumption is handled separately. The 
major contribution to the electron-electron interaction energy in any quantum mechanical system comes from the classical Coulomb interaction (or Hartree energy) [78]. Thus, the K-S theorem considers the Hartree energy as the electron-electron interaction energy. The Hartree energy in terms of density is given by [78]

$$
J[n]=\frac{1}{2} \iint \frac{n\left(r_{1}\right) n\left(r_{2}\right)}{\left|r_{1}-r_{2}\right|} d r_{1} d r_{2}
$$

The K.E in Equation 2.33 and electron-election interaction energy in Equation 2.34 are not accurate for the interacting systems. The sum of the error made in using a non-interacting kinetic energy and the error made in treating the electron-electron interaction classically is defined as the exchange-correlation energy $\left(E_{x c}[n]\right)$ in this approach $[62,63,78]$. Thus, the total energy functional can be written as [78]:

$$
E[n]=T_{s}[n]+\int v(r) n(r) d r+J[n]+E_{x c}[n]
$$

where the exchange energy is [63]

$$
E_{x c}[n]=\left(T[n]-T_{S}[n]\right)+\left(V_{e e}[n]-J[n]\right) .
$$

The energy functional in terms of Kohn-Sham orbitals can thus be written as [63]:

$$
E[n]=\sum_{i}^{N} \int \phi_{i}^{*}(r)\left(-\frac{1}{2} \nabla^{2}\right) \phi_{i}(r) d r+J[n]+E_{x c}[n]+\int v(r) n(r) d r
$$


K-S orbitals are orthonormal, thus

$$
\int \phi_{i}^{*}(r) \phi_{j}(r) d r=\delta_{i j}
$$

Minimizing the total energy using the method of Lagrange undetermined multiplier, we get,

$$
\delta\left[E[n]-\sum_{i}^{N} \sum_{j}^{N} \varepsilon_{i j} \int \phi_{i}^{*}(r) \phi_{j}(r) d r\right]=0
$$

Upon simplification, we get the final expression as $[63,78]$ :

$$
\left[-\frac{1}{2} \nabla^{2}+v_{e f f}\right] \phi_{i}(r)=\varepsilon_{i} \phi_{i}(r)
$$

where the effective potential $v_{e f f}$ is given by

$$
v_{e f f}(r)=v(r)+\int \frac{n\left(r^{\prime}\right)}{\left|r-r^{\prime}\right|} d r^{\prime}+v_{x c}(r)
$$

with the exchange-correlation potential $[63,78]$

$$
v_{x c}(r)=\frac{\delta E_{x c}[n]}{\delta n[r]}
$$

The Equation 2.40 is called K-S equation. The Kohn-Sham equation has the same structure as the Hartree-Fock equation with the non-local exchange potential replaced by the local exchange-correlation potential $v_{x c}$. The accuracy of the DFT based result depends upon 
the choice of exchange-correlation energy functionals $[63,62,78]$. Next, I will discuss different functionals which are widely used in recent years.

\subsubsection{Local Density Approximation (LDA)}

Local-density approximation is the most important type of approximation from the historical perspective; it is often called as the core of the modern DFT [62]. The exchange correlation energy, under this approximation, can be considered the same as that of a locally uniform electron gas of the same charge density $[62,78]$. This approximation is valid if the density of electron is slowly varying in a given region. The exchange correlation functional in terms of local charge density $\varepsilon_{x c}(\rho(r))$ can be written as [78]:

$$
E_{x c}[n]=\int n(r) \varepsilon_{x c}(\rho(r)) d r
$$

$\varepsilon_{x c}(\rho(r))$ can be separated into exchange and correlation contributions as [78]:

$$
\varepsilon_{x c}(\rho)=\varepsilon_{x}(\rho)+\varepsilon_{c}(\rho)
$$

The Dirac form can be used for $\varepsilon_{x}(\rho)[78]$;

$$
\varepsilon_{x}(\rho)=-C \rho^{1 / 3}
$$


where $C$ is a constant. The functional form of $\varepsilon_{c}(\rho)$ is not known exactly. Early approximate expressions for $\varepsilon_{x}(\rho)$ were based on perturbation theory; however, the modern expression of $\varepsilon_{x}(\rho)$ has been estimated for the homogeneous electron gas using the highly precise Quantum Monte Carlo method [78].

The LDA has proven to be an amazingly successful approximation. For many years the LDA has been applied to calculate the band structure, total energy, vibrational frequencies, elastic muduli, and phase stability in solid state physics [78]. The great success of this approximation is partly due to the systematic error cancellation: LDA underestimates correlation energy but overestimates the exchange energy, resulting in an unexpectedly good value of exchange-correlation energy[78]. However, it fails to provide accurate results for several quantum systems. For example, it overestimates the binding energy (typically by $20-30 \%$ ) of many systems, and the energy barriers in diffusive or chemical reactions may be too small or absent with this approximation $[62,78]$.

\subsubsection{Generalized Gradient Approximation (GGA)}

In the LDA, we use only the knowledge of density at point $r$ while constructing the exchange energy functional. In reality, the density of electron in any system is inhomogeneous [80]. To include the gradient effect of electron density, initially a gradient-expansion approximation (GEA), which is a series expansion of increasingly 
higher order density gradient terms, was developed. This approximation didn't help to improve the LDA result. However, the GEA provided the basis for the generalized gradient approximation (GGA) [78]. In the GGA [62, 81], instead of power-series-like systematic gradient expansion, one can consider more general functionals of $n(r)$ and $\Delta n(r)$. Such functionals are of the form

$$
E_{x c}^{G G A}[n]=\int d r f(n(r), \nabla n(r)) .
$$

The function $f(n, \Delta n)$ can be chosen in different ways; as a consequence, there exist various types of exchange energy functionals in GGA unlike in the LDA [78]. The most widely used GGA functionals in recent years are PBE (Perdew, Burke and Ernzerhof), and BLYP (Becke, Lee, Yang and Parr). Many other GGA-type functionals are also available, and new ones continue to appear $[69,70,78]$. Generally, GGA functionals seem to give reliable results for all type of chemical bonds [82]. Both GGA and LDA functionals, however, fail to give precise results for van der Waals interactions [83]. Both in physics and in chemistry the widespread use of GGA functionals has lead to major improvements as compared to LDA [78]. GGA functionals also fail to give the band gaps of insulator and many semiconductor correctly; these functionals usually underestimate their band gaps $[78,84]$. 


\subsubsection{Hybrid functional}

The quest for more accurate functionals beyond the GGA is still going on $[62,78]$. In recent years, the hybrid functionals, which incorporate exact exchange from Hartree-Fock theory with exchange and correlation from other functionals, are very popular in chemistry and physics, and can predict the results more accurately than other functionals in several systems [69, 70, 78]. For examples, the hybrid functionals predict the band gap of semiconductor much more accurately than LDA and GGA functionals [85]; also for molecular crystal, the band gap from hybrid functional is very close to the experimental result [86]. The hybrid approach sometime is called implicit density functional because the exact exchange energy is expressed in terms of the Kohn-Sham orbitals rather than density. One of the most popular hybrid functionals is B3LYP $[69,70]$. In B3LYP, the correlation is taken from LYP (Lee-Yang-Parr) GGA and the exchange is taken from Becke's three-parameter hybrid functional B3. Mathematically, B3LYP functional can be written as $[78,87]$ :

$$
E_{x c}^{B 3 L Y P}=E_{x c}^{L D A}+a_{0}\left(E_{x}^{H F}-E_{x}^{L D A}\right)+a_{x}\left(E_{x}^{G G A}-E_{x}^{L D A}\right)+a_{c}\left(E_{c}^{G G A}-E_{c}^{L D A}\right)
$$

where $a_{0}(=0.20), a_{x c}(=0.72)$, and $a_{c}(=0.81)$ are semi-empirical coefficients calculated from appropriate fitting of experimental data of atomization energies, ionization potentials, proton affinities, and total atomic energies. $E_{x c}^{L D A}, E_{x}^{L D A}$, and $E_{c}^{L D A}$ are respectively 
the exchange-correlation, exchange, and correlation energies obtained from the LDA approximation of DFT [78, 87]. $E_{H F}$ is the exact exchange energy estimated from the Hartree-Fock formulation. $E_{x}^{G G A}$ and $E_{c}^{G G A}$ are the exchange and correlation energies respectively calculated within the GGA approximation. 


\section{Chapter 3}

\section{Theory of Quantum Transport}

\subsection{Introduction}

My aim in this chapter is to describe the quantum transport approach that I adopted to study the spin-polarized and spin-unpolarized electron transport in various nanoscale junctions. The electron transport behavior in nanoscale devices is different from its bulk counterparts; the quantum nature of the electron plays the crucial role in determining the transport behavior in nanoscale devices $[7,24,25]$. Thus, unlike in semi-classical approaches, we need to incorporate the quantum effect of electron explicitly in the transport-modeling in order to understand the transport properties in nanoscale junctions $[24,25]$. In the first section of this chapter, I will discuss some length scales, which are fundamentally important 
for transport studies in nano-scopic physics. The second section in this chapter will be devoted to the modeling details of nanoscale junction, which will be divided into three subsections: (1) Landauer formalism for transport, (2) a single particle Green's function approach, and (3) the spin dependent transport.

\subsection{Characteristic Lengths}

Electron wavelength: The wave length of an electron is called de Broglie wavelength, and it is related to its kinetic energy. At low temperature, only those electrons which are very close to the Fermi-energy contribute to the transport, as a result the Fermi-wavelength of the electron is relevant to the transport [88]. The Fermi-wavelength is given by $[24,88]$

$$
\lambda_{f}=2 \pi / k_{f}=\sqrt{2 \pi / n_{s}}
$$

where $k_{f}$ is the Fermi wave vector and $n_{s}$ is the electron density for a 2-dimensional electron gas. The transport of the Fermi-electrons depends on the scattering potentials, which could be due to disorder or lattice vibrations (phonon) [88]. If the variations in this potential are comparable to the de Broglie (or Fermi) wavelength, then the quantum effects become more prominent [88].

Elastic mean free path: Elastic mean free path is the average distance travelled by an 
electron between two successive elastic scattering processes. Since the electron energy remains conserved during this process, the phase of the electron wave function is also expected to be conserved [88]. The transport through a channel is considered to be ballistic when the channel length is shorter than the elastic mean free path; the electron can freely move without being scattered. Elastic mean free path $\left(l_{m}\right)$ is given by $[24,25,88]$

$$
l_{m}=v_{f} \tau_{m}
$$

where $v_{f}$ and $\tau_{m}$ are the Fermi velocity and momentum relaxation time, respectively. Elastic mean free path varies from one material to another. The transport of electron beyond the elastic mean free path is called diffusive transport.

Phase coherence length: Energy relaxation and phase coherence are related with each other: dephasing cannot happen without energy relaxation. Phase coherence length of the electron is thus defined as the distance travelled by an electron between two successive inelastic scatterings. When a device size is longer than the phase coherence length, one should not expect the quantum interference effect within the device [24, 25, 88]. Thus, this length scale is crucial for determining the nature of transport in nanoscale devices. If the length of the device is shorter than the phase coherence length, the transport is considered to be coherent. At low temperature, one of the main sources of phase relaxation is the fluctuation of potential due to electron-electron interaction. Phase coherence length $\left(l_{\phi}\right)$ is defend as $[24,25,88]$ : 


$$
l_{\phi}=v_{f} \tau_{\phi}
$$

where $\tau_{\phi}$ is the phase relaxation time. Phase coherence length also varies from one material to another.

Spin diffusion length: Above mentioned length scales are related to the electronic charge state of the electron. Another length scale, known as spin diffusion length $\left(l_{s d}\right)$, is very important when we consider the spin polarized electron transport in nanoscale devices. $l_{s d}$ is the distance over which the traveling electron spin keeps the memory of its initial orientation; this depends upon the spin-orbit and hyperfine interactions [39]. Spin diffusion length in terms of spin relaxation time $\left(\tau_{\uparrow \downarrow}\right)$ and diffusion constant (D) can be written as [88]:

$$
l_{s d}=\sqrt{D \tau_{\uparrow \downarrow}}
$$

Usually, organic materials have relatively long spin coherence length. For example, spin coherence length in carbon nanotubes is reported to be $130 \mathrm{~nm}$ [40]. 


\subsection{Modeling of nanoscale devices}

\subsubsection{Landauer formalism for electron transport}

In this subsection, I am discussing an approach which is proved to be very effective in describing the transport in nanoscale devices [24]. This approach is known as Landauer (or scattering) approach $[89,90]$. Using this approach, we can express current through a nano junction in terms of transmission coefficient of an electron through it. This formalism is valid only for the coherent transport regime, where the device size is shorter than the phase coherence length of the electron $\left(l_{\phi}\right)$. In recent years, this is routinely applied to transport calculation through nano structures and single molecules. It is also well applicable in many semiconducting quantum dot systems with weak electron-electron interactions [25].

A schematic picture of a nanoscale junction is shown in Fig. 3.1. The entire device is partitioned into two parts: (1) central nanoscale regime, which is connected to the left and right electrodes through the quantum leads, and (2) the electrodes [25]. Here, we assume a coherent transport of the electron between the electrodes. The electron distribution in the central region is determined by the boundary conditions and is non-equilibrium at a finite bias $[24,25]$. However, the energy dissipation and phase loss of the electron due to inelastic scattering occurs at the electrodes [25]. Thus, the electron distributions in the electrodes 


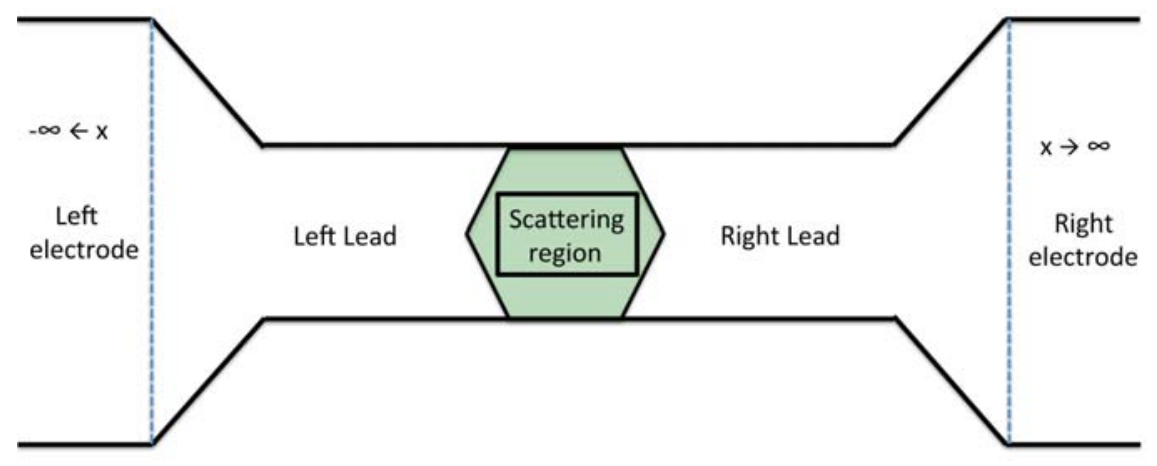

Figure 3.1: Schematic representation of a nano junction: the scattering region is connected to the reservoirs through left and right leads.

are at equilibrium $[24,25]$ and are given by Fermi-Dirac distribution as:

$$
f_{L, R}(E)=\frac{1}{e^{\left(E-\mu_{L, R}\right) / k_{B} T}+1}
$$

where $\mu_{L}$ and $\mu_{R}$ are the chemical potentials of left and right electrodes respectively at bias $V$.

The positions of chemical potentials in the left and right electrodes are determined by the applied bias. The bias $V$ is linked with the chemical potentials $\mu_{L, R}$ as [25]:

$$
V=\frac{\mu_{L}-\mu_{R}}{e}
$$

Here, the leads are assumed to be identical and the electrons are free to travel along the 
$\mathrm{x}$-axis (as shown in Figure 3.1) and are confined in $\mathrm{y}$ and $\mathrm{z}$ directions. Let us suppose that $H_{S}$ is the Hamiltonian of the central nanoscale regime and is given by [25]

$$
H_{S}=-\frac{\hbar^{2}}{2 m} \nabla^{2}+V(r)
$$

This Hamiltonian satisfy the following asymptotic conditions [25].

$$
\lim _{x \rightarrow-\infty} H_{S}=-\frac{\hbar^{2}}{2 m} \nabla^{2}+V_{L}(r) \equiv H_{L}
$$

and

$$
\lim _{x \rightarrow+\infty} H_{S}=-\frac{\hbar^{2}}{2 m} \nabla^{2}+V_{R}(r) \equiv H_{R}
$$

The Schrödinger wave equations (SWE) can be solved for the asymptotic Hamiltonians $H_{L}$ and $H_{R}$. The solutions for $H_{L}$ and $H_{R}$ are similar, and we just need to solve the SWE for one Hamiltonian. We can generalize the solutions for the other Hamiltonian. Here I am solving the SWE for $H_{L}$, which is given by

$$
\left[-\frac{\hbar^{2}}{2 m} \nabla^{2}+V_{L}(r)\right] \psi_{\alpha k}(r)=E_{\alpha}(k) \psi_{\alpha k}(r)
$$

The general solution of this equation has two components (transverse and longitudinal), which are independent to each other. Thus, the general solution is simply the product of these two components as [25]: 


$$
\psi_{\alpha k}(r)=\sqrt{\frac{1}{L_{x}}} u_{\alpha}\left(r_{\perp}\right) e^{i k x}, \quad-\infty<k<+\infty
$$

with energies

$$
E_{\alpha}(k)=\varepsilon_{\alpha}+\frac{\hbar^{2} k^{2}}{2 m}=\varepsilon_{\alpha}+\frac{1}{2} m v_{\alpha}^{2}
$$

There is a finite probability of the electron everywhere in space due to the traveling nature of eigen states 3.11. But, the exponentially decaying (evanescent) solutions of Equation 3.10 do not contribute to the probability density [25]. Similarly, we can obtain the eigen states and energies for $H_{R}$.

Now, for given $E$, the SWE for $H_{S}$ is

$$
\left[-\frac{\hbar^{2}}{2 m} \nabla^{2}+V(r)\right] \phi_{\alpha k}(r)=E \phi_{\alpha k}(r)
$$

The solutions $\phi_{\alpha k}(r)$ here can be determined by using the boundary conditions, $i . e_{.}, \phi_{\alpha k}(r)$ have to match asymptotically $(x \rightarrow \mp \infty)$ with the eigenstates of $H_{L}$ and $H_{R}$. There are traveling states both from left to right and from right to left at the central region [25]. First, we consider the traveling states from left to right.

Let us consider an electron having energy $E_{i}$ at $x \rightarrow-\infty$ region with the initial eigenstate $\psi_{i k_{i}}(r)$. The state of the electron in the center scattering region is complicated due to the complex form of potential $V(r)$. However, deep into the right electrode, it merely becomes a linear combination of eigenstates of the asymptotic Hamiltonian $H_{R}$ as [25]: 


$$
\phi_{i k_{i}}^{+}(r) \rightarrow \sum_{f=1}^{N_{R}} t_{i f} \psi_{f k_{f}}(r), \quad x \rightarrow+\infty
$$

where $N_{R}$ is the number of channels in the right lead at the given energy, and $t_{\text {if }}$ are the complex numbers. The symbol '+' with the wave function indicates that the wave function $\left(\phi_{i k_{i}}^{+}\right)$originates from the past wave function $\left(\psi_{i k_{i}}\right)$ [25]. The effect of evanescent wave function of $H_{S}$ in the deep right has zero effect. The electron state in the deep left lead is not simply the incident wave but it also has some back scattered states from the junction. Thus, the electron state in the deep left lead is

$$
\phi_{i k_{i}}^{+}(r) \rightarrow \psi_{i k_{i}}(r)+\sum_{f=1}^{N_{L}} r_{i f} \psi_{f k_{f}}(r), \quad x \rightarrow-\infty
$$

$N_{L}$ is the number of channels in the left lead, and $r_{i f}$ is the complex number.

Now, we calculate the current across the given surface $S$, which is perpendicular to the $\mathrm{x}$-axis (direction of current flow), carried by the wave function $\phi_{i k_{i}}^{+}(r)$. We use the current density operator $\hat{j}(r)$ to calculate current density $j(r)$ as [25]:

$$
j(r)=<\phi_{i k_{i}}^{+}|\hat{j}(r)| \phi_{i k_{i}}^{+}>=\frac{\hbar}{2 i m}\left[\left[\phi_{i k_{i}}^{+}(r)\right]^{*} \frac{\partial \phi_{i k_{i}}^{+}(r)}{\partial x}-\phi_{i k_{i}}^{+}(r) \frac{\partial\left[\phi_{i k_{i}}^{+}(r)\right]^{*}}{\partial x}\right]
$$

which upon integration along the y-z plane gives the average current $I\left(E_{i}\right)$ carried by the state at energy $E_{i}$ and is given by 
$I\left(E_{i}\right)=e<\phi_{i k_{i}}^{+}|\hat{I}| \phi_{i k_{i}}^{+}>=\frac{e \hbar}{2 i m} \int_{-\infty}^{+\infty} d y \int_{-\infty}^{+\infty} d z\left[\left[\phi_{i k_{i}}^{+}(r)\right]^{*} \frac{\partial \phi_{i k_{i}}^{+}(r)}{\partial x}-\phi_{i k_{i}}^{+}(r) \frac{\partial\left[\phi_{i k_{i}}^{+}(r)\right]^{*}}{\partial x}\right]=\frac{v_{i}\left(k_{i}\right)}{L_{x}}$

We assume a steady state current in the system. Thus, this current does not depend on the position of the surface we considered. Deep into the left lead it becomes [25]

$$
I_{L}\left(E_{i}\right)=I_{i}\left(E_{i}\right)\left(1-\sum_{f=1}^{N_{L}} R_{i f}\left(E_{i}\right)\right)
$$

where the quantity $R_{i f}\left(E_{i}\right)$ is called the reflection coefficient and is given by

$$
R_{i f}\left(E_{i}\right)=\left|r_{i f}\right|^{2} \frac{\left|I_{f}\left(E_{i}\right)\right|}{\left|I_{i}\left(E_{i}\right)\right|}
$$

The expression of $I_{i}\left(E_{i}\right)$ is similar to Equation 3.17 with $\phi_{i k_{i}}^{+}$is substituted by $\psi_{i k_{i}}$. Similarly, deep into the right lead, current becomes [25]

$$
I_{R}\left(E_{i}\right)=I_{i}\left(E_{i}\right) \sum_{f=1}^{N_{R}} T_{i f}\left(E_{i}\right)
$$

with transmission coefficient $T_{i f}\left(E_{i}\right)$ as:

$$
T_{i f}\left(E_{i}\right)=\left|t_{i f}\right|^{2} \frac{\left|I_{f}\left(E_{i}\right)\right|}{\left|I_{i}\left(E_{i}\right)\right|}
$$

The currents in the deep left and right leads must be same due to the steady state condition of current. If we consider the initial state $\psi_{i k_{i}}(r)$ from the right electrode, we will obtain the 
similar expression of currents at two different leads due to transmitted and reflected states [25].

Now, we are at the final stage of calculating the total current through the entire channels. The distributions of electrons in two different electrodes are different since the left and right electrodes are at two different chemical potentials $\left(\mu_{L, R}\right)$ in the presence of bias. Here the channels are assumed to be independent. Thus, the total current is the sum of all currents carried by all channels at all energies, and is given by [25]

$$
I=2 e \int d E\left[\sum_{i=1}^{N_{L}} \sum_{f=1}^{N_{R}} f_{L}(E) D_{i}\left(E_{i}\right) I_{i}\left(E_{i}\right) T_{i f}\left(E_{i}\right)-\sum_{i=1}^{N_{R}} \sum_{f=1}^{N_{L}} f_{R}(E) D_{i}\left(E_{i}\right) I_{i}\left(E_{i}\right) T_{i f}\left(E_{i}\right)\right]
$$

We introduced a factor 2 in Equation 3.22 to account the spin degree of freedom of the electron. The density of state for 1-D lead is given by

$$
D_{i}\left(E_{i}\right)=\frac{L_{x}}{2 \pi \hbar v_{\alpha}}
$$

The transmission coefficients from $\mathrm{R} \rightarrow \mathrm{L}$ and $\mathrm{L} \rightarrow \mathrm{R}$ in terms of transmission probabilities are defined as [25]:

$$
\begin{array}{ll}
T_{R L}(E)=\sum_{i=1}^{N_{R}} \sum_{f=1}^{N_{L}} T_{i f}(E), & R \rightarrow L \\
T_{L R}(E)=\sum_{i=1}^{N_{L}} \sum_{f=1}^{N_{R}} T_{i f}(E), & L \rightarrow R
\end{array}
$$


Particle flux must be conserved during the transport. Thus,

$$
T_{L R}(E)=T_{R L}(E)=T(E)
$$

With the substitution of density of state, and $I_{i}\left(E_{i}\right)$ in Equation 3.22, we get the final expression of current in terms of transmission coefficient as [25]:

$$
I=\frac{e}{\pi \hbar} \int_{-\infty}^{\infty} d E\left[f_{L}(E)-f_{R}(E)\right] T(E)
$$

\subsubsection{Single particle Green's function approach}

Just in the previous subsection, I have derived an expression of current through a ballistic conductor in terms of transmission function by using the Landauer approach [89, 90]. In this subsection, I will discuss how to calculate the transmission function in the real nanoscale junction using a single particle Green's function approach [24, 25], which has been adopted for the electron transport study here. As mentioned earlier, the entire heterogeneous nanoscale device is partitioned into three regions, namely left electrode, right electrode, and the central scattering region including leads. The two electrodes do not directly interact with each other; however, they couple with the center scattering region through the coupling potentials. Thus, the total Hamiltonian of the device is the sum of individual Hamiltonians of three regions and coupling potentials as [24, 25, 87]: 


$$
H=H_{L}+H_{R}+H_{S}+V_{L S}+V_{L S}^{\dagger}+V_{S R}+V_{S R}^{\dagger}
$$

where $H_{L}, H_{R}$, and $H_{S}$ are respectively the Hamiltonians of the left electrode, the right electrode, and the middle region. The terms $\left(V_{L S}+V_{L S}^{\dagger}\right)$ and $\left(V_{R S}+V_{R S}^{\dagger}\right)$ are the coupling potentials of the middle region with the left and right electrodes, respectively [87]. Considering the single particle wave functions $\Phi_{L}, \Phi_{R}$, and $\Phi_{S}$ are respectively the eigen functions of $H_{L}, H_{R}$, and $H_{S}$, we can write the Schrödinger wave equation for the entire device in the matrix form as $[24,25,87]$ :

$$
\left(\begin{array}{ccc}
H_{L} & V_{L S} & 0 \\
V_{L S}^{\dagger} & H_{S} & V_{S R}^{\dagger} \\
0 & V_{S R} & H_{R}
\end{array}\right)\left(\begin{array}{l}
\mid \Phi_{L}> \\
\mid \Phi_{S}> \\
\mid \Phi_{R}>
\end{array}\right)=E\left(\begin{array}{l}
\mid \Phi_{L}> \\
\mid \Phi_{S}> \\
\mid \Phi_{R}>
\end{array}\right)
$$

We get the following three equations upon multiplication of matrices in Equation 3.29:

$$
\begin{gathered}
H_{L}\left|\Phi_{L}>+V_{L S}\right| \Phi_{S}>=E \mid \Phi_{L}> \\
V_{L S}^{\dagger}\left|\Phi_{L}>+H_{S}\right| \Phi_{S}>+V_{S R}\left|\Phi_{R}>=E\right| \Phi_{S}> \\
V_{S R}\left|\Phi_{S}>+H_{R}\right| \Phi_{R}>=E \mid \Phi_{R}>
\end{gathered}
$$

Solving Equations 3.30 and 3.32, we get 


$$
\left|\Phi_{L}>=G_{L} V_{L S}\right| \Phi_{S}>
$$

and

$$
\left|\Phi_{R}>=G_{R} V_{S R}\right| \Phi_{S}>
$$

where,

$$
G_{L, R}=\frac{1}{E-H_{L, R}}
$$

Equation 3.35 represents the Green's function of left/right electrode. Combining Equations $3.31,3.33$, and 3.34 , we get

$$
\begin{gathered}
V_{L S}^{\dagger} G_{L} V_{L S}\left|\Phi_{S}>+H_{S}\right| \Phi_{S}>+V_{S R}^{\dagger} G_{R} V_{S R}\left|\Phi_{S}>=E\right| \Phi_{S}> \\
\left(E-H_{S}-\Sigma_{L}-\Sigma_{R}\right) \mid \Phi_{S}>=0
\end{gathered}
$$

where $\Sigma_{L}=V_{L S}^{\dagger} G_{L} V_{L S}$ and $\Sigma_{R}=V_{S R}^{\dagger} G_{R} V_{S R}$ are the self energy operators at the left and right interfaces, respectively. The Green's function associated with Equation 3.37 is [24, 25, 87]

$$
G(E)=\frac{1}{E-H_{S}-\Sigma_{L}-\Sigma_{R}}
$$

The Green's function in Equation 3.38 is the main entity in the single particle Green's function approach in determining the ballistic transport in nanoscale junction $[24,25$, 87]. The Green's function in Equation 3.38 is the retarded Green's function, which is 
interpreted, in the time domain, as the response to an impulse excitation at $t=0$ [24]. With this formulation, the complicated problem of entire system is now turned into a problem of active scattering region, which is open to the electrodes through the self energy functions $[24,25]$. The self-energy functions are the non-Hermitian matrices, and the imaginary part of the self-energy matrices gives the broadening or inverse life time of the energy level in the active scattering region. The broadening matrices $\Gamma_{L, R}(E)$ are defined in terms of self-energy matrices as [24, 25]:

$$
\Gamma_{L, R}=i\left[\Sigma_{L, R}-\Sigma_{L, R}^{\dagger}\right]
$$

Finally, the probability of reaching the electron from the source to the drain through active scattering region is determined by the transmission function, which is a summation of transmission probabilities over all possible channels in the active scattering region, and is obtained as $[24,25,87]$ :

$$
T(E)=\operatorname{Tr}\left[\Gamma_{L} G \Gamma_{R} G^{\dagger}\right]
$$

Our next task is to calculate the self energy matrices and the Green's function of a real system under the non-equilibrium situation. To create the non-equilibrium situation, we apply an electric field $(\vec{\varepsilon})$ along the wire axis, and we incorporate this field effect into the Hamiltonian of the active scattering region. The Hamiltonian of the active scattering region with the inclusion of the field becomes [24, 25, 87] 


$$
H(\varepsilon)=H(0)+\vec{\varepsilon} \cdot \sum_{i} \vec{r}(i)
$$

where $H(0)$ is the Hamiltonian of the active scattering region in the absence of field (unperturbed Hamiltonian), and $\vec{r}(i)$ is the coordinate of the $i^{t h}$ electron. The single electron energy levels in the active scattering region are calculated using a finite cluster density functional theory. The use of the real space approach for the active scattering region allows us to extract the Hamiltonian of the molecular part only $\left(H_{m}(E, \varepsilon)\right)$ from the Hamiltonian of active scattering region [87]. The Green's function of the molecular part only then becomes

$$
G_{m}(E)=\left[E \times S-H_{m}-\Sigma_{L}-\Sigma_{R}\right]^{-1}
$$

where $E$ is the injection energy of the incoming electron, $S$ is the overlap matrix whose dimension is equal to the dimension of $H_{m}$, and $\Sigma_{l, r}$ are self energy functions that describe the lead-molecule interactions. The self energy functionals $\left(\Sigma_{L, R}\right)$ can be written in terms of orthogonal-bias-dependent molecule-lead coupling matrices $\left(C_{L, R}\right)$ as $[24,25,87]$ :

$$
\Sigma_{L, R}=C_{L, R}^{\dagger} G_{p} C_{L, R}
$$

Here, $G_{p}$ is the Green's function of the lead, which is considered to be the same for both the left and right leads since similar leads are considered in both sides. In oder to calculate $G_{p}$, in the wide band approximation, first we calculate the bulk density of state (DOS) of a metal from which the lead is formed, and secondly we calculate the DOS per electron in 
the unit cell $(\eta)[87,91]$. Finally, we use the following relation to calculate $G_{p}[87,91]$ :

$$
G_{p}(E)=-i \pi \eta(E) \times I
$$

where $I$ is an identity matrix of dimension $\mathrm{n} \times \mathrm{n}$; $\mathrm{n}$ is the total number of Gaussian basis functions used to represent atoms from the lead in the active region.

Our study includes both the spin polarized and spin unpolarized electron transport through various nano-scale devices. Thus far, I have considered only spin unpolarized transport. Next, I would like to discuss how to calculate the Green's function and self energy matrices for a system which is magnetic in nature.

\subsubsection{Spin-polarized electron transport}

There are two ways we can make the device magnetic: (1) taking a non-magnetic channel and magnetic electrodes, such as Nickel, Cobalt, Iron, etc, and (2) taking a magnetic channel and non-magnetic electrodes. The device having the non-magnetic channel in between two magnetic electrodes is called a spin-valve device [34, 35, 36]. When a ferro-magnetic channel is sandwiched between two non-magnetic electrodes, we term it as a spin filtering device $[52,54]$. First, I would like to discuss a spin-valve device. When both the electrodes are magnetic, there will be two possible magnetic configurations between the left and right electrodes: the first one will be parallel configuration (PC), where 
both the electrodes are magnetized along the same direction, and the second one will be anti-parallel configuration (APC), where two electrodes are magnetized oppositely. When a non-magnetic channel is attached between two magnetic electrodes, it becomes magnetic due to a magnetic proximity effect [92]. As a result, both the molecular Hamiltonian and self energy matrices become magnetic. Thus, the Green's function of the molecular region for a spin-valve devices becomes [92]

$$
G_{m}^{\sigma}(E)=\left[E \times S-H_{m}^{\sigma}-\Sigma_{L}^{\sigma}-\Sigma_{R}^{\sigma}\right]^{-1}
$$

where $\sigma$ represents the spin states $(\uparrow$ or $\downarrow$ ). The spin-polarized self-energy matrices are given by [92]

$$
\Sigma_{L, R}^{\sigma}=C_{L, R}^{\sigma \dagger} G_{p}^{\sigma} C_{L, R}^{\sigma}
$$

The spin-polarized Green's function of the lead is given by

$$
G_{p}^{\sigma}=-i \pi \eta^{\sigma} \times I
$$

In case of PC, $\sigma$ will be same for both the left and right electrodes as [92]:

$$
G_{p L}^{\sigma}=-i \pi \eta^{\sigma} \times I ; G_{p L}^{\sigma}=G_{p R}^{\sigma}
$$

However, in case of APC, Green's functions for left and right leads are different as [92]:

(a) for $\sigma=\uparrow$ 


$$
G_{p R}=-i \pi \eta^{\downarrow} \times I ; G_{p L}=-i \pi \eta^{\uparrow} \times I
$$

(b) for $\sigma=\downarrow$

$$
G_{p R}=-i \pi \eta^{\uparrow} \times I ; G_{p L}=-i \pi \eta^{\downarrow} \times I
$$

Here I have used the notations $G_{p L}$ and $G_{p R}$ to represent the Green's functions of left and right leads, respectively. The way we calculate $\eta^{\sigma}$ for magnetic leads is similar to the non-magnetic leads; however, the DOS of the magnetic system has two components: up spin and down spin states. As a result, $\eta^{\sigma}$ has two components [92]. The next important step is to match the Fermi-energy of active scattering region to the Fermi-energy of bulk electrodes to bring the entire system in equilibrium. Highest Occupied Molecular Orbitals (HOMO) energy is considered as the Fermi energy of the active scattering molecular region for both the PC and the APC.

For the spin-filtering device, the Green's function of the lead $\left(G_{p}\right)$ is spin-unpolarized because we consider the non-magnetic electrodes; however, the self energy matrices and the molecular Hamiltonian are spin-polarized since the channel is made from a ferro-magnetic material [52]. As a result, the Green's function in Equation 3.25 is spin-polarized. The rest of the theory for the spin-filtering device is similar to the spin-valve device. 


\section{Chapter 4}

\section{Charge and Spin Transport in}

\section{Molecular Junctions*}

\subsection{Introduction}

Making an electronic device from a single molecule or a group of molecules is an exciting idea [9], and its successful implement is expected to revolutionize the electronic industry $[10,93,94,95]$. Using molecules to build a device has been pursued for more than four decades; several groups have been successful in demonstrating conduction, rectification,

*Portion of this chapter is copied from the Journal of Physical Chemistry C vol.116, page 17268-17273, year 2012 by K. B. Dhungana, S. Mandal, and R. Pati, and the Applied Physics Letter vol.104, page 162404-162407, year 2014 by K. B. Dhungana and R. Pati. Copyright - Appendix A. DOI: 10.1021/jp3043335 \& DOI: 10.1063/1.4873396 
and switching in molecular junctions; see refs. [6] and [96] for a comprehensive review. However, the experimental difficulty of achieving robust atomic level control at the metal-molecule interface hinders the progress in this field. A statistical approach involving measurement of conductance by repeatedly forming thousands of metal-molecule junctions has been used by researchers to extract reliable data for the conductance in molecular junction $[11,97,98]$. Yet, the qualitative as well as quantitative interpretation of experimental data pose a significant challenge as atomic level structural details of the junctions are not available a priori. In addition, the conformation of the molecule as well as the contact structure, which evolve dynamically during experimental measurements make the theoretical task much harder. Thus, a true understanding of experimental data can only be achieved by a theoretical calculation of conductance in an ensemble of molecular wires with different junction geometries [99, 100, 101, 102].

In addition to charge transport, spin-polarized transport has been studied extensively in organic molecules in recent years; the observed large spin coherence length makes them desirable for spintronics [39]. When a semiconducting molecule acts as a tunnel barrier between two ferromagnetic contacts, the resistance in the circuit depends upon the relative orientation of the magnetization of the contact layers [103, 104, 105]. Usually, the circuit resistance in this device changes from minimum resistance for the parallel spin configuration (PC) to maximum resistance for the antiparallel spin configuration (APC) between the contacts resulting in a spin-valve effect [33] -the foundation behind modern high density data storage device. The relative change in resistance between the $\mathrm{PC}$ and the 
APC in such a device is known as tunnel magnetoresistance (TMR), which is much higher than the magnetoresistance observed in a spin-valve device with a metallic spacer. This has made the TMR device much more appealing as a read head sensor for the high density data storage.

With the advance in nanotechnology, the molecular spintronics has progressed enormously, and it has been possible to build molecular spin-valve devices using different techniques. For example, using a spin-polarized scanning tunneling microscope, researchers have already demonstrated spin valve effect with a tunnel magnetoresistance as high as $60 \%$ in a hydrogen phthalocyanine molecule as a spacer between two ferromagnetic cobalt contacts [43]. In another instance, Bagrets et al. have demonstrated negative TMR of $50 \%$ in the same hydrogen phthalocyanine molecule using a combination of ferromagnetic and antiferromagnetic electrodes [44]. In a pioneering attempt, researchers have recently fabricated a $\mathrm{Ni} / 1,4-$ benzenedithiol/Ni spin-valve junction using the break junction technique $[42,45]$. They reported a very high magnetoresistance as predicted from theory $[48,106,107]$. Until now, only two-terminal, molecular spin-valve (MSV) junctions have received major attention $[41,42,43,44,45,48,92,106,107,108,109]$. Achieving additional control of TMR in such a molecular junction by a gate field is the prerequisite for a spin-valve transistor [50].

This chapter covers both spin-polarized and spin-unpolarized electron transport in molecular junctions and is divided into two sections. In the first section, I will focus on 
charge transport and role of junction configuration in a single molecular device. The effect of gate field on spin-polarized current in a molecular spin-valve transistor is explored in the second section.

\subsection{Junction Dependent Conductance in a Molecular device}

In this section, we investigate the junction dependent current-voltage characteristics in a molecular junction consisting of Ruthenium-bis(terpyridine) interconnect attached between two gold electrodes. The thiolate $(-\mathrm{S})$ group is used to anchor the molecule to the gold (111) electrode; the most likely ONTOP and HOLLOW binding sites are considered. The ONTOP binding site refers to the junction geometry, where the $\mathrm{S}$ atom is directly on the top of the Au atom. For the HOLLOW binding site, the $\mathrm{S}$ atom is on the three fold hollow site of the $\mathrm{Au}(111)$ surface [110]. The Ruthenium-bis(terpyridine) junction is of interest because it has been fabricated and electron transport property has been measured [111] using a scanning tunneling microscope(STM). By sweeping the tip bias from 0 to $2 \mathrm{~V}$, a sudden increase in current is reported at a threshold tip bias $\left(V_{t h}\right)$ of $\sim 1.7 \mathrm{~V}$. The current is reported to be small and remains flat (OFF state) for the bias range below the threshold value. When the tip-bias is increased beyond $V_{t h}$, a current plateau is observed (ON state) [111]. Despite this experimental observation, only nominal theoretical efforts are made to 
identify the origin of such switching behavior in the current. There are several unanswered questions: What causes the current to increase sharply at a threshold bias? Which junction geometry is likely to yield such a non-linear I-V curve? What is the role of conformational change within the molecule? We answer these subtle questions in this article.

We have built numerous prototypical molecular junctions by varying junction geometries, $\mathrm{S}-\mathrm{Au}$ interfacial distance, orientation angle at the junction, and conformational change within the molecule. A single particle Green's function formalism $[24,25,26,99,112$, $113,114,115,116,117,91,118]$ in conjunction with explicit orbital dependent density functional method(DFT) [63] involving B3LYP [119, 120] hybrid functional is used to calculate the electronic current in the molecular junction. Our calculation reveals a sensitive dependence of I-V feature on the junction geometry. For the ONTOP junction geometry, a sharp increase in current after a threshold bias is observed. Conformational change within the molecule is found to have no effect on $V_{t h}$. As we approach the weakly coupled regime by increasing the interfacial distance, the value for $V_{t h}$ is found to decrease and a current plateau is observed for bias beyond $V_{t h}$; the plateau length is found to increase with the increase in interfacial distance $(d)$. A similar feature in I-V is reported by the STM experiment [111]. We look into the bias dependent transmission and orbital characters of the participating eigen-channels to identify the origin of the distinct features observed in the I-V curve.

The rest of this section is organized as follows. In the subsection 4.2.1, we present briefly 
the computational procedure. Result and discussions are described in the section 4.2.2, followed by a brief summary in the section 4.2.3.

\subsubsection{Computational Method}

Our results are based on first principles DFT [63] calculations, which involve Becke's three-parameter hybrid functional (B3LYP) $[119,120]$ for exchange and correlation. The use of explicit orbital dependent B3LYP functional allows us to include a part of the exact Hartree-Fock exchange and thus corrects partly the self-interaction error. This self-interaction corrected scheme $[101,113,118]$ has been reported to yield a lower current value that is found to be in better agreement with the experimentally measured current when compared with the calculated current obtained using other functionals (SVWN, PW91PW91, PW91LYP) [118]. A real-space approach that employs the single determinant wave function is used here. A finite set of Gaussian atomic orbital [119] is used to construct the trial wavefunction. To ensure a tight convergence during the self-consistent calculation, we have used the convergence thresholds for energy, maximum and root mean square electron density as $10^{-6}, 10^{-6}$, and $10^{-8}$ a.u. respectively. The all electron $6-311 \mathrm{G}^{*}$ Gaussian basis set [119] is used to describe all the atoms in the thiolate terminated Ruthenium-bis(terpyridine) complex except the $\mathrm{Ru}$ atom. For the gold atoms in the electrodes and $\mathrm{Ru}$ atom in the molecule, we have used the LANL2DZ pseudo-potential basis set [119] that includes scalar relativistic effect. 
The prototypical two-terminal molecular device is built by sandwiching the Ruthenium-bis(terpyridine) complex between two semi-infinite gold electrodes; thiolate (-S) anchoring groups are used to attach the molecule between the electrodes. The optimized structure for the Ru-terpyridine complex in which the $\mathrm{Ru}$ atom is bonded to two terpyridine molecules in an approximate octahedral geometry is used to build the prototype. This open device has two parts. One part consists of the molecule and a finite number of gold atoms (taken from the Au [111] surface on both sides). This is called the active-scattering region. For the ONTOP configuration, we have included four gold atoms on each side and for the HOLLOW configuration, we have included three gold atoms on each side in the active-scattering region. The other part is the semi-infinite part of the gold electrodes assumed to retain its bulk properties. We have used a single particle bias-dependent Green's function approach $[24,25,26,99,112,113,114,115,116,117,91,118]$. This approach allows us to convert the problem of the complicated experimental device into a problem of the active region, which is open to the electrodes through the self-energy functions $\left(\Sigma_{l, r}\right)$. The bias-dependent Green's function of the device is calculated as [26, 118, 91]:

$$
G(E, \varepsilon)=\left[E \times S-H_{m o l}(\varepsilon)-\Sigma_{l}(\varepsilon)-\Sigma_{r}(\varepsilon)\right]^{-1},
$$

where $H_{m o l}$ is the bias-dependent Kohn-Sham molecular Hamiltonian obtained by suitable partitioning of $H(\varepsilon)$. The use of the real-space approach for the active scattering region allows us to partition the $H(\varepsilon)$ to obtain $H_{m o l}(\varepsilon)$. $E$ is the injection energy of the 
tunneling electron, and $S$ is the molecular overlap matrix. The current is calculated using multi-channel Landuar-Buttiker formula as discussed in Chapter 3, Section 3.3.

\subsubsection{I-V for Different Junction Geometries}

The calculated current-voltage characteristics for different junction geometries are presented in Fig. 4.1. Several intriguing features are noticeable. First, in the case of ONTOP junction geometry at both interfaces (Fig. 4.1a), a sharp increase in current is observed after a $V_{t h}$ for all distances. A closer examination reveals a very small shift in $V_{t h}$ toward a higher value when $d$ changes from 2.42 to 2.62 . It should be noted that 2.42 is the equilibrium S-Au interfacial distance. We have varied the S-Au interfacial distances and compared the total energy for various distances to find the equilibrium configuration. However, as we approach the weakly coupled regime by increasing $d$ further, $V_{t h}$ is found to shift toward a lower value, which suggests that the value for $V_{t h}$ depends non-linearly on the interfacial distance. For higher bias beyond $V_{t h}$, a current plateau (ON state) is observed; the width of the current plateau is found to increase with the increase of $d$. The current remains relatively small and flat (OFF state) prior to $V_{t h}$.

Experimental measurement in a Ruthenium-bis(terpyridine) complex reported [111] a similar I-V feature with current switching at $1.7 \pm 0.25 \mathrm{~V}$. For the interfacial distances considered in our calculation, the value for $V_{t h}$ is found to vary within $\sim 1.5 \mathrm{~V}$ to $\sim 2.0 \mathrm{~V}$. 


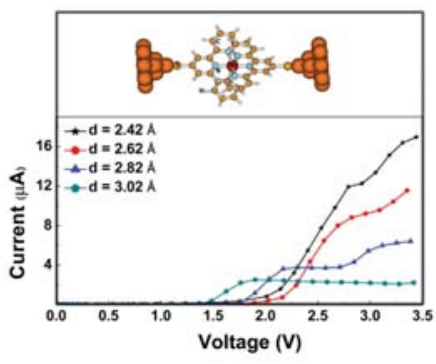

(a) ONTOP-ONTOP

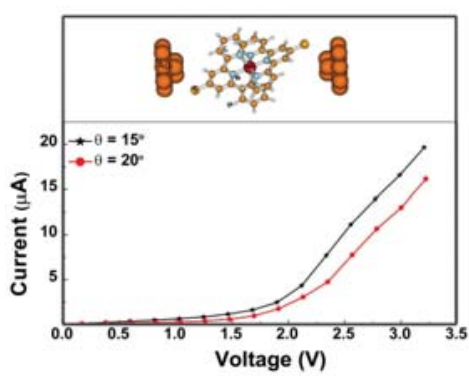

(d) HOLLOW-HOLLOW rotated

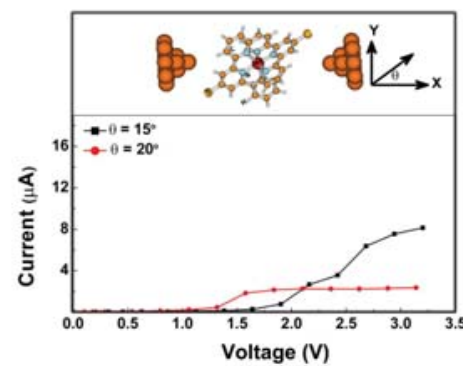

(b) ONTOP-ONTOP rotated

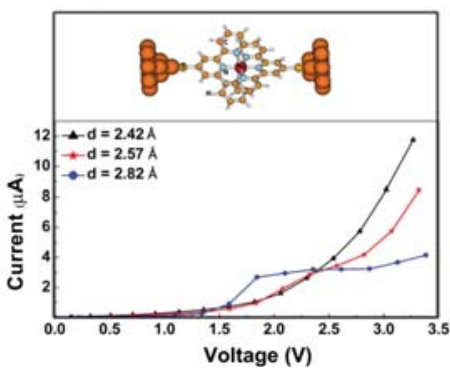

(e) ONTOP-HOLLOW

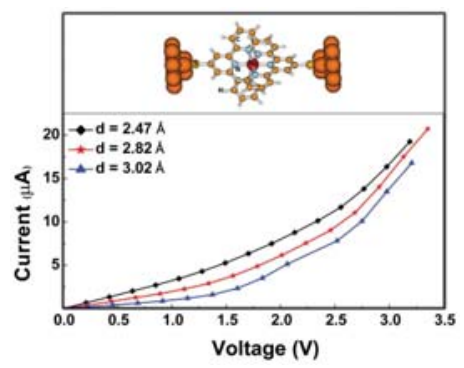

(c) HOLLOW-HOLLOW

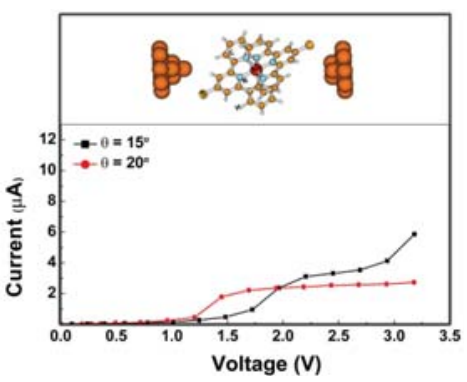

(f) ONTOP-HOLLOW rotated

Figure 4.1: Calculated current(I)-voltage(V) characteristics for Ru-bis(terpyridine) molecular wires with different junction configurations. Top and bottom panels show the junction structure and the corresponding I-V characteristic, respectively. The distance $d$ refers to the inter planar distance between the sulphur and the nearest gold; the $\mathrm{X}$ axis represents the direction of current flow. Reprinted figure with permission from K. B. Dhungana, S. Mandal, and R. Pati, J. Phys. Chem. C 116, 17268-17273 (2012). (c) The American Chemical Society.

For $d=3.02$, our calculation yields a small negative differential resistance (NDR) feature in the current-voltage characteristic. When the molecule is rotated about the Z-axis by $\theta=$ $15^{\circ}$ (Fig. 4.1b), a sharp increase in current is observed after a $V_{t h}$. Increasing the $\theta$ form $15^{\circ}$ to $20^{\circ}$, threshold value shifted from $\sim 1.75 \mathrm{~V}$ to $\sim 1.3 \mathrm{~V}$ and a plateau is observed for higher bias beyond $V_{t h}$ as observed for the weakly coupled case (Fig. 4.1a). 
For the HOLLOW binding sites at both interfaces (Fig. 4.1c), calculated current is found to increase steadily with applied bias for all interfacial distances that we have considered. Unlike the ONTOP junction geometry (Fig. 4.1a), the magnitude of the current for the HOLLOW configuration is found to decrease for higher $d$ over the bias range from 0 to $3 \mathrm{~V}$. Comparing the magnitude of current between junction geometries with ONTOP and HOLLOW binding sites, a higher current is noted for the HOLLOW binding geometry. For example for $d=2.82$, the ONTOP junction geometry yields a current value of $0.024 \mu A$ at $1.35 \mathrm{~V}$ in contrast to $2.910 \mu \mathrm{A}$ for the HOLLOW junction geometry. When the molecule is rotated about the Z-axis (Fig. 4.1d), we do not observe a current plateau in the I-V as found in the weakly coupled junctions with ONTOP binding site; the current is found to increase steadily with the increase in applied bias beyond $V_{t h}$.

In the case of ONTOP junction geometry on one side and HOLLOW on the other (Fig. 4.1e), as we approach the weakly coupled junction by increasing the interfacial distance, a current plateau in I-V beyond $V_{t h}$ is observed. Rotation of the molecule about the Z-axis yields a similar feature in I-V as observed in Fig. 4.1b. These calculations thus confirm that the experimental measurements are most likely for weakly coupled junction with ONTOP junction geometry at one or both $\mathrm{S}$-Au interfaces. It should be noted that though the I-V feature obtained with the ONTOP junction geometry in weakly coupled configuration agrees reasonably well with the experimental data, there is a three orders of magnitude difference in current between theory and experiment; our calculated current value is found to be of the order $\sim \mu A$ as compared to the $\sim n A$ reported from the experiment [111]. The 
discrepancy in the magnitude of current between theory and experiment can be understood as follows. First, the junction considered in the experiment could be much more weakly coupled than the one considered here. Second, our calculation is based on a static exchange correlation potential instead of a true dynamically corrected exchange correlation potential $[121,122,123,124,125]$; the use of static exchange correlation potential is found to overestimates the magnitude of current.

\subsubsection{Conformational Change}

To see if the conformational change in the molecule between the lead causes switching in conductance, we recalculated the current for ONTOP junction geometry $(d=2.42)$ at both interfaces (Fig. 4.2) by rotating one of the terpyridine planes from its equilibrium configuration by an angle $\Phi$. It should be noted that for the equilibrium structure, the two terpyridine planes are almost perpendicular to each other. We found the current remaining relatively flat for bias up to $\sim 2 \mathrm{~V}$ and switches to a higher conductance state for higher bias beyond $\sim 2 V$. A very similar feature in I-V was noted for $d=2.42$ with the ONTOP junction geometry (Fig. 4.1a).

To examine if conformational change considered here is achievable by thermal fluctuation, we calculated the energy difference between extended systems with $\Phi=0^{\circ}$ (equilibrium structure) and $\Phi=10^{\circ}$, which is found to be $0.21 \mathrm{eV}$. This suggests that such a 


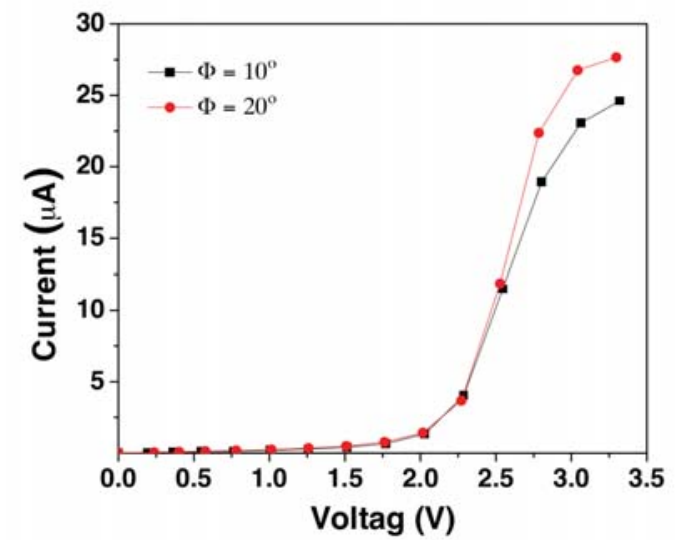

Figure 4.2: Current-Voltage characteristics for Ru-bis(terpyridine) molecular wire with two different conformations of the molecular spacer; $\Phi$ represents the angle of rotation for one terpyridine plane from its equilibrium configuration about the current-flow axis. Reprinted figure with permission from K. B. Dhungana, S. Mandal, and and R. Pati, J. Phys. Chem. C 116, 17268-17273 (2012). (c) The American Chemical Society.

conformational change may not be possible by thermal fluctuation. This study thus rules out that the switching behavior in conductance observed in the experiment is due to conformational change. Comparing the magnitude of current (Fig. 4.1a and Fig. 4.2), we observed a higher magnitude of current after the threshold bias for the molecule with conformational change. Conformational change makes the two terpyridine rings closer to a planar configuration allowing electron to delocalize between the two rings resulting a higher current [125]. 


\subsubsection{Transmission}

To understand the origin of switching behavior of current, we look into the bias dependent transmission function. The chemical potential window (CPW) is shown by the dotted lines and the Fermi energy is set to zero in the energy scale. The area under the transmission curve between the CPW determines the current. Since the I-V feature for the junction with the ONTOP binding site compares reasonably well with the experimental I-V feature, we have presented the bias dependent transmission function for the ONTOP junction geometry. For brevity, we have only plotted bias dependent transmission (Fig. 4.3) for $d=2.42,2.82$ and 3.02 respectively. Several points are noticeable. First, in all cases, the transmission peaks shift toward right with the increase in applied bias. In the case of a strongly coupled junction (Fig. 4.3a), the transmission peaks are outside the chemical potential window (CPW) for $\mathrm{V}=0.985 \mathrm{~V}$ and $2.014 \mathrm{~V}$. This is the reason we do not observe any significant change in current by increasing bias up to $\sim 2 \mathrm{~V}$; the current remains relatively small and flat. Increasing the bias further, for $\mathrm{V}=3.050 \mathrm{~V}$, the chemical potential window encloses transmission peaks at $E=-1.07 \mathrm{eV}$ and $E=1.38 \mathrm{eV}$ resulting in a sudden increase in current. Increasing the bias from $\mathrm{V}=3.050 \mathrm{~V}$ to $3.443 \mathrm{~V}$, the window width for $\mathrm{CPW}$ increases, and an additional transmission peak at $E=1.68 \mathrm{eV}$ appears within the window; this results in a higher current for $\mathrm{V}=3.443 \mathrm{~V}$ than that for $\mathrm{V}=3.050 \mathrm{~V}$.

In case of $d=2.82$ (Fig. 4.3b), for $\mathrm{V}=0.953 \mathrm{~V}$, no transmission peaks appear within the 

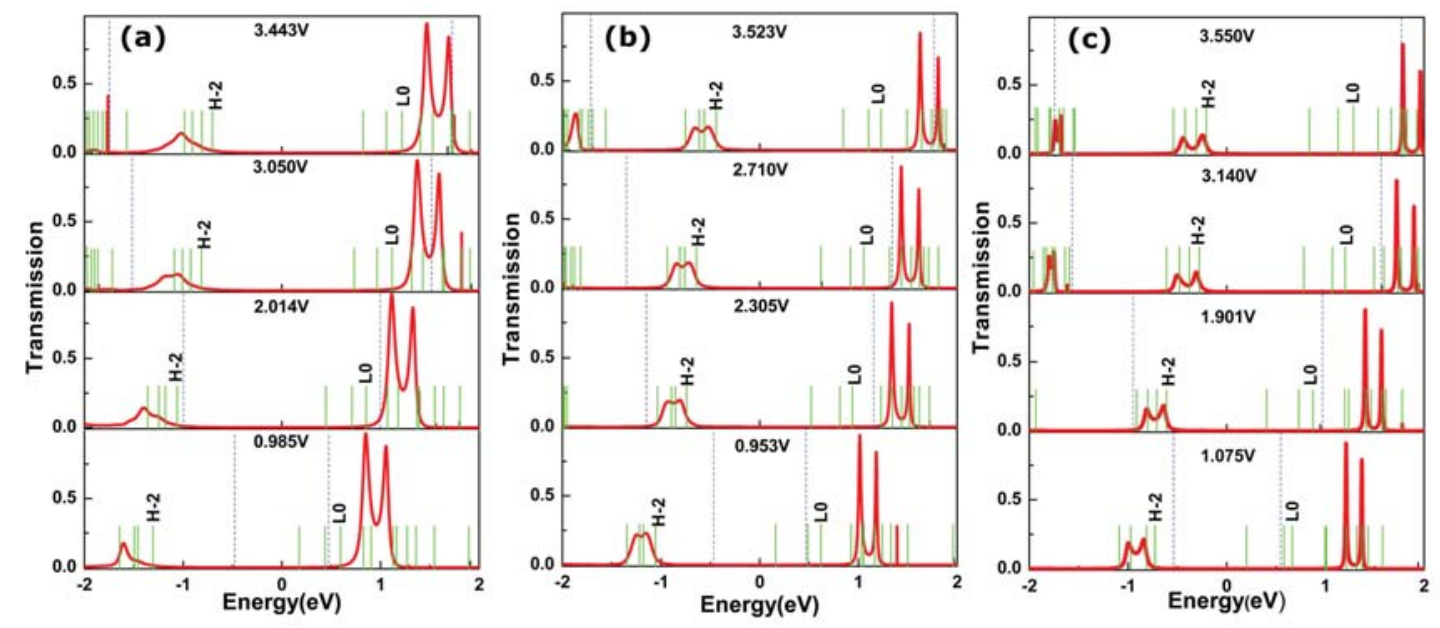

Figure 4.3: Bias dependent transmission as a function of injection energy for interfacial distances of (a) 2.42 , (b) 2.82 , and (c) 3.02 respectively. The Fermi energy is set to zero in the energy scale; the dotted lines in each panel represent the chemical potential window. The eigen channels of the extended molecule are shown by solid vertical lines; L0 and H-2 refer to LUMO and HOMO-2, respectively. Reprinted figure with permission from K. B. Dhungana, S. Mandal, and R. Pati, J. Phys. Chem. C 116, 17268-17273 (2012). (c) The American Chemical Society.

CPW. As we increase the bias to $2.305 \mathrm{~V}$, the transmission peaks at $\sim-0.85 \mathrm{eV}$ appear within the $\mathrm{CPW}$ resulting in a sudden increase in current. Though the $\mathrm{CPW}$ width increases by increasing the bias from $2.305 \mathrm{~V}$ to $2.710 \mathrm{~V}$, no additional transmission peaks appear within the CPW, resulting in a current plateau as shown in Fig. 4.1a. For $\mathrm{V}=3.523 \mathrm{~V}$, the transmission peak at $1.63 \mathrm{eV}$ that appears within the CPW leads to an increase in current. In case of a weakly coupled junction $(d=3.02)$, as expected, the transmission peaks are much sharper. For $\mathrm{V}=1.075 \mathrm{~V}$, no transmission peaks are included within the CPW. As we increase the bias to $1.901 \mathrm{~V}$, the transmission peaks at $\sim-0.65 \mathrm{eV}$ appears within the CPW. No additional peaks appear within the CPW by increasing the bias to $3.140 \mathrm{~V}$. A 

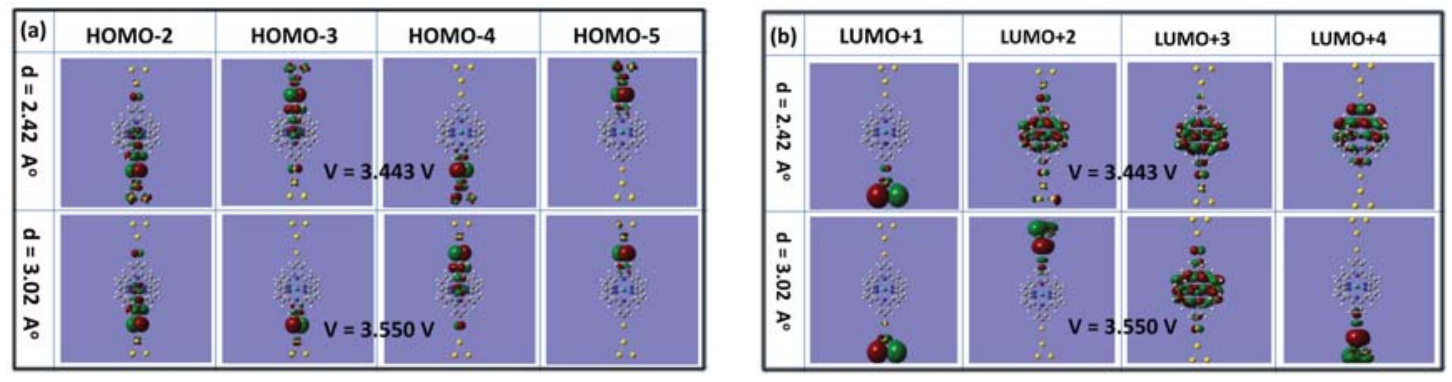

Figure 4.4: Participating bias dependent (a) occupied and (b) unoccupied molecular orbitals for strongly coupled $(d=2.42)$ and weakly coupled $(d=3.02)$ junctions. HOMO and LUMO refer to highest occupied molecular orbital and lowest unoccupied molecular orbital respectively. Reprinted figure with permission from Kamal B. Dhungana, S. Mandal, and and Ranjit Pati, J. Phys. Chem. C 116, 17268-17273 (2012). (c) 2012 American Chemical Society

close look at the height of the transmission peak within the CPW for $\mathrm{V}=1.901 \mathrm{~V}$ and 3.140 $\mathrm{V}$ reveals the transmission peak height to decrease slightly in the case of $\mathrm{V}=3.140 \mathrm{~V}$. This leads to a smaller current for $\mathrm{V}=3.140 \mathrm{~V}$ as compared to $\mathrm{V}=1.901 \mathrm{~V}$ resulting in a slope change in the I-V leading to NDR. A similar observation was noted for the transmission in the Fe-terpyridine molecular junction [26]. A part of the transmission peak at $1.73 \mathrm{eV}$ is included within the $\mathrm{CPW}$ for $\mathrm{V}=3.550 \mathrm{~V}$ resulting in a higher current for $\mathrm{V}=3.55 \mathrm{~V}$. When we compared the transmission for three different $d$, a shift in the transmission peak position toward a higher energy value is observed for a higher $d$.

Further inspection of Fig. 4.3 reveals that the threshold bias in a strongly coupled junction is dictated by the transmission peaks from the unoccupied states. In contrast, for a weakly coupled junction, the threshold bias value is dictated by the transmission peaks from the 
occupied states. For a deeper understanding we analyze the eigen-channels that contribute to the transmission. In the case of a strongly coupled junction $(d=2.42)$, LUMO+1, $\mathrm{LUMO}+2$, and LUMO+3 contribute to the transmission peaks at $0.85 \mathrm{eV}$ and $1.05 \mathrm{eV}$ for $\mathrm{V}=0.985 \mathrm{~V}$. The LUMO+1 has s-orbital character of Au; LUMO+2 and LUMO+3 have $s$-character of gold, $d$-character of $\mathrm{Ru}$ and $p$-character of neighboring $\mathrm{N}$ and $\mathrm{C}$ atoms that connect to Ru. For higher bias $(\mathrm{V}=3.443 \mathrm{~V})$, no significant change in the orbital character is noticed (Fig. 4.4a). HOMO-2, HOMO-3, HOMO-4, and HOMO-5 contribute to the broadened transmission peak at $-1.60 \mathrm{eV}$ for $\mathrm{V}=0.985 \mathrm{~V}$. HOMO-2 and HOMO-3 have $d$-character of $\mathrm{Ru}$ and $d$-character of Au together with the $p$-character of $\mathrm{N}, \mathrm{C}$, and $\mathrm{S}$. HOMO-4 and HOMO-5 have $s$ - and $p$-characters of $\mathrm{C}, p$-character of $\mathrm{S}$, and $d$-character of $\mathrm{Au}$. In the case of a weakly coupled junction, the same set of orbitals contribute to the transmission. The orbital character analysis indicate no contribution from Au for HOMO-2, HOMO-3, HOMO-4, and HOMO-5 (Fig. 4.4a); in the weakly coupled junction, LUMO+1 and LUMO+2 have $s$-character for Au and S, LUMO+3 has almost no contribution from $\mathrm{Au}$ (Fig. 4.4b). The contribution from $s$-orbital of Au to the transmission leads to a sudden increase of current in a strongly coupled junction than that in the weakly coupled junction. Thus, this orbital analysis provides an explanation for the observed higher current after the threshold bias in a strongly coupled junction as compared to the weakly coupled junction; the much higher current in the strongly coupled junction is due to metal $(\mathrm{Au})$ dominated states. 


\subsubsection{Conclusions}

We present first principles calculation of current-voltage characteristics in a Ru-bis(terpyridine) molecular junction, which has been fabricated and characterized using STM. An ensemble of prototypical device structures is constructed by varying metal-molecule binding site, interfacial distance, orientation of the molecule at the interface, and conformational change in the molecule to study junction dependent conductance. A bias dependent Green's function approach in conjunction with an orbital dependent DFT is used to study the I-V characteristics. Comparison of calculated I-V with the experimental data allows us to identify the possible atomic level structural details of the metal-molecule interface in the experimental measurement; the weakly coupled ONTOP junction geometry is found to yield I-V curve with a switching feature that agrees reasonably well with the experimental result. Analysis of bias dependent transmission and orbital character of the participating eigen-channel is presented to explain the origin of distinct I-V features observed in strongly and weakly coupled junction geometries. In the weakly coupled junction, occupied orbitals having $d$-character of Ru and $p$-characters of $\mathrm{S}$, $\mathrm{C}$ and $\mathrm{N}$ in the molecular interconnect are found to dictate the switching behavior in I-V. In contrast, in the strongly coupled junction, the unoccupied gold dominated states dictate the sudden increase of current at a threshold bias. Thus, this study provides an electronic structure level explanation of the observed switching behavior in the Ru-bis(terpyridine) molecular junction. 


\subsection{Molecular Spin-Valve Transistor}

In this section, we have studied the effect of gate field on spin-polarized currents in a molecular spin-valve transistor, where Ru-bis-terpyridine (RBT) molecule is used as an interconnect between two ferromagnetic nickel contacts. Our first-principles quantum transport calculations show a bias dependent variation in TMR with a peak value of 350\% at $0.8 \mathrm{~V}$ in the absence of gate field. A modest change in the gate field from $0 \mathrm{~V} / \AA$ to $0.4 \mathrm{~V} / \AA$, which is experimentally accessible, leads to a significant amplification in the peak value of TMR to $1470 \%$ at $0.3 \mathrm{~V}$. We have unambiguously identified the root cause for such amplification, which is attributed to the gate field induced increase in majority spin current in the PC at low bias arising from the shift in participating frontier molecular orbitals levels towards the Fermi-energy; minority spin current does not exhibit appreciable change with gate field in the same bias range. In the case of APC, both majority and minority spin currents are not affected by gate field at low bias. Orbital analysis indicates that the hybridized orbitals at the interface play a key role in determining the spin dependent current in the device. 


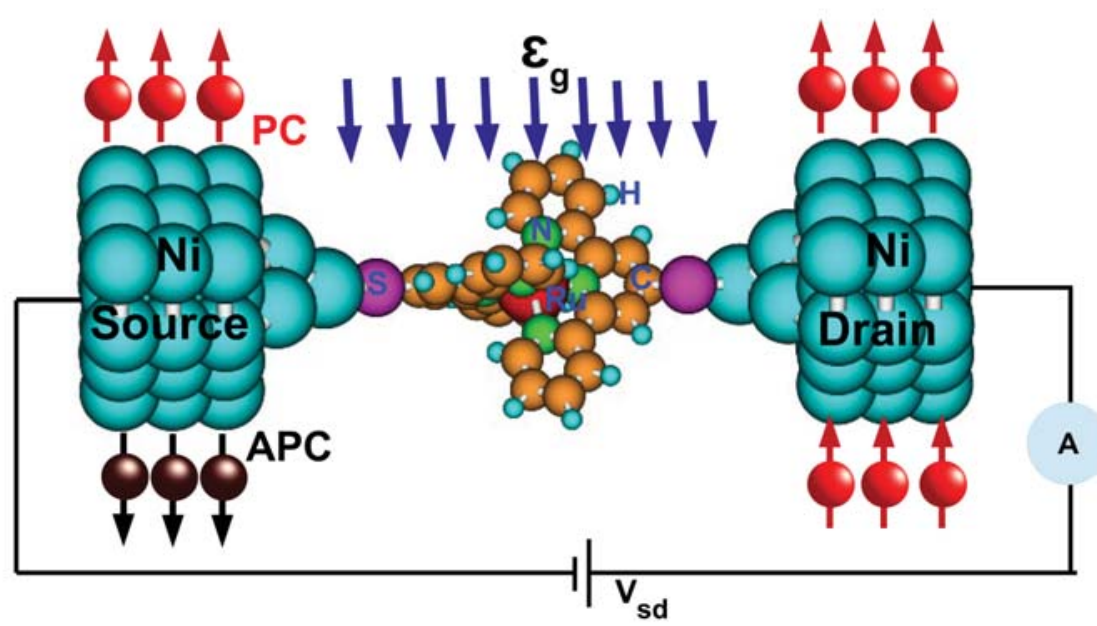

Figure 4.5: Schematic diagram of a molecular spin-valve transistor. Reprinted figure with permission from K. B. Dhungana and R. Pati, Appl. Phys. Lett. 104, 162404-162407 (2014). (c) 2014 AIP Publishing LLC.

\subsubsection{Modeling of a Spin-Valve Transistor}

The molecular spin valve device that we have investigated is shown in Fig. 4.5. The thiolate $(-S)$ anchoring groups are used to attach the RBT molecule between two ferromagnetic nickel (111) electrodes. The choice of RBT molecule is prompted by experimental measurement of charge transport in the RBT molecular junction [111]. The molecular geometry is fully optimized within density functional theory that uses a posteriori B3LYP hybrid exchange-correlation functional [120]. The LANL2DZ effective core potential basis set [126], which includes scalar relativistic effects, is used to describe the heavy atoms such as Ruthenium and Nickel; a triple valance zeta Gaussian basis function (6-311G*) is used for the rest of the atoms in the device. A tight convergence criterion $\left(10^{-8}\right.$ a.u. 
for energy, $10^{-6}$ a. u. for both maximum and root-mean square electron density) with ultra fine grid for numerical integration is used during our self consistent calculations. The interfacial distance between sulfur and the nearest nickel surface of the lead is $2.2 \AA$, which is obtained by minimizing the repulsive interaction within the spin unrestricted density functional theory [63]. This real space approach allows us to construct the retarded spin polarized Green's function $\left(G^{\sigma}\right)$ of the open device $[24,25,47,127]$ by dividing it into two parts: (a) the active scattering region that consists of the molecular complex together with a finite number of nickel atoms from the lead giving the Hamiltonian matrix of dimension $958 \times 958$ for each spin component, and (b) the rest of the electrode on each side that is assumed to retain its bulk (3D) property; 3D leads have been found [47] to better represent the experimental features. The $G^{\sigma}$ is evaluated self-consistently for each applied bias point. To mimic the gate field, we have included a dipole interaction term $\left(\vec{\varepsilon}_{g} \cdot \sum_{i} \vec{r}(i)\right)$ to the core Hamiltonian during self-consistent electronic structure calculation [118]. This permits us to include both first and higher order Stark effects. Then we recourse to a coherent spin conserved tunneling approach developed in Ref. 92 to calculate the spin dependent current of the device.

\subsubsection{Spin Polarized currents}

The results for bias dependent spin polarized current as a function of gate field for PC and APC are presented in Fig. 4.6; the APC is found to be energetically more stable $(\sim 0.03 \mathrm{eV}$ 

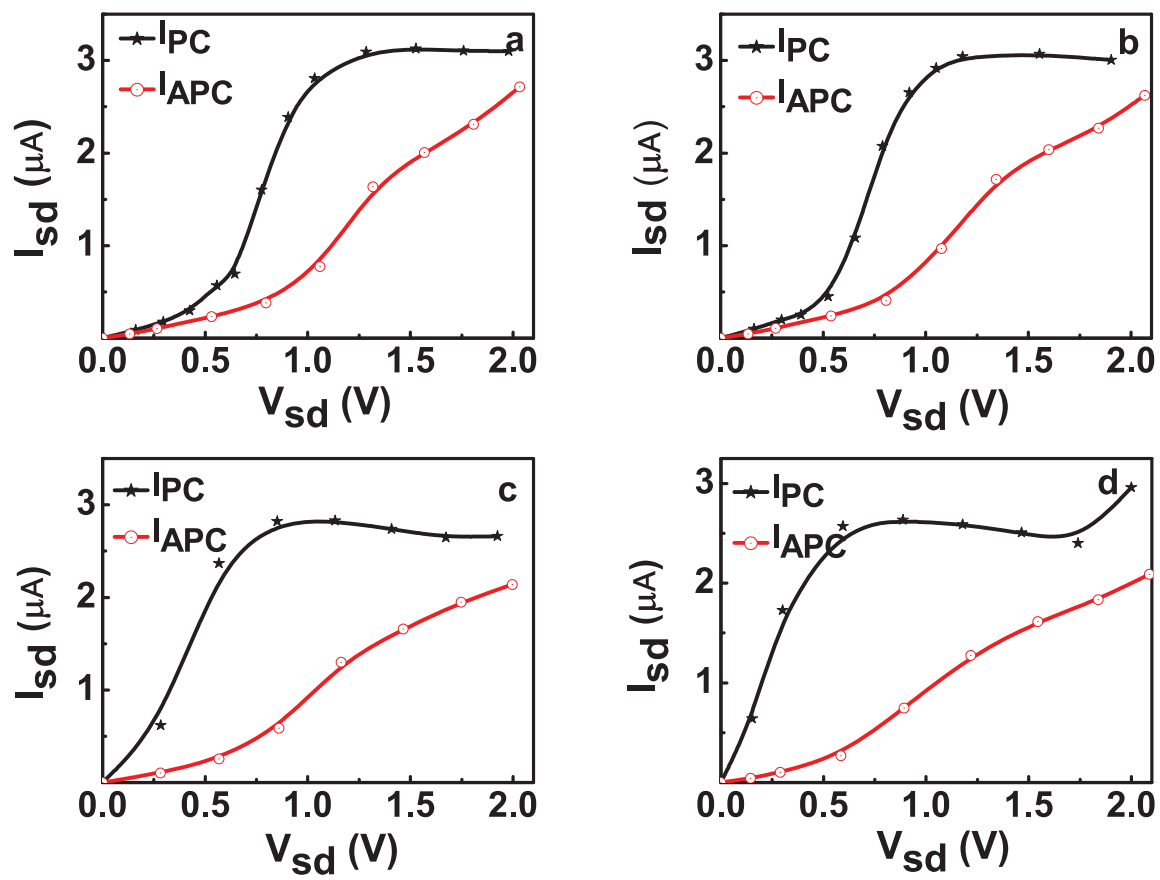

Figure 4.6: $\operatorname{Current}\left(I_{s d}\right)$-Voltage $\left(V_{s d}\right)$ characteristics in a Ru-bis-terpyridine molecular tunnel junction for parallel spin configuration (PC) and anti-parallel spin configuration ( APC) at different gate fields: (a) $\varepsilon_{g}=0.0 \mathrm{~V} / \AA$, (b) $\varepsilon_{g}=0.1 \mathrm{~V} / \AA$, (c) $\varepsilon_{g}=0.3 \mathrm{~V} / \AA$, (d) $\varepsilon_{g}=$ 0.4 V/Å. Reprinted figure with permission from K. B. Dhungana and R. Pati, Appl. Phys. Lett. 104, 162404-162407 (2014). (c) 2014 AIP Publishing LLC.

in the absence of gate field) than the PC. Several interesting features are noticeable. First, irrespective of the applied $\varepsilon_{g}$, as in a typical spin-valve device, the current for the PC $\left(I_{P C}\right)$ is found to be higher than the current for the APC $\left(I_{A P C}\right)$. In the absence of $\varepsilon_{g}$ (Fig. 4.6a), $I_{P C}$ increases steadily for a bias up to $\sim 0.5 \mathrm{~V}$ beyond which it promptly transitions to a higher conductance state; however, the transition in $I_{A P C}$ is much more gradual and occurs at a higher bias $(\sim 1.0 \mathrm{~V})$. We term the bias value where $I_{P C}$ suddenly changes to a higher value as the threshold voltage $\left(V_{t h}\right)$. As we increase $\varepsilon_{g}$ (Fig. 4.6), we find the $V_{t h}$ gets closer 
to $0 \mathrm{~V}$. Unlike the $I_{P C}$, the $I_{A P C}$ does not change with $\varepsilon_{g}$ at a small bias. But at a higher bias $(>1.0 \mathrm{~V})$, both the $I_{P C}$ and $I_{A P C}$ decrease steadily with the increase in $\varepsilon_{g}$. Interestingly, $I_{P C}$ shows a negative differential resistance (NDR) behavior at a higher bias, which is much more prominent for $\varepsilon_{g}=0.4 \mathrm{~V} / \AA($ Fig. $4.6 \mathrm{~d})$. To understand the distinct features of $I_{P C}$ and $I_{A P C}$ in Fig. 4.6, we analyze the $\alpha$ and $\beta$ components of the total spin currents $\left(I_{\alpha}\right.$ and $\left.I_{\beta}\right)$ for PC and APC. In the case of PC, the major contribution to the total current comes from the $\alpha$ states. For APC, as expected, both $I_{\alpha}$ and $I_{\beta}$ are almost same for bias up to $\sim 0.25 \mathrm{~V}$, beyond which $\alpha$ current dominates the $\beta$ current. To quantify this spin-selective resistive property of the device, we examine the spin-injection coefficient, $\eta=\left(I_{\alpha}-I_{\beta}\right) /\left(I_{\alpha}+I_{\beta}\right)$, for PC and APC at a small bias $(0.1 \mathrm{~V})$ with different gate field. In the case of APC, $\eta=0$ at a small bias as $I_{\alpha}=I_{\beta}$. For PC, $\eta$ increases steadily with $\varepsilon_{g}$ as shown in Fig. 4.3a; a very high spin injection efficiency of $98 \%$ is achieved at low bias with $\varepsilon_{g}=0.4 \mathrm{~V} / \AA$.

Next, we turn our focus to TMR, which is calculated using the common optimistic definition $[48,106]: \operatorname{TMR}=\left(I_{P C}-I_{A P C}\right) / I_{A P C}$. Fig. $4.7 \mathrm{~b}$ summarizes the bias and $\varepsilon_{g}$ dependent TMR values. In the absence of $\varepsilon_{g}$, TMR increases from $60 \%$ at $0.2 \mathrm{~V}$ to reach a peak value of $350 \%$ at $0.8 \mathrm{~V}$ and then gradually drops to $20 \%$ at $2.0 \mathrm{~V}$. Since achieving atomic level control at the molecule-lead interface is a daunting challenge from the experimental perspective, we have varied the interfacial distance between the molecule and lead to gauge the junction dependent effect on TMR. For the two representative interfacial distances (1.9 $\AA$ and $2.5 \AA$ ), a similar bias dependent variation of TMR is noted. When we apply the gate field, the variation of TMR with $V_{s d}$ follows a similar trend, but 

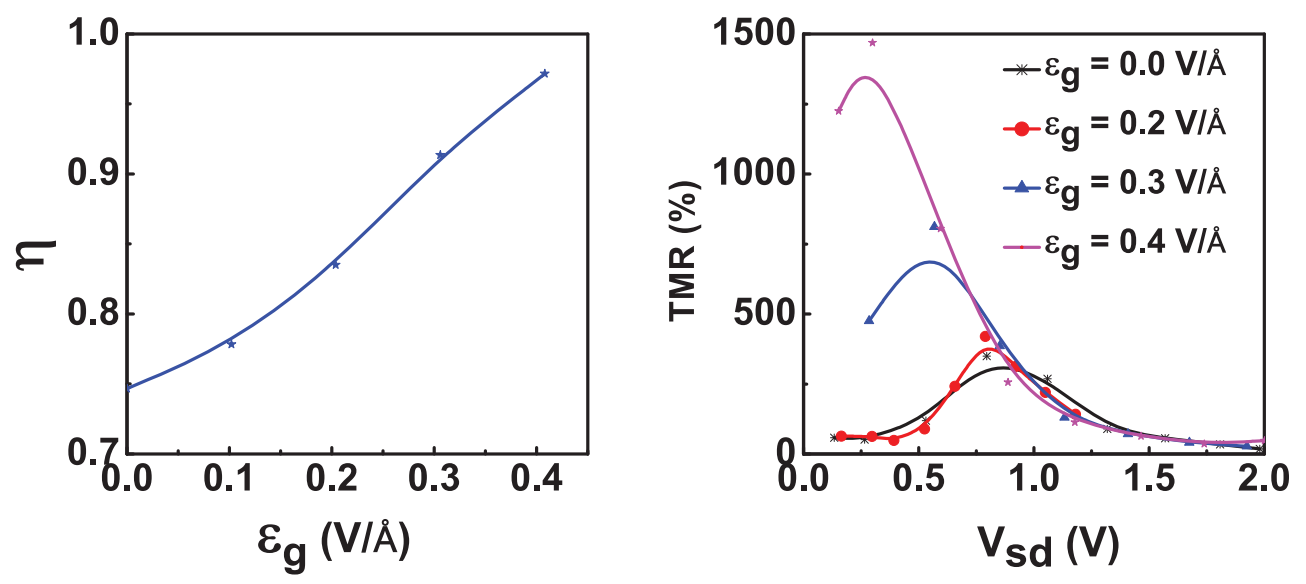

Figure 4.7: (a) Spin-injection coefficient $(\eta)$ for the parallel spin configuration as a function of gate field $\left(\varepsilon_{g}\right) ; \eta$ is zero for the antiparallel spin configuration at low bias. (b) Bias $\left(V_{s d}\right)$ dependent TMR as a function of gate field. Reprinted figure with permission from K. B. Dhungana and R. Pati, Appl. Phys. Lett. 104, 162404-162407 (2014). (c) 2014 AIP Publishing LLC.

the magnitude of the peak value of TMR enhances significantly. For example, when we change the gate field from $0.0 \mathrm{~V} / \AA$ to $0.4 \mathrm{~V} / \AA$, the peak value of TMR increases from $350 \%$ to $1470 \%$ (320\% amplification), and the position of the peak shifts from $0.8 \mathrm{~V}$ to $0.3 \mathrm{~V}$.

\subsubsection{Transmission}

In order to gain a deeper understanding of the observed giant amplification in TMR, we examine the spin polarized transmission for PC and APC. First, we focus on the low bias transmission. In the case of PC (Fig. 4.8a), for $\varepsilon_{g}=0 \mathrm{~V} / \AA$, the transmission peaks from 

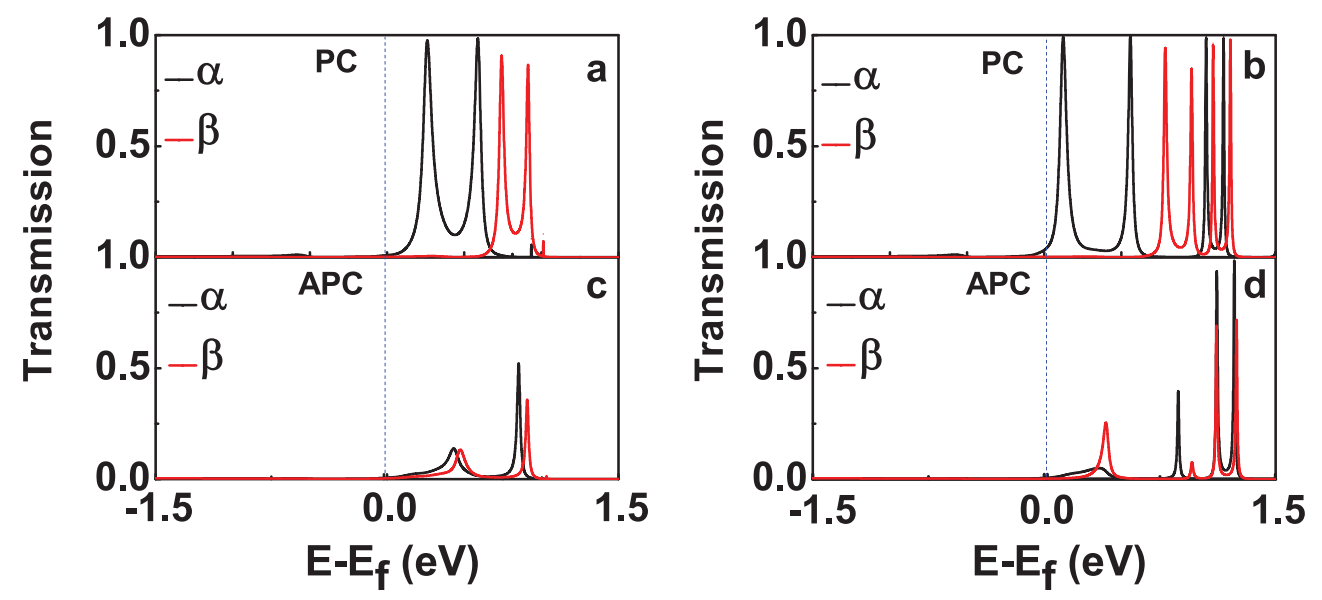

Figure 4.8: Gate field dependent spin polarized transmission at $V_{s d} \sim 0.3 \mathrm{~V}$ for the parallel (PC) and the anti-parallel (APC) spin configuration. (a) $\varepsilon_{g}=0.0 \mathrm{~V} / \AA$, (b) $\varepsilon_{g}=0.4 \mathrm{~V} / \AA$, (c) $\varepsilon_{g}=0.0 \mathrm{~V} / \AA$, (d) $\varepsilon_{g}=0.4 \mathrm{~V} / \AA$. Notation: $\alpha$ stands for spin up and $\beta$ stands for spin down states. Reprinted figure with permission from K. B. Dhungana and R. Pati, Appl. Phys. Lett. 104, 162404-162407 (2014). (c) 2014 AIP Publishing LLC.

$\alpha$ states are closer to the Fermi energy than the beta states resulting in a higher current from the $\alpha$ states than the $\beta$ states. As we increase the gate field from $0 \mathrm{~V} / \AA$ to $0.4 \mathrm{~V} / \AA$ (Fig. 4.8b), the transmission peak position for the $\alpha$ state at $0.26 \mathrm{eV}$ is shifted to 0.12 $\mathrm{eV}$ bringing it much closer to the Fermi energy. In contrast, the $\beta$ states do not show a strong response to $\varepsilon_{g}$. This explains why we see a much higher current from the $\alpha$ state than the $\beta$ state with the increase of $\varepsilon_{g}$, which is also reflected from the $\varepsilon_{g}$ dependent $\eta$ values (Fig. 4.7a). In the case of APC, at zero bias, both $\alpha$ and $\beta$ states transmissions are identical as expected. A small discrepancy between $\alpha$ and $\beta$ states in Fig. 4.8c is due to small bias induced asymmetry. When we increase the $\varepsilon_{g}$ (Fig. $4.8 \mathrm{~d}$ ), though height of the transmission peak (at $0.48 \mathrm{eV}$ ) from the $\beta$ state increases and the transmission peak height 
at $0.41 \mathrm{eV}$ from the $\alpha$ state decreases, no change in transmission values are found within the close vicinity of the Fermi-energy. This leads to no appreciable change in APC current at a low bias (Fig. 4.6). For higher bias $(\sim 1.8 \mathrm{~V})$, at $\varepsilon_{g}=0.4 \mathrm{~V} / \AA$, the transmission peaks position for PC and APC (Figs. 4.9a and 4.9b) move away from the Fermi energy for both $\alpha$ and $\beta$ states. A closer inspection of Fig. 4.9a reveals that the height of the transmission peaks for both $\alpha$ and $\beta$ states in PC decreases at $V_{s d}=\sim 1.8 \mathrm{~V}$ resulting in a NDR feature in current. However, in the case of APC (at $V_{s d}=\sim 1.8 \mathrm{~V}$ ), the transmission peak from $\alpha$ state increases; $\beta$ states are not affected. This leads to an increase in current for the APC with the increase of $V_{s d}$.
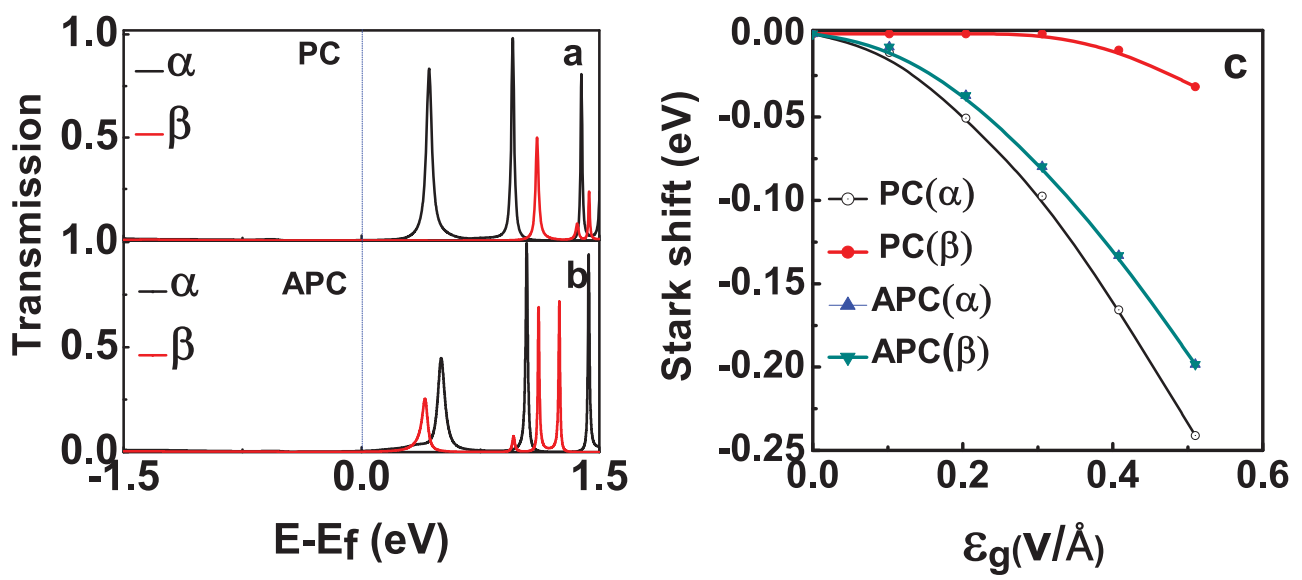

Figure 4.9: Transmission plots for (a) PC and (b) APC at bias $\sim 1.8 \mathrm{~V}$ and $\varepsilon_{g}=0.4 \mathrm{~V} / \AA$. (c) Gate field dependent Stark shift associated with the Lowest Unoccupied Molecular orbital (LUMO) for PC and APC. Notation: $\alpha$ stands for spin up and $\beta$ stands for spin down states. Reprinted figure with permission from K. B. Dhungana and R. Pati, Appl. Phys. Lett. 104, 162404-162407 (2014). (C) 2014 AIP Publishing LLC.

Since the unoccupied frontier molecular orbitals (MOs) are found to provide the spatial 
path for transmission of spin polarized electrons from source to drain in all cases (Figs. 4.4 and 4.5), we analyze the lowest unoccupied molecular orbital (LUMO) for $\alpha$ and $\beta$ states in $\mathrm{PC}$ and $\mathrm{APC}$ for the active scattering region. In the case of $\mathrm{PC}$, for the $\alpha$ state, at $\varepsilon_{g}=$ $0.0 \mathrm{~V} / \AA$, in addition to the ruthenium d-state at the octahedral position, which mediates the coupling between the two perpendicular ter-pyridine complexes, the nickel d, s and sulfur $\mathrm{p}$ states contribute to the LUMO at a low bias; in contrast, for the $\beta$ state, the LUMO has a very small $\mathrm{d}$ and s components of nickel lead.This explains why we see a much higher transmission from the $\alpha$ state near the Fermi energy (Fig. 4.8a). In the case of APC, for both $\alpha$ and $\beta$ states, nickel $\mathrm{d}$ as well as s, and sulfur $\mathrm{p}$ states at one interface only contribute to the LUMO, resulting in a smaller transmission (Fig. 4.8c) near the Fermi energy. When we increase $\varepsilon_{g}$ from $0.0 \mathrm{~V} / \AA$ to $0.4 \mathrm{~V} / \AA$, in the case of PC, the electron distribution in the molecule for the $\alpha$ state localizes along the direction of gate field resulting in a shift in energy level toward the Fermi energy. For $\beta$ states, the response to $\varepsilon_{g}$ is found to be much weaker. In the case of APC, for $\varepsilon_{g}=0.4 \mathrm{~V} / \AA$ (Fig. $\left.4.8 \mathrm{~d}\right)$, we find the electron distribution for both $\alpha$ and $\beta$ states to localize in the direction of $\varepsilon_{g}$. Increasing the $V_{s d}$ for PC (Figs. 4.9a) leads to localization of electron distribution along the direction of current carrying axis causing the transmission peak heights to decrease. However, in the case of APC, interface states (p-component of sulfur and small $d$-component of nickel) contribute to the LUMO for the $\alpha$ state with the increase of bias. To quantify the orbitals' response to the gate field, we examine the Stark shift $\left(\varepsilon\left(\varepsilon_{g}\right)-\varepsilon\left(\varepsilon_{g}=0\right)\right)$ corresponding to $\alpha$ and $\beta$-LUMO for PC and APC (Fig. 4.9c). Fig. 4.9c shows that $\alpha$ state exhibits much stronger Stark shift with 
increase in gate field in comparison to $\beta$ state for PC confirming the distinct spin dependent transmission; for APC, both the $\alpha$ and $\beta$ states show same Stark shift as expected.

\subsubsection{Conclusions}

In conclusion, we have demonstrated a molecular spin valve transistor by showing giant amplification of tunneling magnetoresistance in a single molecular junction via gate field. Our first-principles quantum transport calculations reveal that a modest change in the gate field from 0 to $0.4 \mathrm{~V} / \AA ̊ \AA$, which is experimentally accessible [15], can lead to a $320 \%$ change in TMR. Despite the challenges, the recent experimental demonstration of single molecular transistors $[15,16]$ suggest that our prediction of a molecular spin valve transistor would open up experimental initiative toward its practical realization. 


\section{Chapter 5}

\section{Electrical tuning of spin-current in a}

\section{boron nitride nanotube quantum dot*}

\subsection{Introduction}

Spintronics, which relies on the spin state of the electron to store, transport, and process information, has been the subject of intense research since the discovery of giant magneto-resistance [33]. With the revolutionary progress in nanotechnology in recent years enabling the manipulation of electron spins in nanoscale tunnel junctions $[43,45,128,129,130,131,132,133]$, it has crossed the boundary of conventional, all

\footnotetext{
*Portion of this chapter is copied from the Physical Chemistry Chemical Physics vol. 16, page 7996-8002,
} year 2014 by Kamal B. Dhungana and Ranjit Pati. Copyright - Appendix B. DOI: 10.1039/C4CP00325J 
metallic, solid state multi-layered structures $[35,34,134]$ to reach a new frontier, where nano-structures are being used as controlled spin-carriers. When a quantum-confined nanostructure (QCNS) having a non-magnetic character is used as a tunnel barrier between two magnetic electrodes, it offers new opportunities for the spin manipulation via external electric field — an important prerequisite for nanoscale spintronics $[39,135,136]$; the QCNS in contact with a ferromagnetic lead loses its non-magnetic property due to the magnetic proximity effect and becomes spin-polarized [137, 138]. The external electric field then not only modulates the shape of the spin orbital [136] and position of the discrete spin-polarized eigen-channels of the QCNS due to the Stark effect, but also it modifies the electronic and magnetic structure at the interface $[39,47,132,135,139,140]$, which plays a dominant role in dictating the spin current behavior of the device.

For example, using molecular quantum dot, researchers have already demonstrated giant magnetoresistance effect in molecular tunnel (MT) devices [43, 45, 133]. However, the strong sensitivity of magnetoresistance to the junction structure [92] and the difficulty in achieving atomic level controls at the interface make the implementation of the MT device an arduous task. Other promising nano-structures being investigated as spin-carriers are carbon nanotube quantum dots (CNTQD) coupled to ferromagnetic electrodes $[40,129$, 130]. The gate field induced switching of the exchange field has already been established in this system [129]. In addition, the sign modulations of tunnel magnetoresistance (TMR) in both two and three terminal CNTQD-magnetic junctions have been reported $[130,141]$. But the difficulty in separating the metallic CNT from the semiconducting one poses a 
significant hurdle toward their practical applications in spintronics. On the other hand, a robust semiconducting boron nitride nanotube $(\mathrm{BNT})[142,143]$, which is structurally similar to CNT, would be an ideal tunnel barrier for the spin transport because its electronic property is independent of its chirality. BNT is also found to exhibit a giant response to the transverse electric field [144] due to strong Stark effect arising from the ionic nature of BN bonds - offering new opportunities to control spin current via electric field. However, up until now, no efforts have been made to understand the spin current in such a device.

Here, we use a boron nitride nanotube quantum dot (BNTQD) as a tunnel barrier between two ferromagnetic nickel electrodes to probe the electric field manipulation of spin current. Our first-principles investigation reveals transverse electric field $\left(\varepsilon_{g}\right)$ induced switching in the sign of exchange coupling $(\mathbf{J})$ and tunnel magneto resistance together with a very high spin injection efficiency. The precise role of BNTQD/Ni interface on switching the sign of $\mathbf{J}$ and TMR is identified. In addition, we have observed an intriguing bias dependent switching in spin-polarized current with a robust negative differential resistance (NDR) feature at a higher $\varepsilon_{g}$. The origin of this novel switching property is attributed to the strong field-induced modification of the spin orbitals due to the Stark effect.

The rest of the chapter is organized as follows. In the section 5.2, we present briefly the device modeling. Results and discussions are described in the section 5.3, followed by a brief conclusion in the section 5.4. 


\subsection{Device modeling}

The spin-up and the spin-down components in the presence of $\varepsilon_{g}$ are calculated within the multichannel Landauer-Büttiker formalism: $I_{s d}^{\sigma}=\frac{e}{h} \int_{\mu_{1}}^{\mu_{2}} T^{\sigma}\left(E, V_{s d}, \varepsilon_{g}\right) \times\left[f\left(E, \mu_{2}\right)-\right.$ $\left.f\left(E, \mu_{1}\right)\right] \times d E . \quad T^{\sigma}\left(E, V_{s d}, \varepsilon_{g}\right)$ is the transmission function obtained from the bias dependent spin-polarized Green's function, which is calculated as: $G^{\sigma}\left(E, V_{s d}, \varepsilon_{g}\right)=[E \times$ $\left.S_{Q D}-H_{Q D}^{\sigma}\left(V_{s d}, \varepsilon_{g}\right)-\Sigma_{l}^{\sigma}\left(V_{s d}, \varepsilon_{g}\right)-\Sigma_{r}^{\sigma}\left(V_{s d}, \varepsilon_{g}\right)\right]^{-1} \cdot \mu_{1,2}$ are the electro-chemical potentials at the leads, which are determined self-consistently (see Ref. 92 for details). $H_{Q D}\left(V_{s d}, \varepsilon_{g}\right)$ is the bias dependent Kohn-Sham Hamiltonian for the BNTQD; $\Sigma_{l, r}^{\sigma}\left(V_{s d}, \varepsilon_{g}\right)$ are the bias dependent spin-polarized self-energy functions, which permit the BNTQD to exchange its spin-polarized electrons and energy with the semi-infinite electrodes. We have considered a chemically bonded junction where the ground state based DFT has been found to be a good approximation $[28,112,115,116,117,145]$. The interfacial distance between BNTQD and nickel surface is $1.9 \AA$, which is obtained by minimizing the repulsive interaction within the spin unrestricted density functional theory. The energy versus interface distance curve is found to be parabolic around $1.9 \AA$ that further justifies the use of ground state based DFT in our calculations. An orbital dependent B3LYP hybrid functional for exchange-correlation and an all-electron 6-311g* Gaussian basis set [126] is used to describe the atoms in the device. A true dynamically corrected spin-polarized exchange correlation potential $[123,121]$ would better represent the transport properties; however, it is difficult to implement in such a system. It should be noted that the use of all electron 
basis set leads to a spin-polarized Hamiltonian matrix of the active scattering region with a dimension of $1572 \times 1572$ for each applied bias point. An energy grid of $0.001 \mathrm{eV}$ is used for integration of the transmission. The real space approach adopted here allows us to include the most crucial electronic and magnetic structure details of the BNTQD junction from the first-principles. The modification in the electronic and magnetic structure of the device due to the transverse electric field $\left(\varepsilon_{g}\right)$ is incorporated through the inclusion of a dipole interaction term $\left(\vec{\varepsilon}_{g} \cdot \sum_{i} \vec{r}(i)\right)$ in the Kohn-Sham Hamiltonian as a perturbation; the strength of the dipole interaction is much weaker compared to the electronic interaction. Since the perturbed dipole interaction term contains only single particle interactions, we add it to the core Hamiltonian during the self-consistent electronic structure calculation to include both the first and higher order Stark effects.

\subsection{Spin Polarized Transport}

First, we consider a prototypical BNTQD-magnetic tunnel junction as shown in Fig. 5.1. For a practical reason, an optimized $(6,0)$ BNT of length $12.3 \AA$ is sandwiched between two $\mathrm{Ni}$ (111) electrodes to build the open device structure. It should be noted that tunnel junction with CNTQD channel of diameter of $\sim 1 \mathrm{~nm}$ has been fabricated [131]. Furthermore, gate field induced amplification in a molecular transistor with channel length as small as ours' $(\sim 1 \mathrm{~nm})$ has been demonstrated [16]. Since the electrons in the BNT considered here are strongly confined in all three dimensions, and there is a lattice 
mismatch between BNT and Ni at the interface, we term it as a BNTQD. Then, we recourse to a bias dependent, single particle many-body Green's function approach [24, 25, 47, 127] to obtain the spin dependent current in the BNTQD tunnel device for the parallel spin configuration (PC) and the anti-parallel spin configuration (APC) between the electrodes. Unlike our early work [26], here the bias effect is included self-consistently [92]. It is important to note that the self-consistent inclusion of bias allows us to create an imbalance in carrier concentration at the leads; on one lead there is a charge surplus and at the other lead there is a charge depletion resulting to residual resistivity dipoles [25]. This is reflected in the bias dependent planar average electrostatic potential profiles for the PC and APC (Fig. 5.2). Both the profiles show an almost linear drop in the potential across the junction with constant potentials at the leads. The magnitude of the potential drops at two leads for both PC and APC are different, confirming the asymmetric nature of the BNTQD-Ni interfaces at the electronic level.

Spin polarized current. Since the spin coherence length is expected to be longer than the length of the BNTQD channel considered here, we have adopted a coherent spin conserved tunneling approach $[48,24,47,107,106,146,147]$ where the total current is obtained simply by adding the spin-up and the spin-down currents. The results for the total spin-polarized current as a function of $\varepsilon_{g}$ for the PC and the APC are presented in Figs. 5.1a and $1 \mathrm{~b}$, respectively. The lead is assumed to have a single magnetic domain as shown in the inset of Figs. 5.1a and 1b. It is noteworthy to mention that in both cases the spin-down states are found to contribute significantly to the total current ( see Supporting 
Information). In the absence of $\varepsilon_{g}$, the current for the $\mathrm{PC}\left(I_{P C}\right)$ is found to be higher than the current for the APC $\left(I_{A P C}\right)$. A steady increase in current is noted for the bias up to $\sim 0.5 \mathrm{~V}$ beyond which a non-linear feature in the current is observed for both PC and APC. Remarkably, within the linear current regime, with the increase of $\varepsilon_{g}$, the $I_{P C}$ is found to decrease in contrast to the increase in $I_{A P C}$. A closer inspection shows a much stronger response to $\varepsilon_{g}$ in the $\mathrm{APC}$ compared to that in the PC. For example, at a small bias of 0.2 $\mathrm{V}$, there is a $16 \%$ decrease in the $I_{P C}$ compared to an increase of $221 \%$ in the $I_{A P C}$ when $\varepsilon_{g}$ increases from 0 to $2.04 \mathrm{~V} / \AA$. For a higher $\varepsilon_{g}$, the total currents for both PC and APC rise initially to reach peak values with the increase of $V_{s d}$ and then drop to valley points with the subsequent increase in $V_{s d}$ before increasing again, revealing clear NDR features. For $\varepsilon_{g}=2.04 \mathrm{~V} / \AA$, the peak to valley current ratio $\left(I_{p} / I_{v}\right)$ in the APC is found to be 1.7 ; for PC the $I_{p} / I_{v}$ is 1.4 .

Tunnel magnetoresistance. To quantify this surprisingly contrasting response between the PC and the APC to $\varepsilon_{g}$, we have calculated the TMR as $\left(I_{P C}-I_{A P C}\right) / I_{A P C}$. Fig. 5.3 summarizes the bias dependent TMR data for $\varepsilon_{g}=0.00 \mathrm{~V} / \AA ̊$ and $2.04 \mathrm{~V} / \AA$. In the absence of the transverse electric field, the signs of TMR values are found to be positive for all the bias points considered here. In contrast, for $\varepsilon_{g}=2.04 \mathrm{~V} / \AA$, the signs of TMR values are found to be negative. To elucidate this unique transverse electric field dependent TMR result, we have calculated the TMR as a function of $\varepsilon_{g}$ at a small bias of $0.2 \mathrm{~V}$ (Fig. 5.1c). A significant variation in TMR from $+23 \%$ to $-67 \%$ with the switching of sign at a critical electric field $(\sim 0.8 \mathrm{~V} / \AA)$ is noted. We have also performed spin-polarized current 

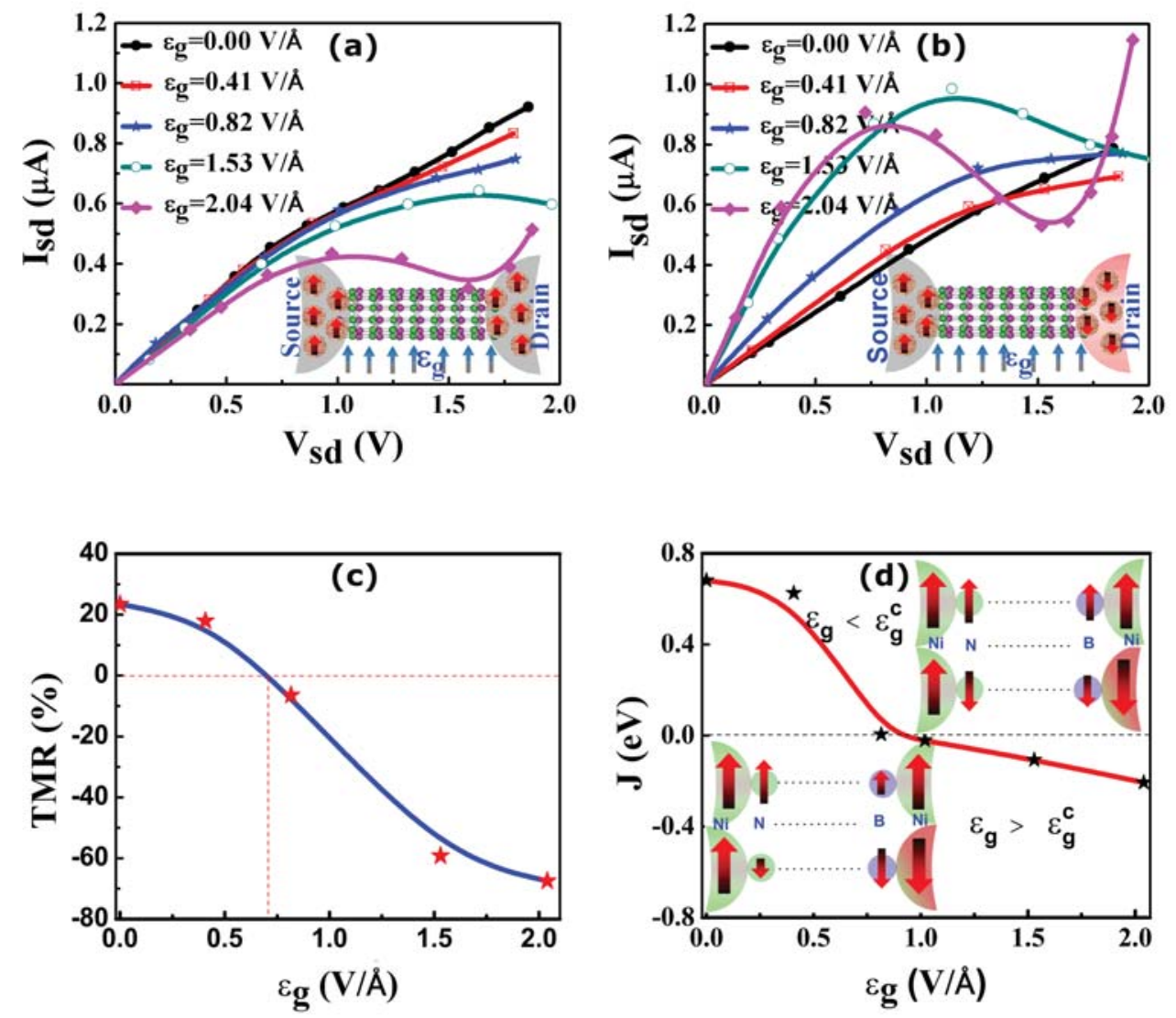

Figure 5.1: $I_{s d}-V_{s d}$ curves in a BNTQD tunnel junction for (a) PC and (b) APC as a function of $\varepsilon_{g}$. Insets show the schematic junction structures. (c) TMR vs. $\varepsilon_{g}$ at $V_{s d}$ of $0.2 \mathrm{~V}$. (d) Exchange coupling $(\mathbf{J})$ as function of $\varepsilon_{g}$. Inset shows the $\varepsilon_{g}$ dependent spin-profiles at the interfaces. The height and width of the arrow determine the magnitude of magnetic moment. Up and down arrows denote positive and negative magnetic moments respectively. Reprinted figure with permission from K. B. Dhungana and R. Pati, Phys. Chem. Chem. Phys. 16, 7996-8002 (2014). (c) The Owner Societies 2014.

calculation using a $(7,0)$ BNTQD channel of same length with the same interface distance to check whether the switching feature in TMR observed here persists for other diameters. Indeed, we have found a similar switching feature in TMR as observed for $(6,0)$ BNTQD, which confirms the general nature of our observations irrespective of the diameter of the 


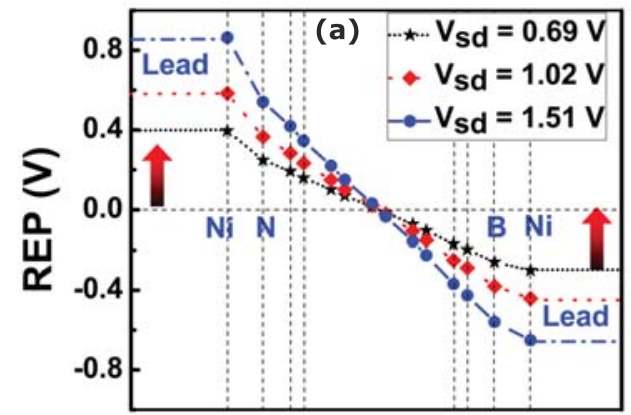

Position

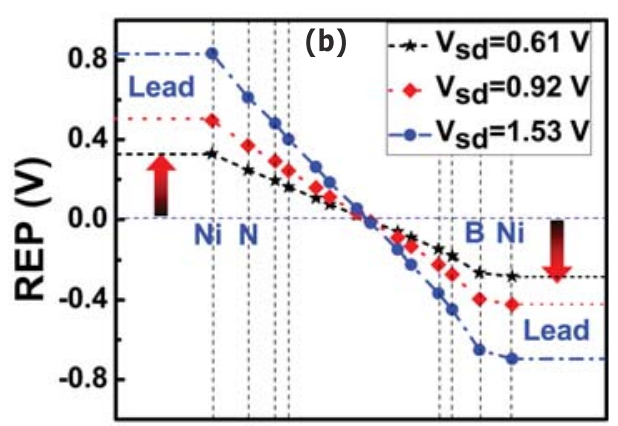

Postition

Figure 5.2: (a) for $\mathrm{PC}$ and (b) for APC. The vertical dotted lines represent the planar atomic position of the device along the direction of current; the horizontal dotted line refers to the equilibrium situation; REP refers to the potential drop with respect to the equilibrium. Reprinted figure with permission from Kamal B. Dhungana and Ranjit Pati, Phys. Chem. Chem. Phys. 16, 7996-8002 (2014). (c) the Owner Societies 2014.

tube. It should be noted that a significant diameter dependent band-gap modulation with $\varepsilon_{g}$ has been reported in pristine BNT $[148,149]$. The band-gap modulation has been shown to increase with the tube diameter $[148,149]$ and is found to be independent of chirality. This clearly suggests that a smaller critical field $\left(\varepsilon_{g}^{c}\right)$ than that found in our calculation would be suffice to switch the TMR in a BNTQD junction with larger diameter. In addition, the same order of transverse electric field as predicted here has been applied experimentally on BNT of diameter $16.3 \pm 6 \AA$ to observe giant Stark effect [144], which implies that our predicted critical field for switching TMR would be accessible to the experiment $[15,150]$. It is also worthwhile to note that a similar gate field dependent switching in the sign of TMR has been observed at low temperature in a CNTQD-magnetic tunnel junction device [130]. 

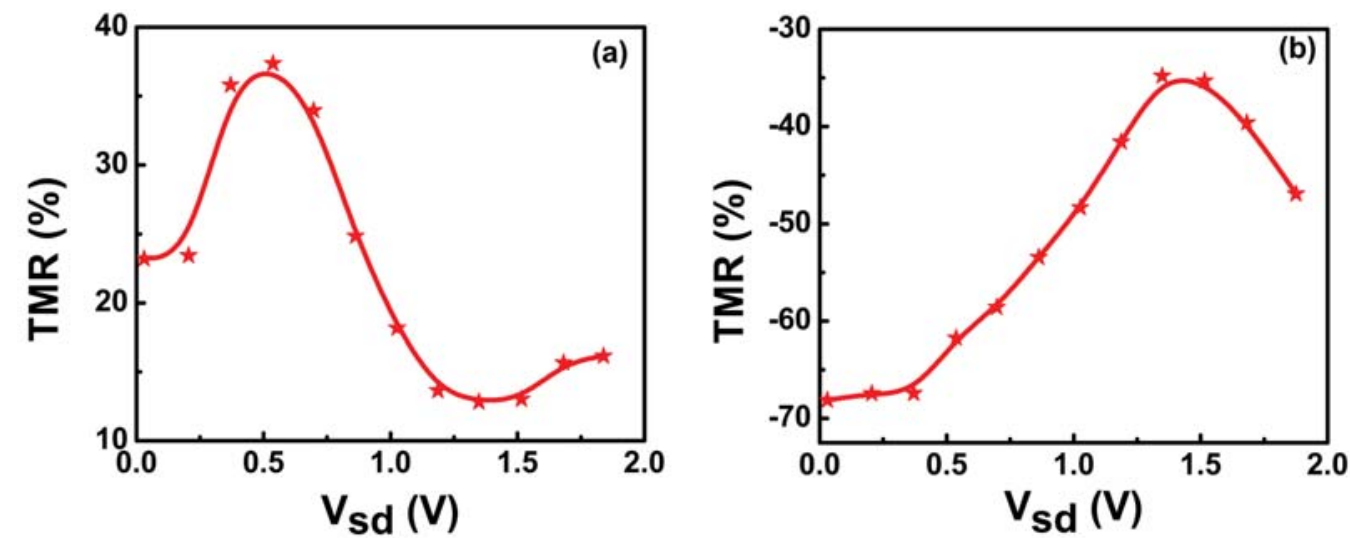

Figure 5.3: TMR as a function of applied bias $\left(V_{s d}\right)$ for (a) $\varepsilon_{g}=0.00 \mathrm{~V} / \AA$, and (b) $\varepsilon_{g}=$ 2.04 V/Å. Reprinted figure with permission from K. B. Dhungana and R. Pati, Phys. Chem. Chem. Phys. 16, 7996-8002 (2014). (c) The Owner Societies 2014.

Magnetic exchange coupling. To understand the origin of switching in the sign of TMR, the magnetic exchange coupling, $\mathbf{J}=E_{P C}-E_{A P C}$, is calculated as a function of $\varepsilon_{g}$ (Fig. 5.1d). $E_{P C}$ and $E_{A P C}$ are total energies for the PC and the APC respectively in the extended system. At zero $\varepsilon_{g}, \mathbf{J}$ is found to be positive with the APC being the more stable configuration. When we increase $\varepsilon_{g}$ from 0 to $2.04 \mathrm{~V} / \AA$, the value of $\mathbf{J}$ is found to decrease toward a negative value with the switching of sign at $\varepsilon_{g}$ of $\sim 0.8 \mathrm{~V} / \AA$. A strong correlation is found between the variation of $\mathbf{J}$ and TMR; the switching of $\mathbf{J}$ is found at a slightly higher $\varepsilon_{g}$ than TMR. This can be understood from the fact that $\mathbf{J}$ calculation does not consider the imaginary part of the Hamiltonian as incorporated in TMR calculation for the open device. To gain deeper insights into the cause of sign reversal in $\mathbf{J}$, we examine the spin profile of the device as a function of $\varepsilon_{g}$. Due to strong exchange interactions between the electrons at the interface, the non-magnetic BNTQD becomes spin polarized and the atoms that are 
in close proximity to $\mathrm{Ni}$ gain substantial magnetic property $[137,138]$; the interface now acts as a spin-interface $[139,47]$. Since we have B atoms at the one interface and $\mathrm{N}$ atoms at the other, there is an asymmetric spin profile at the interfacial atoms. For example, in the case of APC, the average magnetic moment per atom $(\bar{\mu})$ in the nitrogen layer at the close vicinity of $\mathrm{Ni}$ lead ( $\sim 1.3 \mu_{B}$ per atom) changes from $-0.12 \mu_{B}$ to $-0.07 \mu_{B}$ when we increase $\varepsilon_{g}$ from 0 to $2.04 \mathrm{~V} / \AA$; in the case of boron-nickel interface layer, $\bar{\mu}$ for boron changes from $-0.01 \mu_{B}$ to $-0.03 \mu_{B}$. For PC, $\bar{\mu}$ in the boron layer at the interface decreases from $0.16 \mu_{B}$ to $0.13 \mu_{B}$ by changing $\varepsilon_{g}$ from 0 to $2.04 \mathrm{~V} / \AA$; only a small change from $0.47 \mu_{B}$ to $0.48 \mu_{B}$ is noted for the $\bar{\mu}$ in the nitrogen layer at the interface. This spin profile at the interface is shown schematically in the inset of Fig. 5.1d. For $\varepsilon_{g}<\varepsilon_{g}^{c}$, the strong negative exchange interaction between $\mathrm{Ni}$ and $\mathrm{N}$ at the interface for the APC (favored by the Hund's rule) explains the stability of the anti-parallel configuration over the PC. For $\varepsilon_{g}>\varepsilon_{g}^{c}$, there is a substantial decrease in the magnetic moment of the $\mathrm{N}$ at the interface for the APC resulting in a lower negative exchange interaction at the $\mathrm{Ni} / \mathrm{N}$ spin-interface; at the same time, the magnetic moment of the B at the other interface increases leading to a stronger positive exchange interaction at the $\mathrm{Ni} / \mathrm{B}$ spin-interface. Conversely, for $\varepsilon_{g}>$ $\varepsilon_{g}^{c}$, a substantial decrease in the magnetic moment at the B for the PC leads to a weaker positive exchange interaction at $\mathrm{Ni} / \mathrm{B}$ spin-interface. This makes the $\mathrm{PC}$ more stable than the APC for $\varepsilon_{g}>\varepsilon_{g}^{c}$. Thus, unambiguously, we confirm that the electric field manipulation of spin-interface is the main cause for switching of $\mathbf{J}$ and TMR. Now the question arises: What is the mechanism that causes the change in spin profile at the interface between PC 
and APC ? To answer this, we calculate electric dipole moment $\alpha^{j}$ and polarizability $\beta^{j k}$ for PC and APC (shown in the Table I). Since the y-components of dipole moment and polarizability for $\mathrm{PC}$ and $\mathrm{APC}$ are distinct, each spin configuration responds uniquely to the transverse electric field due to Stark effect [136]. This, in fact, results in an energy cross over and switching of $\mathbf{J}$. Next, we turn our focus to another important factor, the spin injection co-efficient, $\eta$, which dictates the spin injection efficiency from $\mathrm{Ni}$ electrode to BNTQD. It should be noted that spin injection into a semiconductor from a ferromagnetic contact can be measured using spin-resolved two-photon photoemission technique [151]. We have calculated the bias dependent $\eta$ as [47]: $\eta=\left(I_{u p}-I_{d o w n}\right) /\left(I_{u p}+I_{d o w n}\right) ; I_{u p}$ and $I_{\text {down }}$ refer to the spin-up and spin-down components of the current, respectively. For PC, the maximum and minimum spin injection factors $\left(\eta_{\max }\right.$ and $\left.\eta_{\min }\right)$ are found to be -0.89 and -0.81, respectively (Fig. 5.4a). In APC, $\eta_{\max }$ and $\eta_{\min }$ are found to be -0.74 and -0.61 , respectively ( Fig. 5.4b). These high values of $\eta$ suggest that the Ni/BNTQD spin-interface acts as an natural spin-selective tunnel barrier for spin injection.

Spin dependent transmission. To further our understanding of the observed $\varepsilon_{g}$ dependent spin current behavior in PC and APC, we have analyzed the $T^{\sigma}\left(E, V_{s d}, \varepsilon_{g}\right)$. For brevity, the results for $T^{\sigma}$ at two representative $\varepsilon_{g} s$ ( 0 and $2.04 \mathrm{~V} / \AA$ ) are summarized in Figs. 5.5a and 5.5b. In both PC and APC, we find a substantially higher contribution to the transmission from the spin-down states, which explains the higher observed spin-down current. A significant broadening occurs in the spin-down case for both PC and APC, which can be inferred from the spilling of Ni spin-down density of states (SD-DOS) into 

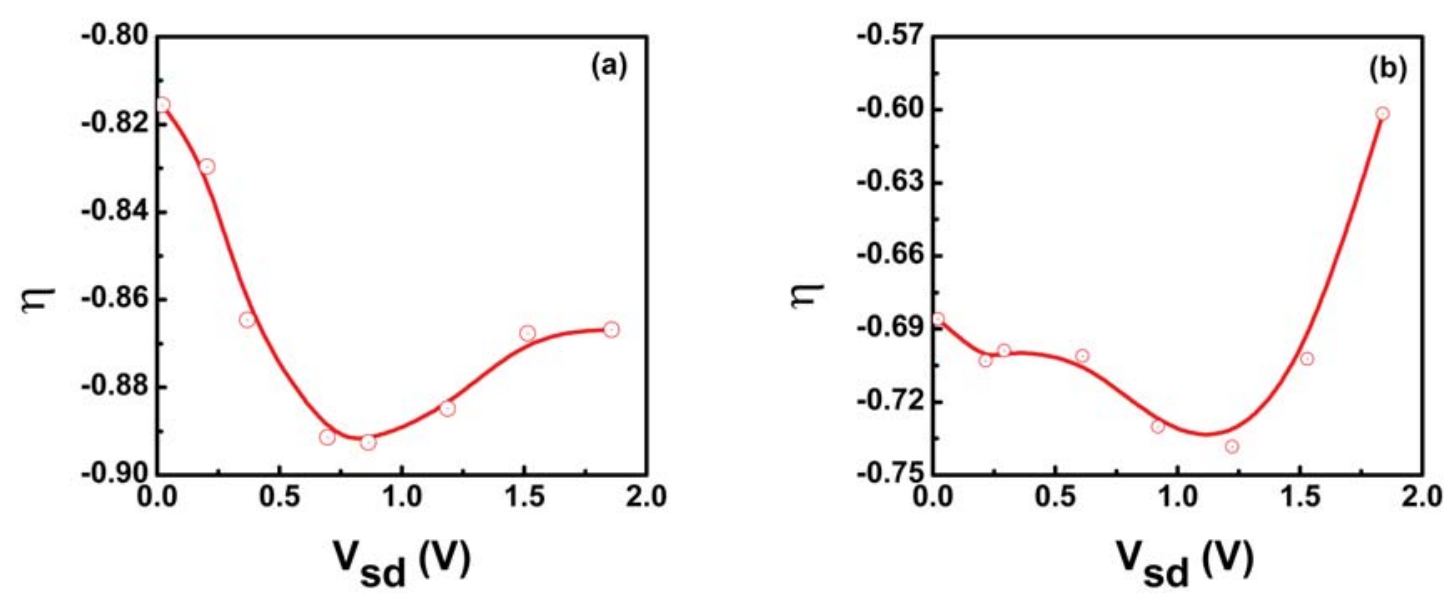

Figure 5.4: Bias dependent spin injection factor $(\eta)$ in the BNTQD-Ni tunnel junction for $\varepsilon_{g}=0 \mathrm{~V} / \AA$ : (a) parallel spin configuration (PC), and (b) anti-parallel spin configuration (APC). Since $I_{d o w n}>I_{u p}$, the $\eta$ is found to be negative. Reprinted figure with permission from Kamal B. Dhungana and Ranjit Pati, Phys. Chem. Chem. Phys. 16, 7996-8002 (2014). (c) The Owner Societies 2014.

the BNTQD due to the strong coupling at the interface and a much higher SD-DOS of the Ni-lead at the Fermi energy. The asymmetry in $T^{\sigma}$ between spin-up and spin-down states for the APC is expected due to intrinsic structural asymmetry at the interface and the bias induced electronic asymmetry. Transmission data show a much weaker response to $\varepsilon_{g}$ in the case of PC as compared to the APC, which is also reflected in their respective total spin-polarized currents. A closer inspection of Figs. 5.5a and 5.5b reveals that the height of the transmission feature near the Fermi energy decreases for the PC with the increase of $\varepsilon_{g}$ (Fig. 5.5a). In contrast, a substantial increase in the height of the transmission feature is observed for the APC near the Fermi energy with increasing $\varepsilon_{g}$ (Fig. 5.5b). This clearly explains the observed decrease in current for the PC compared to an increase in current for 
the APC at a smaller bias with increasing $\varepsilon_{g}$. In the absence of $\varepsilon_{g}$, the transmission height for the PC in the vicinity of the Fermi energy is higher than that in APC resulting a higher $I_{P C}$ than $I_{A P C}$ (Figs. 5.1a and 5.1b).
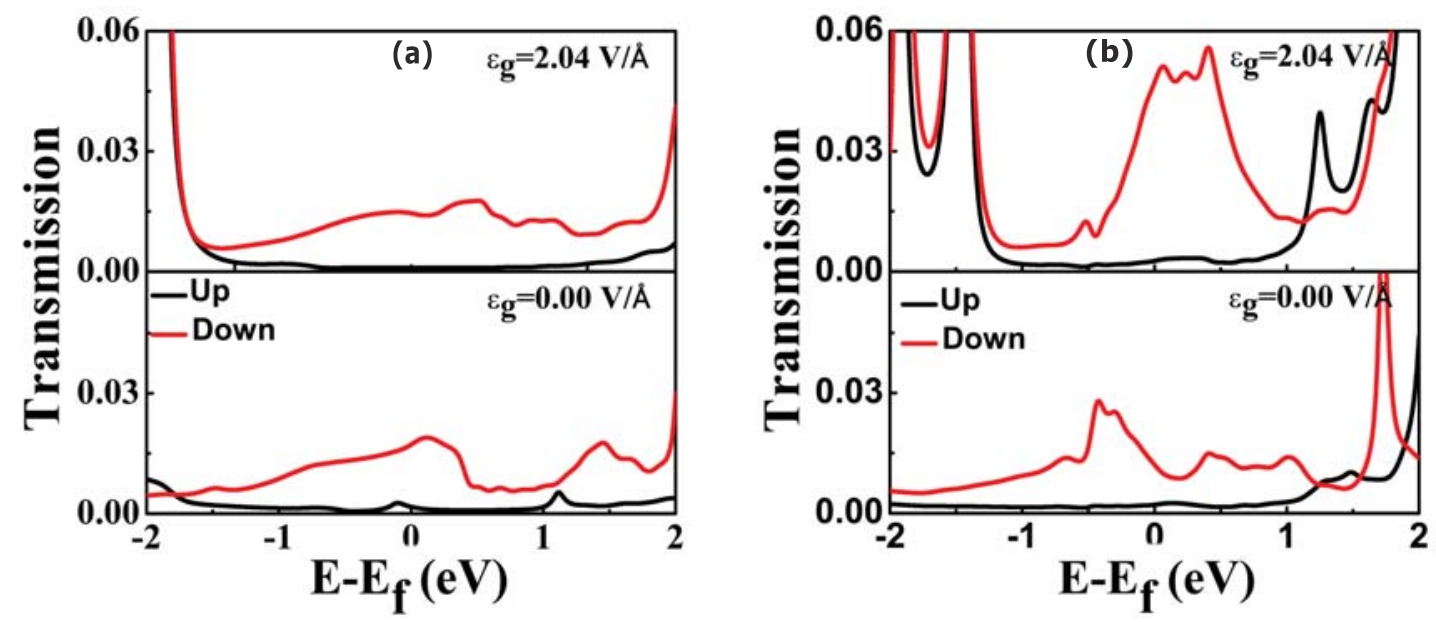

Figure 5.5: $\varepsilon_{g}$ dependent spin-polarized transmission for (a) parallel spin configuration and (b) anti-parallel spin configurations at $V_{s d}$ of $\sim 0.2 \mathrm{~V}$. Reprinted figure with permission from K. B. Dhungana and R. Pati, Phys. Chem. Chem. Phys. 16, 7996-8002 (2014). (c) The Owner Societies 2014.

Nonlinear spin-polarized current. Now the question arises: What is the cause for the strong non-linear NDR behavior in the spin-polarized current at a higher $\varepsilon_{g}$ ? To answer this subtle question, we have looked at the $T^{\sigma}$ at $\varepsilon_{g}=2.04 \mathrm{~V} / \AA$ for both the PC and the APC. Since the APC shows a much stronger non-liner response at a higher $\varepsilon_{g}$, we have summarized the results for only the APC ( Fig. 5.6a); only four bias points are considered. The transmission height decreases within the chemical potential window $(\mathrm{CPW})$ for the spin-down states as $V_{s d}$ increases from $0.35 \mathrm{~V}$ to $0.72 \mathrm{~V}$. However, since the width of the 
$\mathrm{CPW}$ is much higher for $V_{s d}=0.72 \mathrm{~V}$ and the current is dictated by the area under the transmission curve within the $\mathrm{CPW}$, we observe a higher current at $V_{s d}=0.72 \mathrm{~V}$. When we increase the bias further to $V_{s d}=1.51 \mathrm{~V}$, in spite of the increase in CPW width, we see a substantial drop in the height of the transmission within CPW resulting in a significant drop in current leading to a NDR feature. A similar drop in transmission with increase in bias leading to NDR feature has been observed in molecular junction [26]. It should be pointed out that $V_{s d}=0.72 \mathrm{~V}$ and $1.51 \mathrm{~V}$ correspond to the peak-current and valley-current position respectively for the APC. When we increase $V_{s d}$ to $1.92 \mathrm{~V}$, the spin-up states start contributing significantly within the CPW leading to an increase in total current. The next question is: Why do we see a significant drop in transmission with the increase in $V_{s d}$ ? We examine the bias dependent spin orbital for the APC and its response to $\varepsilon_{g}$ to answer this inquiry. One of the frontier spin-down orbitals (i.e the highest occupied orbital of the active scattering region, $\mathrm{HOMO}$ ) that contributes to the transmission within the $\mathrm{CPW}$ is presented in the inset of Fig. 5.6a. A dramatic transformation in the shape of the spin orbital is noticeable as $V_{s d}$ changes from $0.35 \mathrm{~V}$ to $1.51 \mathrm{~V}$. For $V_{s d}=0.35 \mathrm{~V}$, the electron (spin-down) cloud is distributed at both the interfaces despite some asymmetry in distribution between the two interfaces. With the increase of $V_{s d}$, the asymmetry in electron density distribution between two interfaces increases; at $V_{s d}=1.51 \mathrm{~V}$ (valley point), due to the strong field induced orbital mixing, the electron cloud is distributed only at the $\mathrm{Ni} / \mathrm{B}$ interface resulting in a smaller $T^{\sigma}$, and hence $I^{\sigma}$. 
Table 5.1

Components of dipole moment $(\alpha)$ and polarizability $(\beta)$ for parallel spin configuration (PC) and anti-parallel spin configuration (APC). Reprinted table with permission from K.

B. Dhungana and R. Pati, Phys. Chem. Chem. Phys. 16, 7996-8002 (2014). (C) The Owner Societies 2014.

\begin{tabular}{|l|l|l|l|l|l|l|l|l|l|}
\hline & \multicolumn{6}{|c|}{ Dipole moment(a.u) } & \multicolumn{5}{|c|}{ Polarizability(a.u) } \\
\hline comp. & $\alpha^{x}$ & $\alpha^{y}$ & $\alpha^{z}$ & $\beta^{x x}$ & $\beta^{y x}$ & $\beta^{y y}$ & $\beta^{z x}$ & $\beta^{z y}$ & $\beta^{z z}$ \\
\hline PC & 0.08 & 0.14 & 9.59 & 692.78 & -006.03 & 692.53 & 042.62 & -000.72 & 5215.20 \\
\hline APC & 0.95 & 0.79 & 8.58 & 677.26 & -006.17 & 675.82 & -022.72 & 122.57 & 5898.05 \\
\hline
\end{tabular}

Stark effect. To understand the non-linear response at higher $\varepsilon_{g}$ in greater detail, we then examine the Stark shift for both PC and APC for the extended system (Fig. 5.6b). The Stark shift is calculated as: $\delta E^{n}=\varepsilon_{g}^{n}\left(V_{s d}\right)-\varepsilon_{g}\left(V_{s d}=0\right)$, where $n$ corresponds to different participating spin orbitals and $\varepsilon_{g}$ is the energy of the spin orbital in the presence of $\varepsilon_{g}$ and $V_{s d}$. $\mathrm{H} 0$ - the energy of the spin-down HOMO at equilibrium — is considered as the reference energy, $\varepsilon_{g}\left(V_{s d}=0\right)$. Since spin-down states dictate the behavior of the current, we have presented the results only for the spin-down states at $\varepsilon_{g}=0$ and $2.04 \mathrm{~V} / \AA$. A strong non-linear Stark shift $\left(\sum_{j} \alpha^{j} \varepsilon_{g}^{j}+\frac{1}{2} \sum_{j, k} \beta^{j k} \varepsilon_{g}^{j} \varepsilon_{g}^{k}+\ldots \ldots \ldots\right)$ for the frontier orbitals at a higher bias is noticeable at $\varepsilon_{g}=2.04 \mathrm{~V} / \AA$. Each spin orbital responds differently to $\varepsilon_{g}$ as each orbital has a unique electron density distribution with distinctive dipole moment and polarizability. APC is found to exhibit a much stronger response to the transverse electric field (energy level spacing between $\mathrm{H} 0$ and $\mathrm{H} 1$ decreases significantly) than the PC. This can also be inferred from the calculated dipole moment and polarizability (Table I). In the case of the APC, $\alpha^{y}$ and $\beta^{z y}$ (components along the transverse field direction) are much stronger than that in PC. 

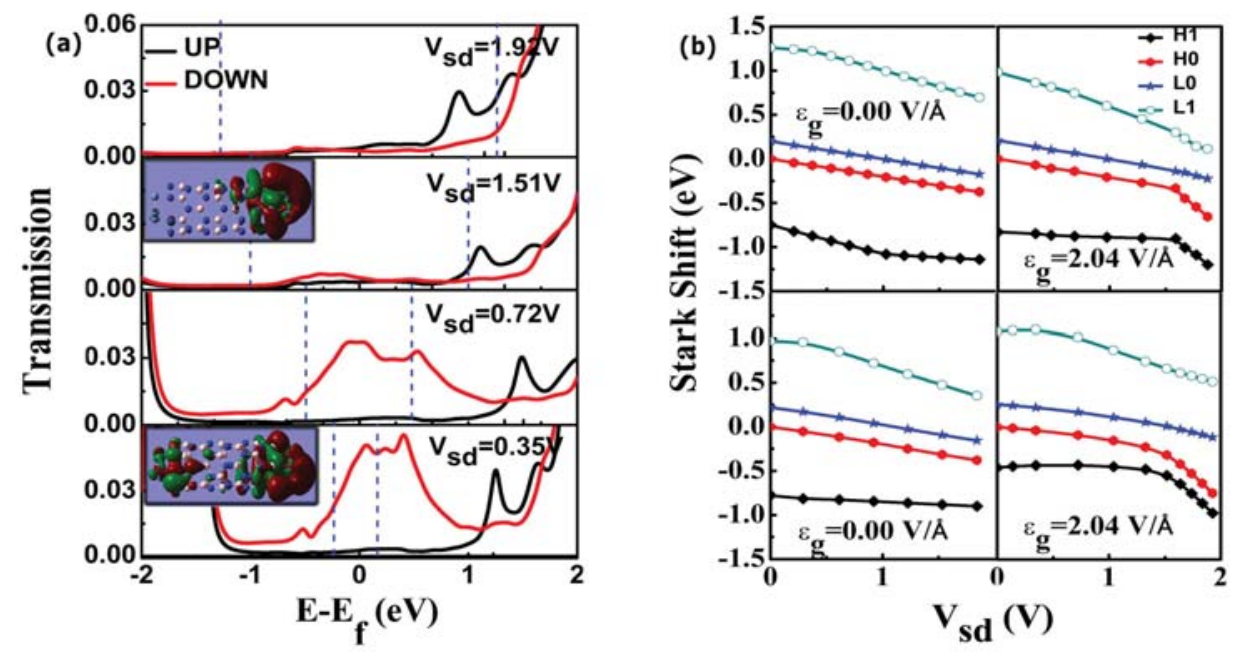

Figure 5.6: (a) Bias dependent spin-polarized transmission for the APC of the extended system at $\varepsilon_{g}=2.04 \mathrm{~V} / \AA$. Dotted lines represent the chemical potential window $(\mathrm{CPW})$. The inset shows the bias dependent spin-down HOMO for the APC at $\varepsilon_{g}=2.04 \mathrm{~V} / \AA$. (b) Bias dependent Stark shift corresponding to the frontier spin-down orbitals as a function of $\varepsilon_{g}$. Upper two panels are for PC and lower two panels are for APC. H0, H1 refer to the HOMO and HOMO-1, and L0, L1 refer to the LUMO and LUMO+1 spin-down orbitals in the extended system. Reprinted figure with permission from K. B. Dhungana and R. Pati, Phys. Chem. Chem. Phys. 16, 7996-8002 (2014). (c) The Owner Societies 2014.

\subsection{Conclusions}

We have demonstrated electrical manipulation of quantum spin state of the electron in a BNTQD-magnetic tunnel junction device to show switching in the sign of exchange coupling and tunnel magnetoresistance. Most importantly, the switching feature in tunnel magnetoresistance observed here is found to be independent of the diameter of the BNTQD channel, confirming the general nature of our prediction. Electric field induced Stark effect 
causing a change in magnetic exchange interaction at the interface is found to be the main mechanism behind the switching in sign. In addition, we have observed a very high spin injection efficiency from nickel electrode to BNTQD. We expect the magnitude of the critical electric field for switching the sign of tunnel magnetoresistance to decrease with the increase in diameter of the BNTQD as revealed from the band-gap modulation study in pristine BNT. Since the predicted external electric field for switching the sign of tunnel magneto-resistance is within the range accessible to the experiment, we expect our findings would open up new initiatives for the application of the BNTQD tunnel device in next generation spin based nano-scale electronics. 


\section{Chapter 6}

\section{Fluorine Functionalized Boron Nitride}

\section{Nanotube for Spintronics*}

\subsection{Introduction}

A boron nitride nanotube (BNNT) $[152,153,143]$ is an inorganic analogous of carbon nanotube (CNT) [154]. Unlike in CNT, the electronic structure is independent to chirality in BNNT $[142,144,155]$. However, the observed large band gap and high electrical resistivity hinder its application in nano-electronics [156]. Over the past three decades, several attempts have been made to tune the band gap of BNNT [156]. For example, the transverse

*Portion of this chapter is copied from the Journal of American Chemical Society vol. 136, page 11494-11498, year 2014, and Sensors vol. 14, page 17655-176585, year 2014 by K. B. Dhungana and R. Pati. Copyright - Appendix C. DOI: 10.1021/ja505757f \& DOI:10.3390/s140917655 
electric field is utilized for modulating the band gap; the electric field reduces the band gap significantly [144]. This effect is noticed to be diameter dependent [148, 149]. Similarly, researchers have used functionalization and doping of BNNT with different foreign species for tuning the band gap of BNNT; foreign species, such as atoms, molecules, and metallic clusters, have been used [156]. The functionalization of BNNT with fluorine atoms is found to enhance the conductivity of BNNT and has received the attention in recent years $[157,158,159,160,161]$.

In a pioneering attempt, Tang et al. reported the synthesis of fluorinated BNNTs using a novel technique, where they introduced fluorine atoms during the tube growth [157]. They found the resistivity of the F-BNNT to be about three order of magnitude smaller than that of the pristine BNNT; the fluorinated BNNT has also been shown to exhibit p-type semiconducting behavior [158]. Soon after the experimental realization, based on a first principles approach, Li and co-workers first showed that the chemisorption of fluorine can induce spontaneous magnetization [159]. In another instance, Zhang et al. have shown for the first time that fluorine atoms adsorbed on BNNT can induce long-ranged ferromagnetic spin ordering along the tube [160]. Thus, it is evident that the fluorinated BNNT is a novel metal free magnetic entity with high conductivity, which makes it a suitable candidate for applications in spin based electronics (spintronics) [161]. It should be noted that the quest for novel metal free magnetic materials, which exhibit magnetism at a higher temperature, has been intensively pursued since the discovery of magnetic polymerized $\mathrm{C}_{60}$ [162]. Despite the desirable properties of fluorinated BNNTs for applications in 
spintronics, the spin dependent transport properties in the F-BNNTs metal junctions are not yet explored. Understanding the spin dependent current in such a junction not only depends on the intrinsic electronic and magnetic properties of F-BNNT but also on the electronic structure of the fluorinated BNNT-lead interface.

In this chapter, using a first principles approach, we unambiguously confirm the ferromagnetic ordering of spins in the fluorinated BNNT and show that the chemisorption of fluorine on a BNNT quantum dot with a coverage of $4.1 \%$ changes the conductance from $6.2 \mathrm{nS}$ to $3.9 \mu \mathrm{S}$, which is in very good agreement with the experimental observation. Most importantly, our first principles quantum transport study predicts that the F-BNNT quantum dot can act as a perfect spin filter with efficiency of $99.8 \%$. Further confinement of electrons in the F-BNNT quantum dot by passivating the two open ends of the channel with hydrogen atoms (weakly coupled junction) leads to additional enhancement of the spin filter efficiency. A minimal change in spin filter efficiency is found with the change in channel length; the change in F-coverage from $4.1 \%$ to $8.2 \%$ is found to alter the spin filter efficiency by a small amount from $99.8 \%$ to $99.1 \%$. These results thus suggest that the observed high spin filter efficiency is a general feature of the F-BNNT.

The rest of the chapter is organized as follows. In section 6.2, we will briefly describe our computational method. Results and discussions are presented in section 6.3 , followed by a brief summary in section 6.4 . 


\subsection{Computational Method}

Since BNNTs prefer to adopt a zigzag structure during experimental growth $[163,164]$, we have considered a representative zigzag BNNT of chirality $(6,0)$ for our study. A periodic density functional theory that employs plane wave basis functions and uses generalized gradient approximation (PW91) for the exchange-correlation is used for the electronic structure calculation of the pristine BNNT. The projected augmented wave (PAW) approach is used to describe the valence-core interaction. We use $1 \times 1 \times 11 k$ point grid within the Monkhorst-Pack (MP) scheme to sample the Brillouin zone. Calculations are performed using the Vienna ab initio simulation (VASP) code [165]. The structure is considered optimized when the force on each atom is $\leq 0.01 \mathrm{eV} / \AA$; the convergence criterion for the energy is $10^{-6} \mathrm{eV}$. For the fluorinated BNNT, we have used the spin polarized DFT and have considered a $4.1 \%$ coverage of fluorine i.e. one fluorine atom in the super cell comprising of twenty-five atoms. Our choice of a small F coverage of $4.1 \%$ is prompted by a similar low coverage used in the experiment $[157,158]$.

To study the electron transport property of the fluorinated BNNT, we have constructed a prototypical device by sandwiching a fragment of the F-BNNT $(\sim 1.5 \mathrm{~nm}$ length $)$ between two gold electrodes (Fig. 6.1). It should be noted that the experimental measurement of current in the F-BNNT is carried out with gold electrodes [157]. Since electrons are

confined in the F-BNNT channel and there is a lattice mismatch between the gold electrode 


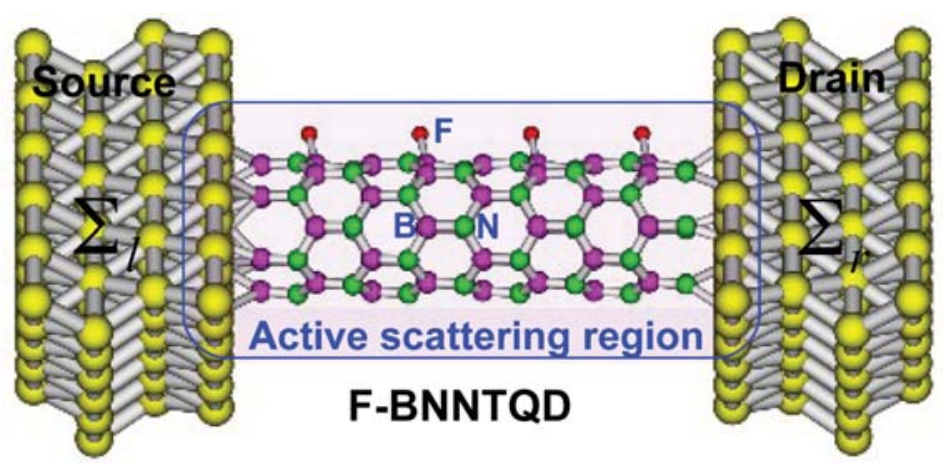

Figure 6.1: Schematic diagram of a spin-filter with F-BNNTQD as the channel. $\Sigma_{l}$ and $\Sigma_{r}$ are the self energy functions that allow the F-BNNTQD to exchange energy and electrons with the semi-infinite gold electrodes of the open device. Reprinted figure with permission from K. B. Dhungana and R. Pati, J. Am. Chem. Soc. 136, 11494-11498 (2014). (C) The American Chemical Society.

and F-BNNT, we term the channel hereafter as the F-BNNT quantum dot (QD). Since the F-BNNTQD is magnetic in nature, we have adopted a real space approach that allows us to construct the spin polarized retarded Green's function $\left(G^{\sigma}\right)[47,127,161]$ of the open device by dividing it into two parts: (i) the active scattering region consisting of the NTQD together with a finite number of Au atoms from the lead (Fig. 6.1) and (ii) the rest of the electrode on each side which is assumed to retain its bulk (3D) property. Subsequently, a spin conserved tunneling approach as developed in ref. 92 is used to calculate the spin dependent current in the F-BNNTQD. Spin up (majority) and spin down (minority) contributions are same in the non-magnetic, pristine BNNTQD-gold junction. A posteriori B3LYP functional, which eliminates partly the self-interaction error by including the exact exchange energy from the Hartree-Fock approach, is used to calculate the electronic 
structures. The LANL2DZ effective core-potential basis set that includes scalar relativistic effects is used to describe the gold atoms, whereas other relatively light atoms $(\mathrm{F}, \mathrm{B}, \mathrm{N})$ are represented by the all electron $6-311 \mathrm{G}^{*}$ basis set [126]. A finite perturbative approach is used to include the bias effect self-consistently.

\subsection{Results and Discussions}

\subsubsection{Electronic structure}

First, we focus on the electronic structure calculations of the pristine and fluorinated BNNTs. Our calculation yields a direct band gap of $2.77 \mathrm{eV}$ in the pristine BNNT, which is in line with the previous report [166]. In the case of the F-BNNT, we have considered the most stable exohedral binding of a fluorine atom on top of a boron atom, which has been reported to be the most favorable configuration $[159,160,167,158]$. The binding energy $E_{b}$, defined as $E_{b}=\left[E_{B N N T}+E_{F}-E_{F-B N N T}\right]$ for this most stable configuration, is found to be $3.02 \mathrm{eV} ; E_{B N N T}, E_{F}$, and $E_{F-B N N T}$ are the energies of the pristine BNNT, F atom, and fluorinated BNNT, respectively. Structure optimization of the F-BNNT shows a F atom bringing the B atom to which it is bonded out of the surface, resulting in an increased B-N bond lengths near the adsorption site.

This local deformation changes the $\mathrm{sp}^{2}$ hybridization of a $\mathrm{B}$ atom in the pristine BNNT 

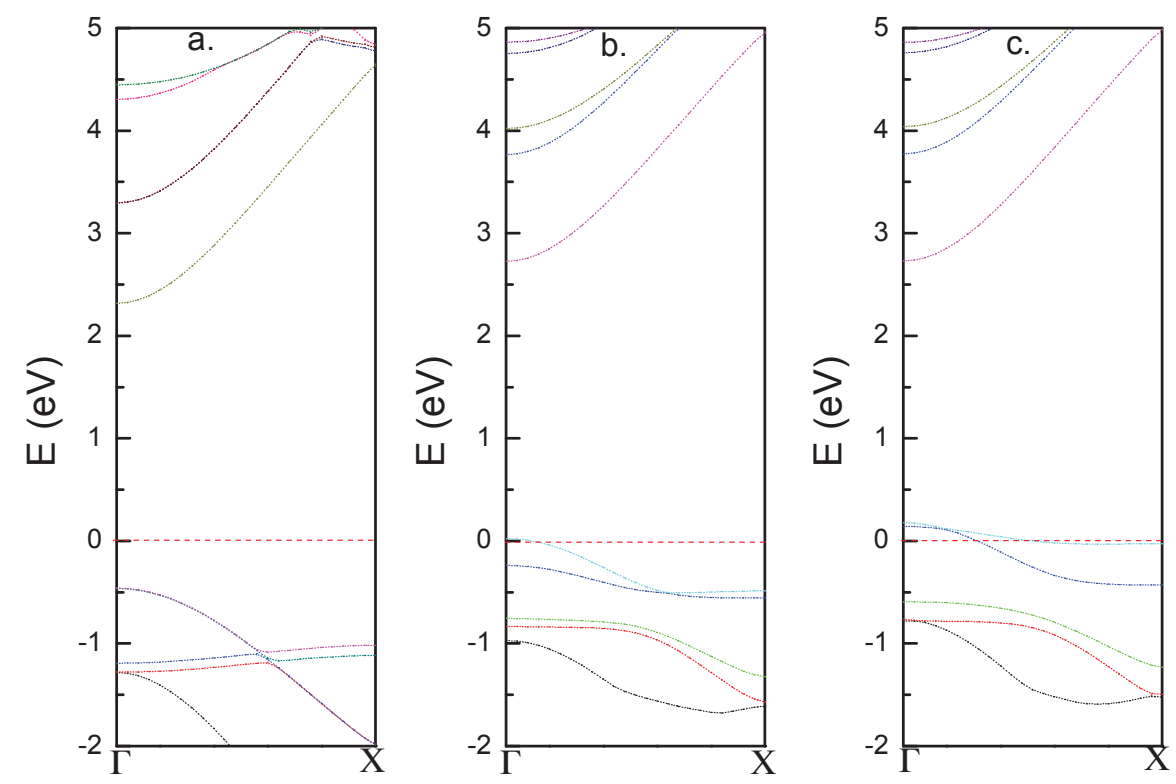

Figure 6.2: Electronic band structure of (a) pristine $(6,0)$ BNNT, (b) fluorinated (6, 0) BNNT (spin up states), and (c) fluorinated $(6,0)$ BNNT (spin down states); the Fermi-energy is set to zero. Reprinted figure with permission from K. B. Dhungana and R. Pati, Sensors 14, 17655-17686 (2014). (C) K. B. Dhungana and R. Pait.

to the $\mathrm{sp}^{3}$ hybridization in the F-BNNT. A similar fluorine induced hybridization change for the $\mathrm{C}$ atom has been observed in the F-CNT [168]. For a $4.1 \%$ fluorine coverage, the fluorinated BNNT is found to be magnetic with a total magnetic moment of $1.0 \mu_{B}$ for one F-atom in the unit cell; the unpaired electrons at the $\mathrm{N}$ atoms in the vicinity of the adsorbed $\mathrm{F}$ atom created by the loss of some electrons from it to the highly electronegative $\mathrm{F}$ atom contribute to the magnetism.

To analyze the presence of magnetism in F-BNNT, we have also calculated the band 
structures of pristine and fluorinated BNNT, which are shown in Fig. 6.2. No energy levels are crossing the Fermi-energy in pristine BNNT (Fig. 6.2a). However, partially occupied energy levels appear in the F-BNNT; Figs. $6.2 \mathrm{~b}$ and $6.2 \mathrm{c}$ shows the majority and minority bands in F-BNNT, respectively. The dispersion in induced energy levels in F-BNNT due to fluorine functionalization is small, which shows that the induced energy states are highly localized. The comparison of majority and minority band structures for F-BNNT at Fermi-energy shows that the density of state (DOS) for majority and minority spin states at Fermi-energy are different; as a result this system becomes magnetic [159, 160].

\subsubsection{Ferromagnetic spin ordering and thermodynamic stability}

We double the size of the supercell with the same coverage of $\mathrm{F}$ and perform spin polarized electronic structure calculations to check for the stability of the ferromagnetic ordering in the fluorinated BNNT. The ferromagnetic (FM) configuration is found to be more stable ( by $15 \mathrm{meV}$ ) than the antiferromagnetic configuration (AFM), which is in excellent agreement with the previously reported value [160].

To check for the thermodynamic stability of the FM state, we have calculated the energy barrier by finding the energy difference between the metastable paramagnetic state (expected transition point between the FM and AFM states) and the stable FM state. The

energy barrier is found to be $1.6 \mathrm{eV}$, which is much higher than the room temperature 
(26 meV). To examine if this FM behavior of F-BNNT persists for a higher F coverage, we increase the F-coverage to $16.4 \%$ by including four F-atoms in the unit cell; super cell used for our calculations consists of two unit cells with eight $\mathrm{F}$ atoms. The FM configuration is found to be much more stable (by $0.11 \mathrm{eV}$ ) than the AFM configuration as noted for the 4.1 $\%$ F-coverage. However, the barrier height between the FM and AFM states decreases to $0.61 \mathrm{eV}$. Though the barrier height for the $16.4 \%$ F-coverage is smaller than that observed for the $4.1 \%$ F-coverage, it is still much higher ( $\sim 23$ times) than the room temperature. This result thus ensures that the stable FM state in F-BNNT can not be inadvertently switched to the AFM state at a higher temperature - an important characteristic of a metal free magnetic entity. It is important to note that the magnetic property of the fluorinated BNNT is different from the fluorinated BN sheet. In the F-BN sheet, both the FM and AFM states are found to be nearly degenerate [169]. Curvature induced stress in the F-BNNT is responsible for the ferromagnetic ordering [169].

\subsubsection{Current-Voltage characteristics}

Next, we turn to current (I) - Voltage (V) calculations in the BNNT and F-BNNT quantum dots, which is the main focus of the present investigation. Fig. 6.3 summarizes the calculated I - V characteristics. Since the F-BNNT is magnetic, the total current for this system is obtained by adding the spin-up (majority) current $\left(I_{\uparrow}\right)$ and spin-down (minority) current $\left(I_{\downarrow}\right)$. The current in the F-BNNTQD is found to be significantly higher than that 


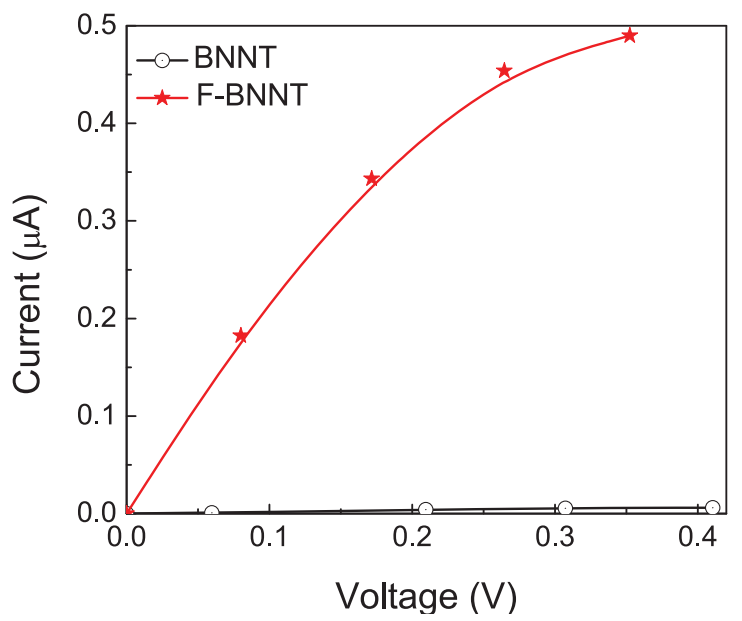

Figure 6.3: I-V characteristics of the pristine BNNTQD and F-BNNTQD coupled to the metallic gold electrodes; channel length is $\sim 1.5 \mathrm{~nm}$. Reprinted figure with permission from Kamal B. Dhungana and Ranjit Pati, J. Am. Chem. Soc. 136, 11494-11498 (2014). (C) The American Chemical Society.

of the pristine BNNTQD at the same bias. For example, at $\mathrm{V}=0.06 \mathrm{~V}$, the current for the F-BNNTQD is found to be $0.13 \mu \mathrm{A}$, which is 153 times higher than that of the pristine BNNTQD. This result is in good agreement with the experimental report [157], which shows a three order lower resistance in the fluorinated BNNT as compared to that in the pristine BNNT.

\subsubsection{Spin-injection efficiency and transmission}

To gain a deeper understanding of the observed higher current in F-BNNTQD, we examine up $(\uparrow)$ and down $(\downarrow)$ spin states contributions to the total current (Fig. 6.4). $I_{\downarrow}$ is found to be 


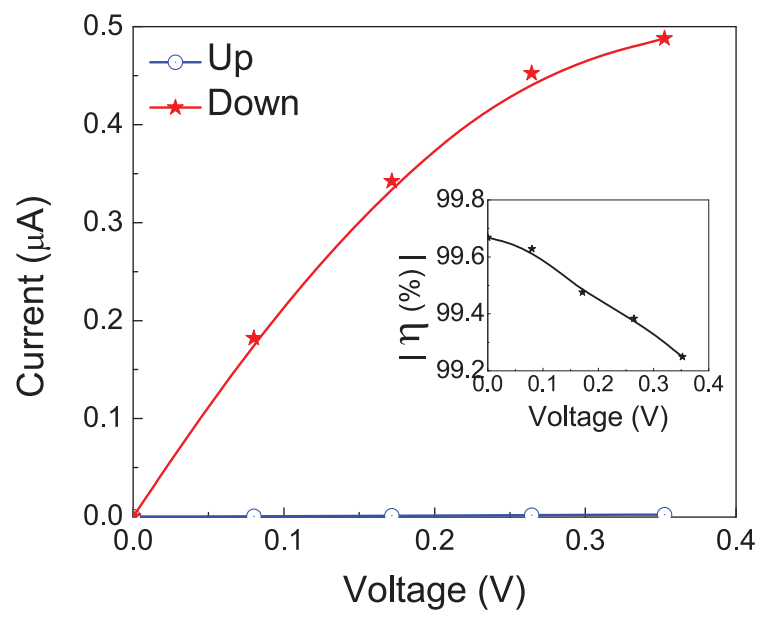

Figure 6.4: the variation of the magnitude of spin injection coefficient $(\eta)$ with bias; channel length is $\sim 1.5 \mathrm{~nm}$; Up and Down refer to majority and minority spin states, respectively. Reprinted figure with permission from K. B. Dhungana and R. Pati, J. Am. Chem. Soc. 136, 11494-11498 (2014). (c) The American Chemical Society.

appreciably larger than $I_{\uparrow}$. To quantify the difference between $I_{\uparrow}$ and $I_{\downarrow}$ in the F-BNNTQD, we have calculated the spin injection factor $(\eta)$ as $\left(I_{\uparrow}-I_{\downarrow}\right) /\left(I_{\uparrow}+I_{\downarrow}\right)$. The inset of Fig. 6.4 shows the variation of magnitude of $\eta$ as a function of applied bias. Within the bias range considered here, a small variation $(\sim 0.4 \%)$ of $\eta$ with bias is noted. The maximum magnitude of the $\eta$ value is found to be $99.76 \%$. It should be noted that for zero bias, the $\eta$ value is obtained as $\left(T_{\uparrow}\left(E_{f}\right)-T_{\downarrow}\left(E_{f}\right)\right) /\left(T_{\uparrow}\left(E_{f}\right)+T_{\downarrow}\left(E_{f}\right)\right) \times 100 \%$, where $T_{\uparrow}$ and $T_{\downarrow}$ are the transmission coefficients for spin-up and spin-down channels, respectively (Fig. 6.5); the zero bias $\eta$ value is referred to as the spin filter efficiency. To see how the length of the F-BNNT channel affects the spin filter efficiency, we have re-calculated the spin dependent transmission for a longer channel length of about $2 \mathrm{~nm}$ (Fig. 6.6). The same interface 
geometry as that of the shorter channel length is considered.

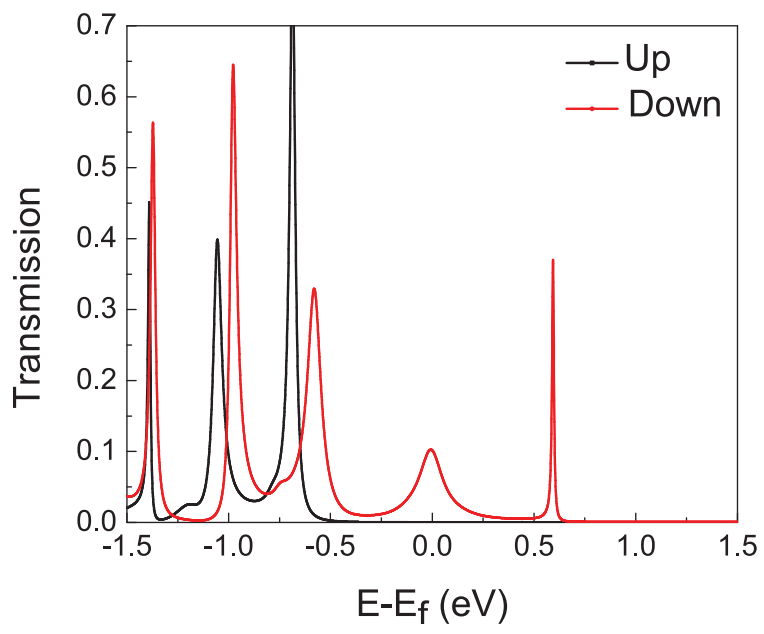

Figure 6.5: Spin dependent transmission in the F-BNNTQD-gold junction; the F-coverage is $4.1 \%$ and the channel length is $\sim 1.5 \mathrm{~nm}$; Up and Down refer to majority and minority spin states, respectively. Reprinted figure with permission from K. B. Dhungana and R. Pati, J. Am. Chem. Soc. 136, 11494-11498 (2014). (c) The American Chemical Society.

Though, the height of the transmission values for both the up and down spin states decrease at the Fermi energy for the longer channel length as expected in the tunneling regime, the spin filter efficiency is found to be $99.9 \%$, which is much closer to that observed for the shorter channel length. Thus, this result confirms that the observed high spin filter efficiency is a general feature of the F-BNNT channel (within the limit of spin coherence length). The observed high value of $\eta$ together with a very small variation with bias (see inset of Fig. 6.4) makes the F-BNNTQD a potential spin filter candidate. We have also calculated the spin dependent transmission for a $8.2 \%$ coverage of $\mathrm{F}$ on the BNNT to examine the dependence of $\mathrm{F}$ adsorbates on the spin filter efficiency. The results are 


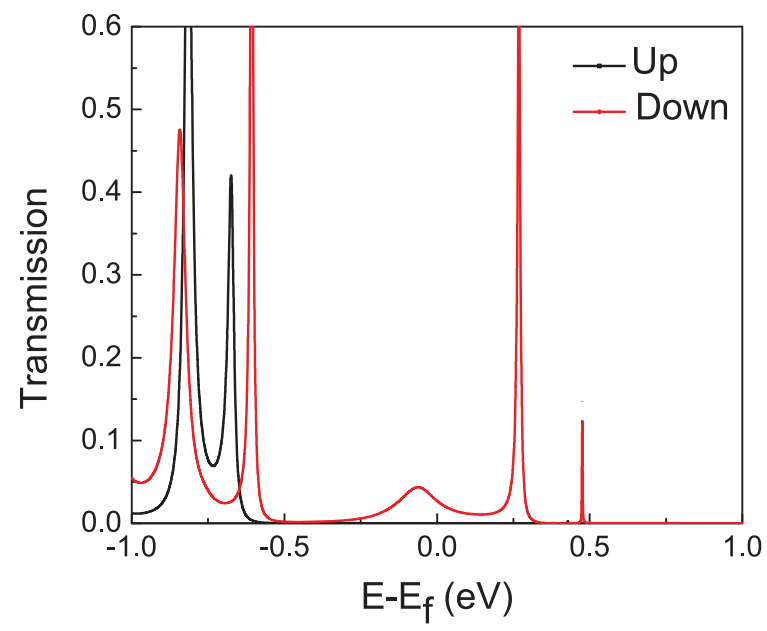

Figure 6.6: Spin dependent transmission in the F-BNNTQD-gold junction for the channel length of $\sim 2 \mathrm{~nm}$ and F-coverage of $4.1 \%$; Up and Down refer to majority and minority spin states, respectively. Reprinted figure with permission from K. B. Dhungana and R. Pati, J. Am. Chem. Soc. 136, 11494-11498 (2014). (c) The American Chemical Society.

shown in Fig. 6.7. Despite some noticeable changes in transmission features around the Fermi energy between the QDs with 4.1\% and 8.2\% F-coverage, the transmission from the minority spin states for the $8.2 \%$ F-coverage is found to be significantly higher than that of the majority spin states at the Fermi energy as noted for the $4.1 \%$ F-coverage (Fig. 6.5). The spin filter efficiency is found to be $99.1 \%$, which is slightly smaller than that observed for a $4.1 \%$ F-coverage. For a longer channel length of $\sim 2 \mathrm{~nm}$ (with $8.2 \%$ F-coverage), the spin filter efficiency is found to be $\sim 1$; the transmission from the up state at the Fermi energy completely vanishes. Thus, our result of high spin filter efficiency is valid for a reasonably small F-coverage irrespective of channel length.

To simulate a possible defect in the experimental situation, we have removed one fluorine 


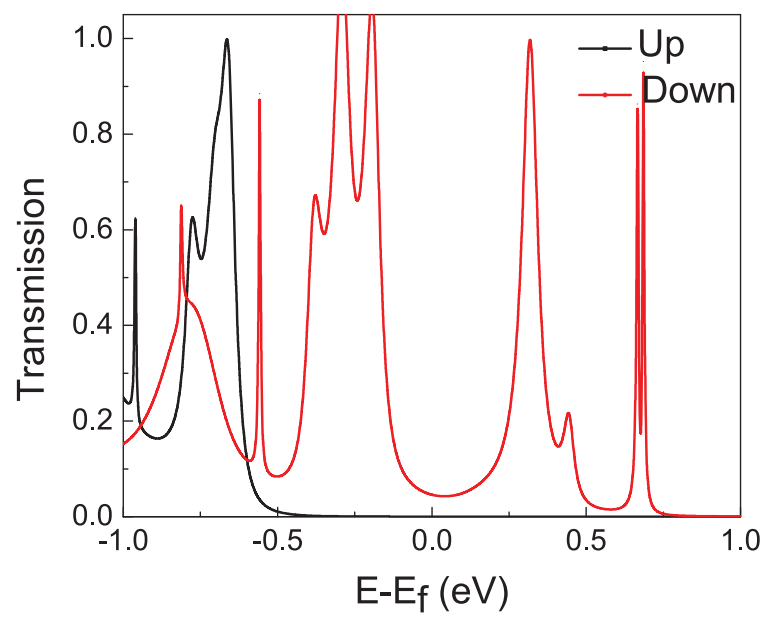

Figure 6.7: Spin dependent transmission in the F-BNNTQD-Au junction; F-coverage is $8.2 \%$ and channel length is $\sim 1.5 \mathrm{~nm}$; Up and Down refer to majority and minority spin states, respectively. Reprinted figure with permission from K. B. Dhungana and R. Pati, J. Am. Chem. Soc. 136, 11494-11498 (2014). (c) The American Chemical Society.

atom from the F-BNNT with a $8.2 \%$ F-coverage (12.5\% defect) and have recalculated the spin dependent transmission (Fig. 6.8). It should be noted that the FM ordering of spins is found to be the most stable configuration for this defect state with a barrier height of 0.11 $\mathrm{eV}$ between the FM and AFM states. From Fig. 6.8, though there is a significant change in the transmission feature with defects is observed as expected in a chemisorbed system, the transmission from the spin down states is again found to be significantly higher than that of the spin up states at the Fermi energy similar to that in a defect free F-BNNTQD (Fig.6.7). The spin filter efficiency is found to be $98.7 \%$, which is slightly smaller than that observed for the defect free F-BNNTQD. The spin injection efficiency has been reported to depend sensitively on the interface; a strongly coupled junction structure has been found to exhibit 


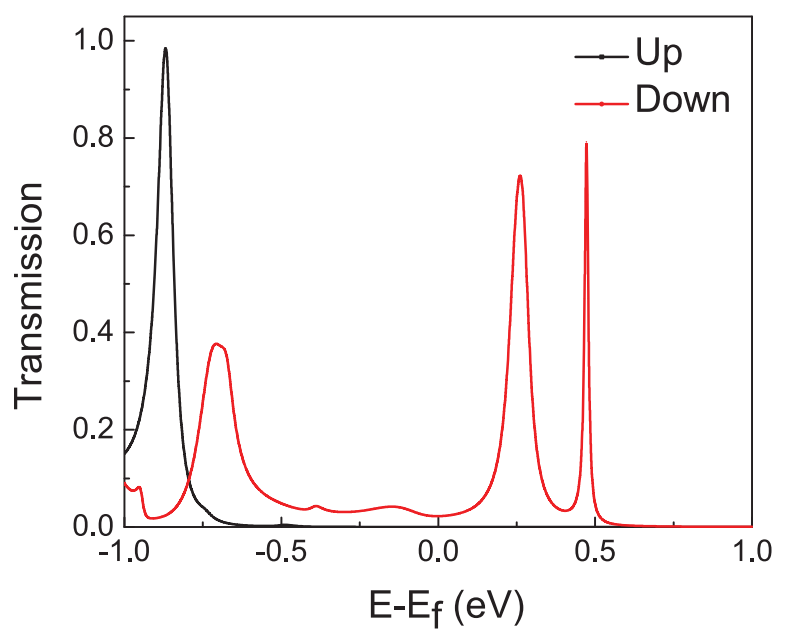

Figure 6.8: Spin dependent transmission in F-BNNTQD-Au junction with a $12.5 \%$ defect; F-coverage is $8.2 \%$ and channel length is $\sim 1.5 \mathrm{~nm}$; Up and Down refer to majority and minority spin states, respectively. Reprinted figure with permission from K. B. Dhungana and Ranjit Pati, J. Am. Chem. Soc. 136, 11494-11498 (2014). (C) The American Chemical Society.

a lower $\eta$ value compared to that in a weakly coupled junction [170]. To check if the F-BNNTQD exhibits such behavior, we have recalculated the spin dependent transmission in a weakly coupled junction structure. To mimic the weak coupling effect at the interface, we have terminated the F-BNNTQD at both interfaces by hydrogen atoms. Our results for the hydrogen terminated F-BNNTQD indeed show a higher spin injection factor of 99.96 $\%$ at the equilibrium, which is in good agreement with the earlier observation [170].

Our analysis of transmission coefficients show that in the case of non-magnetic pristine BNNTQD, no transmission peaks are found around the Fermi energy for the bias range considered here; the transmission values in the vicinity of the Fermi energy is 
found to be very small $\left(8 \times 10^{-5}\right)$. This explains much smaller observed current in the pristine BNNTQD. In contrast, for the magnetic F-BNNTQD, the distinct spin dependent transmission peaks are noticeable (Fig. 6.5). For the up-spin state, the transmission peak, which is closest to the Fermi energy, is observed at $-0.8 \mathrm{eV}$. This transmission peak does not contribute to $I_{\uparrow}$ for the small bias window [ $\left.0,0.4\right]$. However, for the down spin state, a broadened transmission peak appears at the Fermi energy. This leads to an appreciably higher $I_{\downarrow}$ in the case of F-BNNTQD. Within the linear response regime, the conductance

value can be inferred from the transmission data at the Fermi energy as $\mathrm{G}=\frac{e^{2}}{h} T\left(E_{F} ; V=0\right)$. In the case of F-BNNTQD, we find the value of $\mathrm{G}$ to be $3.9 \mu \mathrm{S}$, which is about three order higher than that of the pristine BNNTQD. This change in conductance is not only in good agreement with the experimental report but also reaffirms our previous observation of more than two order of magnitude difference in current between the pristine and fluorinated BNNTQDs. It should be noted that a similar significant change in the conductance value with chemical functionalization of CNT by different molecular adsorbates have been reported $[171,172]$.

\subsubsection{Mechanism for spin filtering in F-BNNT}

To understand the transmission features of the fluorinated BNNTQD at the Fermi-energy, we have analyzed the frontier orbitals of the extended system, which provide the spatial pathway for the spin-polarized charge carriers. For the spin down channel, the highest 
occupied system orbital (HOSO) having contribution from both interfaces (s-orbitals from Au lead and p-orbitals from B and N) contribute to the transmission at the Fermi energy. In contrast, only one interface contributes to $T\left(E_{f}\right)$ for the spin-up channel. A close examination of HOSO for down spin state shows that in addition to the interface states, the p-orbitals of the nitrogen in the close vicinity of adsorbed fluorine atoms in the F-BNNTQD provides the route for spin-down electrons. For the spin-up channel, no such route exists. The broadened feature of the transmission peak at the Fermi energy for the spin-down channel can be understood from the strong coupling between s-states of $\mathrm{Au}$ and $\mathrm{p}$-states of $\mathrm{N}$ and $\mathrm{B}$ at the interfaces. Based on the above discussions, the spin filtering mechanism for
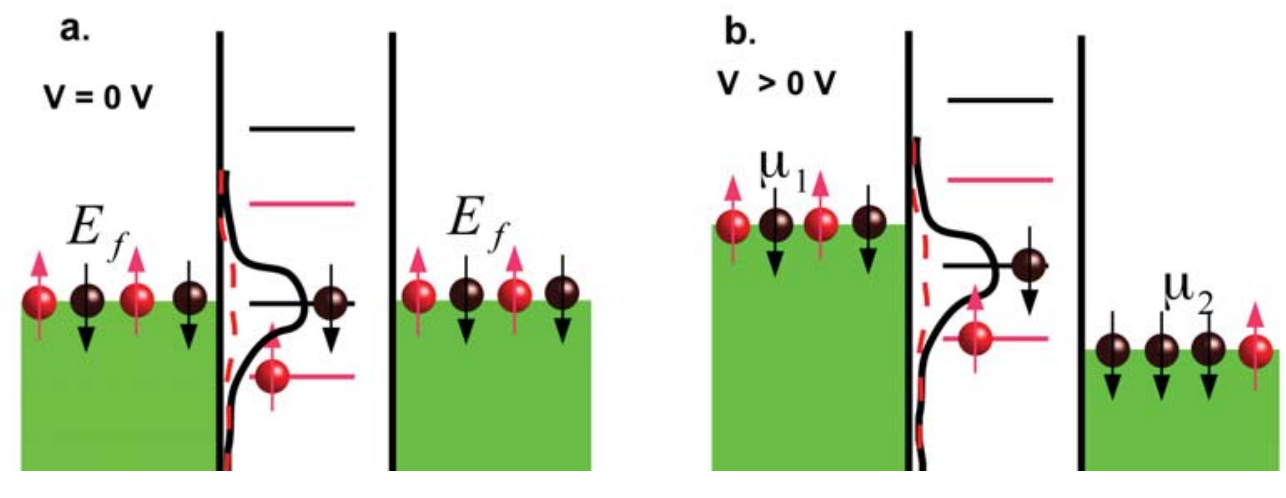

Figure 6.9: Mechanism behind spin filtering in the F-BNNTQD. Horizontal lines represent the position of the discrete spin polarized energy levels of the magnetic F-BNNTQD. Solid smooth curve line represents the density of states (DOS) around the Fermi energy for the spin down states due to coupling with the gold electrodes; dotted line represents the DOS for the spin up states. (a) $\mathrm{V}=0 \mathrm{~V}$ (equilibrium situation), and (b) $\mathrm{V}>0 \mathrm{~V}$. Reprinted figure with permission from K. B. Dhungana and Ranjit Pati, J. Am. Chem. Soc. 136, 11494-11498 (2014). (c) The American Chemical Society. 
the F-BNNTQD can be visualized as shown in Fig. 6.9. Since the F-BNNTQD is magnetic, the energy levels are quantized and spin polarized. When we form the junction between F-BNNTQD and the non-magnetic gold electrodes, the energy levels of the F-BNNTQD for both the up and down spin states redistribute, and broaden with different strength (Fig. 6.9) depending upon the applied bias and the coupling with the interface. The highest occupied molecular orbital (HOMO) of spin down channels not only gets closer to the Fermi energy but also gets broadened significantly in comparison to the HOMO of the spin-up channels during the junction formation; this enhances the escape rate of the spin-down electrons from source to drain, resulting in a significantly higher $I_{\downarrow}$ compared to $I_{\uparrow}$ in the F-BNNTQD.

\subsection{Summary}

Using a first-principles approach, we have shown the conductance of the fluorinated BNNTQD to be more than two order higher than that of the pristine BNNTQD, which is in very good agreement with the experimental report. Furthermore, we predict that the fluorinated BNNT, which exhibits long range ferromagnetic spin ordering at a higher temperature, can be used as an ideal spin filter with efficiency higher than $99 \%$. We expect that these novel findings would generate fresh experimental initiative toward the realization of a next generation spin filter device based upon fluorinated BNNTs. 


\section{Chapter 7}

\section{Conclusions}

In this chapter, I summarize the findings of different projects I completed in my $\mathrm{PhD}$ thesis. For electronic structure study, I have utilized both spin-polarized and spin-unpolarized density functional theory (DFT). To study the quantum (charge \& spin) transport behavior in different nanoscale junctions, I have implemented a single particle Green's function approach together with orbital dependent DFT; particularly, I have used the B3LYP hybrid functional which partly eliminates the self-interaction error. It has been predicted that the B3LYP functional yields better I-V characteristics than other functionals in nanoscale devices.

With the electronic device size approaching its fundamental limit of miniaturization, researchers are relentlessly looking for molecules with rich chemistry to devise new devices 
with novel functionalities. The advantage of using a molecule for a device is that it can be synthesized in large scale without flaws at a cheaper price. Several groups have been successful in demonstrating conduction, rectification, and switching in molecular devices. However, the experimental difficulty of achieving robust atomic level control at the metal-molecule interface hinders the progress in this field. A statistical approach involving measurement of conductance by repeatedly forming thousands of metal-molecule junctions has been used by researchers to extract reliable data for the conductance in the molecular junction. Yet, the qualitative as well as quantitative interpretations of experimental data pose a significant challenge, as the atomic level structural details of the junctions are not available. In addition, the conformations of the molecule as well as the contact structure, which evolve dynamically during experimental measurements, make the theoretical task much harder.

To address these challenges, I have considered an ensemble of device structures by varying metal-molecule binding sites, the orientation of the molecule at the interface, interfacial distances, and conformational change within the molecule to probe the junction dependent effects on conductance in a Ru-bis(terpyridine) (RBT) molecular device, which has been fabricated and characterized experimentally. Based on our calculations, we are able to identify the junction geometry that yields the experimentally measured I-V characteristics; weakly coupled ONTOP junction geometry gives the I-V characteristics that matches reasonably well with the experimentally reported results. 
To explore the possibility of using an organic molecule as a tunnel barrier between two ferromagnetic contacts for application in spin based electronics (spintronics), I have designed a molecular spin valve junction by attaching the same RBT molecule to a nickel contact and have studied the effect of gate field on spin-polarized currents. It should be noted that spintronics relies on the spin state of the electron to store, transport, and process information, and has been the subject of intense research since the discovery of giant magneto-resistance; it helps to alleviate the problem of heat dissipation in nanoscale junctions. Our first-principles quantum transport study based on spin unrestricted density functional theory shows that a modest change in the gate field that is experimentally accessible can lead to a substantial amplification (320\%) of tunnel magnetoresistance (TMR) in RBT/Ni junction-an important requirement for the development of a molecular spin valve transistor.

I have further extended my spin-polarized transport study to a boron nitride nanotube (BNNT) interconnect and have designed a BNNT spin valve transistor based on BNNT quantum dot. The observed giant Stark effect in BNNT inspired me to explore the BNNT spin-valve transistor. For ferromagnetic nickel contacts, we found the exchange coupling and TMR to switch their signs at about the same gate field. The electric field induced modification of magnetic exchange interaction at the interface caused by the Stark effect is found to be the main reason for the observed switching of TMR.

I have also studied the electronic structure, magnetic property and spin-polarized transport 
behavior of fluorine functionalized BNNT, which has been synthesized and characterized. Our first-principles study revealed a significantly higher conductance in F-BNNT than in pristine BNNT, which is in very good agreement with the experimental report. In addition, we predicted that the F-BNNT, which is a metal free ferromagnetic entity, could be used as a perfect spin filter with efficiency as high as 99.8\%; almost all minority spin carriers are found to pass through the F-BNNT channel while blocking all the majority spin carriers. Our density functional calculation shows that the long-range ferromagnetic spin ordering in F-BNNT is stable at a temperature much above the room temperature. 


\section{References}

[1] Moore, G. E. Electronics 1965, 38, 8.

[2] Chau, R.; Doyle, B.; Datta, S.; Kavalieros, J.; Zhang, K. Nature 2007, 6, 810.

[3] Thompson, S. E.; Parthasarathy, S. materialstoday 2006, 9, 20.

[4] Thelander, C.; Bjork, M. T.; Larsson, M. W.; Hansen, A. E.; Wallenberg, L. R.; Samuelson, L. Solid State Communications 2004, 131, 573.

[5] Andergassen, S.; Meden, V.; Schoeller, H.; Splettstoesser, J.; Wegewijs, M. R. Nanotechnology 2010, 21, 272001.

[6] Tao, N. J. nature nanotechnology 2006, 1, 173.

[7] Carroll, R. L.; Gorman, C. B. Angew. Chem. Int. Ed. 2002, 41, 4378.

[8] Wrochem, F.; Gao, D.; Scholz, F.; Nothofer, H.-G.; Nelles, G.; Wessels, J. M. Nature Nanotech. 2010, 5, 618.

[9] Aviram, A.; Ratner, M. A. Chem. Phys. Lett. 1974, 29, 277. 
[10] Reed, M. A.; Zhou, C.; Muller, C. J.; Burgin, T. P.; Tour, J. M. Science 1997, 278, 252.

[11] Xu, B. Q.; Tao, N. J. Science 2003, 301, 1221.

[12] Park, J.; Pasupathy, A. N.; Goldsmith, J. I.; Chang, C.; Yaish, Y.; Petta, J. R.; Rinkoski, M.; Sethna, J. P.; Abruna, H. D.; McEuen, P. L.; Ralph, D. C. Nature 2002, 417, 722 .

[13] Song, B.; Ryndyk, D. A.; Cuniberti, G. Phys. Rev. B 2007, 76, 045404.

[14] Chen, J.; Reed, M. A.; Rawlett, A. M.; Tour, J. M. Science 1999, 286, 1550.

[15] Xu, B.; Xiao, X.; Yang, X.; Zang, L.; Tao, N. J. Am. Chem. Soc 2005, 127, 2386.

[16] Song, H.; Kim, Y.; Jang, Y. H.; Jeong, H.; Reed, M. A.; Lee, T. Nature 2009, 462, 1039.

[17] Xiang, D.; Jeong, H.; Kim, D.; Lee, T.; Cheng, Y.; Wang, Q.; Mayer, D. Nano Lett. 2013, 13, 2809.

[18] Faleev, S. V.; Leonard, F.; Stewart, D. A.; Schilfgaarde, M. Phys. Rev. B 2005, 71, 195422.

[19] Samanta, M. P.; Tian, W.; Datta, S.; Henderson, J. I.; Kubiak, C. P. Phys. Rev. B 1996, 53, R7626.

[20] Emberly, E. G.; Kirczenow, G. Phys. Rev. B 1999, 61, 5740. 
[21] Yaliraki, S. N.; Roitberg, A. E.; Gonzalez, C.; Mujica, V.; Ratner, M. A. J. Chem. Phys. 1999, 111, 6997.

[22] Delaney, P.; Ventra, M. D.; Pantelides, S. T. App. Phys. Lett. 1999, 75, 3787.

[23] Pati, R.; Karna, S. P. Phys. Rev. B 2004, 69, 155419.

[24] Datta, S. Electron Transport in Mesoscopic Systems; Cambridge University Press: Cambridge, UK,, 1997.

[25] Ventra, M. D. Electrical Transport in Nanoscale Systems; Cambridge: New York, 2008.

[26] Pati, R.; McClain, M.; Bandyopadhyay, A. Phys. Rev. Lett. 2008, 100, 246801.

[27] Xue, Y.; Datta, S.; Ratner, M. A. Chem. Phys. 2002, 281, 151.

[28] Brandbyge, M.; Mozos, J.-L.; P. Ordejon, J. T.; Stokbro, K. Phys. Rev. B 2002, 65, 165401.

[29] Dhungana, K. B.; Mandal, S.; Pati, R. J. Phys. Chem. C 2012, 116, 17268.

[30] Wolf, S. A.; Awschalom, D. D.; Buhrman, R. A.; Daughton, J. M.; von Molnar, S.; Roukes, M. L.; Chtchelkanova, A. Y.; Treger, D. M. Science 2001, 294, 1488.

[31] Zutic, I.; Fabian, J.; Sarma, S. D. Rev. Mod. Phys. 2004, 76, 323.

[32] Chappert, C.; Fert, A.; Dau, F. N. Nat. Material 2007, 6, 813.

[33] Fert, A. Rev. Mod. Phys. 2008, 80, 1517. 
[34] Binasch, G.; Grunberg, P.; Saurenbach, F.; Zinn, W. Phys. Rev. B 1989, 39, 4828R.

[35] Baibich, M. N.; Broto, J. M.; Fert, A.; Dau, F. N. V.; Petroff, F.; Etienne, P.; Creuzet, G.; Friederich, A.; Chazelas, J. Phys. Rev. Lett. 1988, 61, 2472.

[36] Parkin, S. S. P.; Kaiser, C.; Panchula, A.; Rice, P. M.; Hughes, B.; Samant, M.; Yang, S.-H. Nat. Material 2004, 3, 862.

[37] Bader, S. D.; Parkin, S. S. P. Annu. Rev. Condens. Matter Phys. 2010, 1, 71.

[38] Mizuno, Y.; Ohya, S.; Hai, P. N.; Tanaka, M. App. Phys. Lett. 2007, 90, 162505.

[39] Sanvito, S. Chem. Soc. Rev. 2011, 90, 3336.

[40] Tsukagoshi, K.; Alphenaar, B. W.; Ago, H. Nature 1999, 401, 572.

[41] Kawahara, S. L.; Lagoute, J.; Repain, V.; Chacon, C.; Girard, Y.; Rousset, S.; Smogunov, A.; Barreteau, C. Nano Lett. 2012, 12, 4558.

[42] Yamada, R.; Noguchi, M.; Tada, H. App. Phys. Lett. 2011, 98, 053110.

[43] Schmaus, S.; Bagrets, A.; Nahas, Y.; Yamada, T. K.; Bork, A.; Bowen, M.; Beaurepaire, E.; Evers, F.; Wulfhekel, W. Nat. Nanotech. 2010, 6, 185.

[44] Bagrets, A.; Schmaus, S.; Jaafar, A.; Kramczynski, D.; Yamada, T. K.; Alouani, M.; Wulfhekel, W.; Evers, F. Nano Lett. 2012, 12, 5131.

[45] Horiguchi, K.; Sagisaka, T.; Kurokawa, S.; Sakai, A. J. Appl. Phys. 2013, 113, 144313. 
[46] Yoshida, K.; Hamada, I.; Sakata, S.; Umeno, A.; Tsukada, M.; Hirakawa, K. Nano Lett. 2013, 13, 481.

[47] Waldron, D.; Haney, P.; Larade, B.; MacDonald, A.; Guo. Phys. Rev. Lett. 2006, 96, 166804.

[48] Rocha, A. R.; Garcia-suarez, V. M.; Bailey, S. W.; Lambert, C. J.; Ferrer, J.; Sanvito, S. Nat. materials 2005, 4, 335 .

[49] Dhungana, K. B.; Pati, R. App. Phys. Lett 2014, 104, 162404.

[50] Datta, S.; Das, B. App. Phys. Lett 1990, 56, 665.

[51] Zeng, J.; Chen, K.-Q. J. Mat. Chem. C 2013, 1, 4014.

[52] Dhungana, K. B.; Pati, R. J. Am. Chem. Soc. 2014, 136, 11494.

[53] Bogani, L.; Wernsdorfer, W. Nat. Mat. 2008, 7, 179.

[54] Moodera, J. S.; Santos, T. S.; Nagahama, T. J. Phys. Condens. Mat. 2007, 19, 165202.

[55] Sanvito, S. Nat. Mater. 2011, 10, 484.

[56] Zhou, Y.-H.; Zeng, J.; Tang, L.-M.; Chen, K.-Q.; Hu, W. P. Org. Electron 2013, 14, 2940.

[57] Sen, S.; Chakrabarti, S. J. Am. Chem. Soc. 2010, 132, 15334. 
[58] Barraza-Lopez, S.; Park, K.; Suarez, V. G.; Ferrer, J. Phys. Rev. Lett. 2009, 102, 246801.

[59] Muller, M.; Miao, G.-X.; Moodera, J. M. Europhys. Lett. 2009, 88, 47006.

[60] Nagahama, T.; Santos, T. S.; Moodera, J. S. Phys. Rev. Lett 2007, 99, 016602.

[61] Makarova, T. L. Semiconductors 2004, 38, 641.

[62] Capelle, K. arXiv:cond-mat/0211443.

[63] Parr, R. G.; Yang, W. Density Functional Theory of Atoms and Molecules; Oxford University Press, New York, 1989.

[64] Szabo, A.; Ostlund, N. S. Modern Quantum Chemistry; Dover Publishingm Mineola, New York, 1996.

[65] Hohenberg, P.; Kohn, W. Phy. Rev. 1964, 136, 864.

[66] Kohn, W. Mod. Phys. 1999, 71, 1253.

[67] Kohn, W.; Sham, L. J. Phys. Rev. 1965, 140, A1133.

[68] Payne, M. C.; Teter, M. P.; Ailan, D. C.; Arias, T. A.; Joannopouios, J. D. Rev. Mod. Phys. 1992, 64, 1045.

[69] Burke, K. J. Chem. Phys. 2012, 136, 150901.

[70] Becke, A. D. J. Chem. Phys. 2014, 140, 18A301. 
[71] Perdew, J. P.; Burke, K.; Wang, Y. Phys. Rev. B 1996, 54, 16533.

[72] Oliveira, L. N.; Gross, E. K. U.; Kohn, W. Phys. Rev. Lett. 1988, 60, 2430.

[73] Carloni, P.; Rothlisberger, U.; Parrinello, M. Acc. Chem. Res. 2002, 35, 455.

[74] Pati, R. Theory of Electronic Structures and Nuclear Quadrupole Interactions in Molecular Solids and Semiconductors Surfaces $\mathrm{PhD}$ thesis, State University of New York, 1998.

[75] Roothaan, C. C. J. Rev. Mod. Phys. 1951, 23, 69.

[76] Hartree, D. R. The calculation of atomic structure; John Wiley and Sons, New York, 1957.

[77] Born, M.; Oppenheimer, J. R. Ann. Physik. 1927, 84, 457.

[78] Harrison, N. M. An introduction to density functional theory; Catlow, Kotomin (Eds.), Computational materials science NATO science series III, IOS Press, Berlin, p. $187,2003$.

[79] Thomas, L. H. Math Proc. Camb. Phil. Soc. 1927, 23, 542.

[80] Honenberg, P.; Kohn, W. Phys. Rev. 1994, 136, 864.

[81] Perdew, J. P.; Burke, K.; Ernzerhof, M. Phys. Rev. Lett. 1996, 77, 3865.

[82] Perdew, J. P.; Burke, K. Int. J. Quan. Chem. 1996, 57, 309.

[83] Grimme, S. J. Comp. Chem. 2006, 27, 1787. 
[84] Cramer, C. J.; truhlar, D. G. Phys. Chem. Chem. Phys. 2009, 11, 1075710816.

[85] Xiao, H.; Tahir-Kheli, J.; Goddard, W. A. Phys. Chem. Lett. 2011, 2, 212.

[86] Perger, W. F. Chem. Phys. Lett. 2003, 368, 319323.

[87] Pal, P. P. Quantum transport in a single molecular junction $\mathrm{PhD}$ thesis, Michigan Tech. University, 2011.

[88] Sahoo, S. An Experimental Investigation of Spin Polarized Transport in Carbon Nanotubes $\mathrm{PhD}$ thesis, University of Basel, 2005.

[89] Landauer, R. IBM J. Res. Rev. 1957, 1, 223.

[90] Landauer, R. J. Phys. Cond. Matt. 1989, 1, 8099.

[91] Pal, P. P.; Pati, R. Phys. Rev. B 2010, 82, 045424.

[92] Mandal, S.; Pati, R. ACS Nano 2012, 6, 3580.

[93] Donhauser, Z. J.; Mantooth, B. A.; Kelly, K. F.; Bumm, L. A.; Monnell, J. D.; Stapleton, J. J.; Price, D. W.; Rawlett, A. M.; Allara, D. L.; Tour, J. M.; Weiss, P. S. Science 2001, 292, 2303.

[94] Zhou, J.; Chen, F.; Xu, B. Phys. Lett. A 2009, 131, 10439.

[95] Hihath, J.; Bruot, C.; Nakamura, H.; Asai, Y.; Dez-Perez, I.; Lee, Y.; Yu, L.; Tao, N. J. ACS Nano 2011, 5, 8331.

[96] Nitzan, A.; Ratner, M. A. Science 2003, 300, 1384. 
[97] Cui, X. D.; Primak, A.; Zarate, X.; Tomfohr, J.; Sankey, O. F.; Moore, A. L.; Moore, T. A.; Gust, D.; Harris, G.; Lindsay, S. M. Science 2001, 294, 571.

[98] Huang, Z.; Chen, F.; Bennett, P. A.; Tao, N. J. J. Am. Chem. Soc. 2007, 129, 13225.

[99] Hu, Y.; Zhu, Y.; Guo, H. Phys. Rev. Lett. 2005, 95, 156803.

[100] French, W. R.; Lacovella, C. R.; Cummings, P. T. ACS Nano 2012, 6, 2779.

[101] Pontes, R. B.; Rocha, A. R.; Sanvito, S.; Fazzio, A.; Silva, A. J. R. ACS Nano 2011, $5,795$.

[102] Andrews, D. Q.; Duyne, R. P. V.; Ratner, M. A. Nano Lett. 2008, 8, 1120.

[103] Slonczewski, J. C. Phys. Rev. B 1989, 39, 6995.

[104] Julliere, M. Phys. Lett. A 1975, 54, 225.

[105] Moodera, J. S.; Kinder, L. R.; Wong, T. M.; Meservey, R. Phys. Rev. Lett. 1995, 74, 3273.

[106] Ning, Z.; Zhu, Y.; Wang, J.; Guo, H. Phy. Rev. Lett. 2008, 100, 056803.

[107] Pati, R.; Senapati, L.; Ajayan, P. M.; Nayak, S. K. Phy. Rev. B 2003, 68, 1004071.

[108] Pasupathy, A. N.; Bialczak, R. C.; Martinek, J.; Grose, J. E.; Donev, L. A. K.; McEuen, P. L.; Ralph, D. C. Science 2004, 306, 86.

[109] Zwolak, M.; Ventra, M. D. Phy. Rev. Lett. 2002, 81, 925. 
[110] Zhou, J. G.; Hagelberg, F. Phys. Rev. Lett. 2006, 97, 045505.

[111] Seo, K.; Konchenko, A. V.; Lee, J.; Bang, G. S.; Lee, H. J. Am. Chem. Soc. 2008, $130,2553$.

[112] Taylor, J.; Guo, H.; Wang, J. Phys. Lett. B 2001, 63, 245407.

[113] Toher, C.; Sanvito, S. Phys. Rev. Lett. 2008, 99, 056801.

[114] Brandbyge, M.; Mozos, J. L.; Ordejon, P.; Taylor, J.; Stokbro, K. Phys. Lett. B 2002, $65,165401$.

[115] Xue, Y.; Datta, S.; Ratner, M. A. J. Chem. Phys. 2001, 115, 4292.

[116] Su, W.; Jiang, J.; Lu, W.; Luo, Y. Nano Lett. 2006, 6, 2091.

[117] Solomon, G. C.; Herrmann, C.; Hansen, T.; Mujica, V.; Ratner, M. A. Nat. Chem 2010, 2, 223.

[118] Mandal, S.; Pati, R. Phys. Rev. B 2011, 84, 115306.

[119] 03, G. Revision C.02; Gaussian Inc.: Pittsburgh, PA, 2003.

[120] Becke, A. D. J. Chem. Phys. 1993, 98, 5648.

[121] Runge, E.; Gross, E. K. U. Phys. Rev. Lett 1984, 52, 997.

[122] Evers, F.; Weigend, F.; Koentopp, M. Phys. Rev. B 2004, 69, 235411.

[123] Sai, N.; Zwolak, M.; Vignale, G.; Ventra, M. D. Phys. Rev. Lett. 2005, 94, 186810. 
[124] Vignale, G.; Ventra, M. D. Phys. Rev. B 2009, 79, 014201.

[125] Pati, R.; Karna, S. P. Phys. Rev. B 2004, 69, 155419.

[126] GAUSSIAN09. revision A.1; Gaussian, Inc., Wallingford, CT, 2009.

[127] Herrmann, C.; Solomon, G. C.; Ratner, M. A. J. Am. Chem. Soc. 2010, 132, 3682.

[128] Ouyang, M.; Awschalom, D. D. Science 2003, 301, 1074.

[129] Hauptmann, J. R.; Paaske, J.; Lindelof, P. E. Nat. Phys. 2008, 4, 373.

[130] Sahoo, S.; Kontos, T.; Furer, J.; Hoffmann, C.; Graber, M.; Cottet, A.; Schonenberger, C. Nat. Phys. 2005, 1, 99.

[131] Urdampilleta, M.; Klyatskaya, S.; Cleuziou, J.-P.; Ruben, M. Nat. Mater 2011, 10, 502.

[132] Barraud, C.; Seneor, P.; Mattana, R.; Fusil, S.; Bouzehouane, K.; Deranlot.; Graziosi, P.; Hueso, L.; Bergenti, I.; Dediu, V.; Petroff, F.; Fert, A.

[133] Yamada, R.; Noguchi, M.; Tada, H. J. Appl. Phys 2013, 113, 144313.

[134] Parkin, S. S. P.; More, N.; Roche, K. P. Phys. Rev. Lett. 1990, 64, 2304.

[135] Dediu, V. A.; Hueso, L.; Bergenti, I.; Taliani, C. Phys. Rev. Lett. 2009, 8, 707.

[136] Kim, W. Y.; Kim, K. Acc. Chem. Res. 2010, 43, 111.

[137] D. Liu, Y. Hu, H. G.; Han, X. F. Phys. Rev. B 2008, 78, 193307. 
[138] Atodiresei, N.; Brede, J.; Lazic, P.; Caciuc, V.; Hoffmann, G.; Wiesendanger, R.; Blugel, S. Phys. Rev. Lett. 2010, 105, 066601.

[139] Sanvito, S. Nat. Phys. 2010, 6, 562.

[140] Ruden, P. Nat. Mater. 2011, 10, 8.

[141] Wang, B.; Zhu, Y.; Ren, W.; Wang, J.; Guo, H. Phys. Rev. B 2007, 75, 235415.

[142] Golberg, D.; Bando, Y.; Huang, Y.; Terao, T.; Mitome, M.; Tang, C.; Zhi, C. ACS Nano 2010, 4, 2979.

[143] Chopra, N. G.; Luyken, R. J.; Cherrey, K.; Crespi, V. H.; Cohen, M. L.; Louie, S. G.; Zettl, A. Science 1995, 269, 966.

[144] Ishigami, M.; Sau, J. D.; Aloni, S.; Cohen, M. L.; Zettl, A. Phys. Rev. Lett. 2005, 94, 056804.

[145] Ventra, M. D.; Pantelides, S. T.; Lang, N. Phys. Rev. Lett. 2000, 84, 979.

[146] Zwolak, M.; Ventra, M. D. App. Phys. Lett. 2002, 81, 925.

[147] Dalgleish, H.; Kirczenow, G. App. Phys. B 2005, 72, 184407.

[148] Khoo, K. H.; Mozzoni, M. S. C.; Louie, S. G. App. Phys. B 2004, 69, 201401.

[149] Chen, C.-W.; Lee, M.-H.; Clark, S. J. Nanotechnology 2004, 15, 1837.

[150] Kong, J.; Yenilmez, E.; Thomas, W.; Kim, T. W.; Dai, H.; Laughlin, R. B.; Liu, L.; Jayanthi, C. S.; Wu, S. Y. Phy. Rev. Lett. 2001, 87, 106801. 
[151] Cinchetti, M.; Heimer, K.; Wustenberg, J.-P.; Andreyev, O.; Bauer, M.; Lach, S.; Ziegler, C.; Gao, Y.; Aeschlimann, M. Nat. Mater 2009, 8, 115.

[152] Rubio, A.; Corkill, J. L.; Cohen, M. L. Phys. Rev. B 1994, 49, 5081.

[153] Blase, X.; Rubio, A.; Louie, S. G.; Cohen, M. L. Euro. Phys. Lett. 1994, 28, 335.

[154] Iijima, S. Nature 1991, 56, 354.

[155] Dhungana, K. B.; Pati, R. Sensors 2014, 14, 17655.

[156] Zhi, C. Y.; Bando, Y.; Tang, C. C.; Huang, Q.; Golberg, D. J. Mat. Chem. 2008, 18, 3900.

[157] Tang, C.; Bando, Y.; Huang, Y.; Yue, S.; Gu, C.; Xu, F.; Golberg, D. J. Am. Chem. Soc 2005, 127, 6552.

[158] Zhao, J.; Li, W.; Tang, C.; Li, L.; Lin, J.; Gu, C. Appl. Phys. Lett. 2013, 102, 153107.

[159] Li, F.; Zhu, Z.; Yao, X.; Lu, G.; Zhao, M.; Xia, Y.; Chen, Y. Appl. Phys. Lett. 2008, $92,102515$.

[160] Zhang, Z.; Guo, W. J. Am. Chem. Soc. 2009, 131, 6874.

[161] Dhungana, K. B.; Pati, R. Phys. Chem. Chem. Phys. 2014, 16, 7996.

[162] Makarova, T. L. Nature 2001, 413, 716.

[163] Terauchi, M.; Tanaka, M.; Suzuki, K.; Ogino, A.; Kimurab, K. Chem. Phys. Lett. 2000, 324, 359. 
[164] Golberg, D.; Bando, Y.; Bourgeois, L.; Kurashima, K.; Sato, T. Appl. Phys. Lett. 2000, 77, 1979.

[165] Kresse, G.; Furthmüller, J. Phys. Rev. B 1996, 54, 11169.

[166] Kinoshita, Y.; Ohno, N. Phys. Rev. B 2010, 82, 085433.

[167] Zhou, Z.; Zhao, J.; Chen, Z.; Schleyer, P. R. J. Phys. Chem. B 2006, 110, 25678.

[168] Park, K. A.; Choi, Y. S.; Lee, Y. H.; Kim, C. Phys. Rev. B 2003, 68, 045429.

[169] Zhou, J.; Wang, Q.; Sun, Q.; Jena, P. Phys. Rev. B 2010, 81, 085442.

[170] Kondo, H.; Ohno, T. Phys. Rev. Lett. 2013, 103, 233115.

[171] García-Lastra, J. M.; Thygesen, K. S.; Strange, M.; Rubio, A. Phys. Rev. Lett. 2008, $101,236806$.

[172] García-Lastra, J. M.; Mowbray, D. J.; Thygesen, K. S.; Rubio, A.; Jacobsen, K. W. Phys. Rev. B 2010, 81, 245429.

[173] Tang, C.; Bando, Y.; Sato, T.; Kurashima, K. Chem. Commun. 2002, 12, 1290.

[174] Fukushima, T.; Kosaka, A.; Ishimura, Y.; Yamamoto, T.; Takigawa, T.; Ishii, N.; Aida, T. Science 2003, 300, 2072.

[175] Han, W.-Q.; Zettl, A. J. Am. Chem. Soc. 2003, 125, 2062.

[176] Mukhopadhyay, S.; Gowtham, S.; Scheicher, R. H.; Pandey, R.; Karna, S. P. Nanotechnology 2010, 21, 165703. 
[177] Mukhopadhyay, S.; Scheicher, R. H.; Pandey, R.; Karna, S. P. J. Phys. Chem. Lett. 2011, 2, 2442.

[178] Lau, Y.-T. R.; Yamaguchi, M.; Li, X.; Bando, Y.; Golberg, D.; Winnik, F. M. J. Phys. Chem. C 2013, 117, 19568. 



\section{Appendix A}

\section{Copyrights}

The copyright permission from the American Chemical Society for the article by K. B.

Dhungana, S. Mandal, and R. Pati, J. Phys. Chem. C 116, 17268-17273 (2012). The permission applies to Chapter 4 .

The copyright permission from the American Institute of Physics for the article by K. B. Dhungana and R. Pati, App. Phys. Lett. 104, 16204-16207 (2014). The permission applies to Chapter 4. 


\section{Copyright Clearance \\ Center

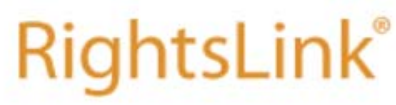

ACSPublications ritle:

Most Trusted. Most Cited. Most Read.
Switching of Conductance in a Molecular Wire: Role of Junction Geometry, Interfacial Distance, and Conformational Change

Author: Kamal B. Dhungana, Subhasish Mandal, Ranjit Pati

Publication: The Journal of Physical Chemistry $\mathrm{C}$

Publisher: American Chemical Society

Date: $\quad$ Aug 1, 2012

Copyright (c) 2012, American Chemical Society

\section{PERMISSION/LICENSE IS GRANTED FOR YOUR ORDER AT NO CHARGE}

This type of permission/license, instead of the standard Terms \& Conditions, is sent to you because no fee is being charged for your order. Please note the following:

- Permission is granted for your request in both print and electronic formats, and translations.

- If figures and/or tables were requested, they may be adapted or used in part.

- Please print this page for your records and send a copy of it to your publisher/graduate school.

- Appropriate credit for the requested material should be given as follows: "Reprinted (adapted) with permission from (COMPLETE REFERENCE CITATION). Copyright (YEAR) American Chemical Society." Insert appropriate information in place of the capitalized words.

- One-time permission is granted only for the use specified in your request. No additional uses are granted (such as derivative works or other editions). For any other uses, please submit a new request.

\section{BACK}

CLOSE WINDOW

Copyright () 2015 Copyriaht Clearance Center, Inc. All Rights Reserved. Privacy statement. Terms and Conditions. Comments? We would like to hear from you. E-mail us at customercare@copyriaht.com

Figure A.1: Copyright permission from the American Chemical Society for the article by K. B. Dhungana, S. Mandal, and R. Pati, J. Phys. Chem. C 116, 17268-17273 (2012). 


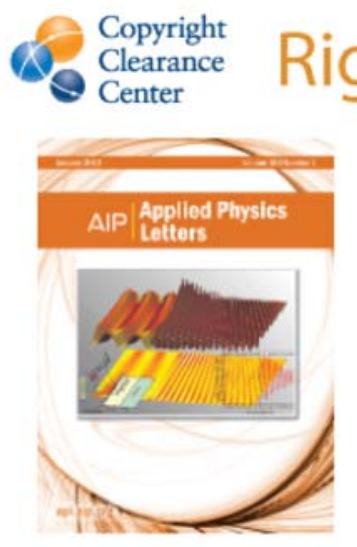

ghtsLink

Title:

Giant amplification of tunnel

magnetoresistance in a molecular junction: Molecular spin-valve transistor

Author:

Kamal B. Dhungana,Ranjit

Pati

Publication: Applied Physics Letters

Volume/Issue 104/16

Publisher: AIP Publishing LLC

Date: $\quad$ Apr 22, 2014

Page Count: 4

Rights managed by AIP Publishing LLC.
Logged in as:

Kamal Dhungana

Account \#:

3000780990

LOGOUT

\section{Order Completed}

Thank you very much for your order.

Click here for Payment Terms and Conditions.

Get a printable version for your records.

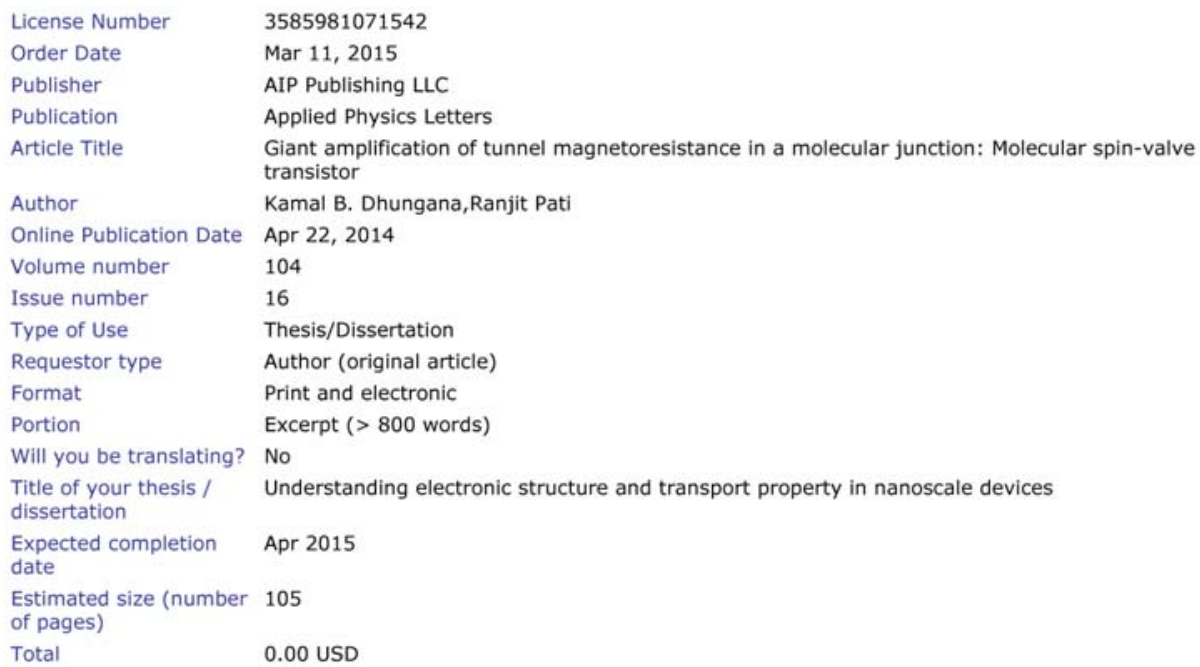

Copyright (c) 2015 Copyriaht Clearance Center, Inc. All Rights Reserved. Privacy statement. Terms and Conditions. Comments? We would like to hear from you. E-mail us at customercare@copyriaht.com

Figure A.2: Copyright permission from the American Institute of Physics for the article by K. B. Dhungana and R. Pati, App. Phys. Lett. 104, 16204-17207 (2014). 


\section{Appendix B}

\section{Copyrights}

The copyright permission from the Royal Society of Chemisty for the article by K. B.

Dhungana and R. Pati, Phys. Chem. Chem. Phys. 16, 7996-8002 (2014). The permission applies to Chapter 5 . 


\section{Electrical tuning of spin current in a boron nitride nanotube quantum dot}

K. B. Dhungana and R. Pati, Phys. Chem. Chem. Phys., 2014, 16, 7996

DOI: $10.1039 / \mathrm{C} 4 \mathrm{CP} 00325 \mathrm{~J}$

If you are not the author of this article and you wish to reproduce material from it in a third party nonRSC publication you must formally request permission using RightsLink. Go to our Instructions for using RightsLink page for details.

Authors contributing to RSC publications (journal articles, books or book chapters) do not need to formally request permission to reproduce material contained in this article provided that the correct acknowledgement is given with the reproduced material.

Reproduced material should be attributed as follows:

- For reproduction of material from NJC:

Reproduced from Ref. XX with permission from the Centre National de la Recherche Scientifique

(CNRS) and The Royal Society of Chemistry.

- For reproduction of material from PCCP:

Reproduced from Ref. XX with permission from the PCCP Owner Societies.

- For reproduction of material from PPS:

Reproduced from Ref. XX with permission from the European Society for Photobiology, the

European Photochemistry Association, and The Royal Society of Chemistry.

- For reproduction of material from all other RSC journals and books:

Reproduced from Ref. XX with permission from The Royal Society of Chemistry.

If the material has been adapted instead of reproduced from the original RSC publication "Reproduced from" can be substituted with "Adapted from".

In all cases the Ref. $\mathrm{XX}$ is the $\mathrm{XX}$ th reference in the list of references.

If you are the author of this article you do not need to formally request permission to reproduce figures, diagrams etc. contained in this article in third party publications or in a thesis or dissertation provided that the correct acknowledgement is given with the reproduced material.

Reproduced material should be attributed as follows:

- For reproduction of material from NJC:

[Original citation] - Reproduced by permission of The Royal Society of Chemistry (RSC) on behalf of the Centre National de la Recherche Scientifique (CNRS) and the RSC

- For reproduction of material from PCCP:

[Original citation] - Reproduced by permission of the PCCP Owner Societies

- For reproduction of material from PPS:

[Original citation] - Reproduced by permission of The Royal Society of Chemistry (RSC) on behalf of the European Society for Photobiology, the European Photochemistry Association, and RSC

- For reproduction of material from all other RSC journals:

[Original citation] - Reproduced by permission of The Royal Society of Chemistry

If you are the author of this article you still need to obtain permission to reproduce the whole article in a third party publication with the exception of reproduction of the whole article in a thesis or dissertation.

Figure B.1: Copyright permission from the American Chemical Society for the article by K. B. Dhungana and R. Pati, Phys. Chem. Chem. Phys. 16, 7996-8002 (2014). 


\section{Appendix C}

\section{Copyrights}

The copyright permission from the American Chemical Society for the article by K. B.

Dhungana and R. Pati, J. Am. Chem. Soc. 136, 11494-11498 (2014). The permission applies to Chapter 6. 


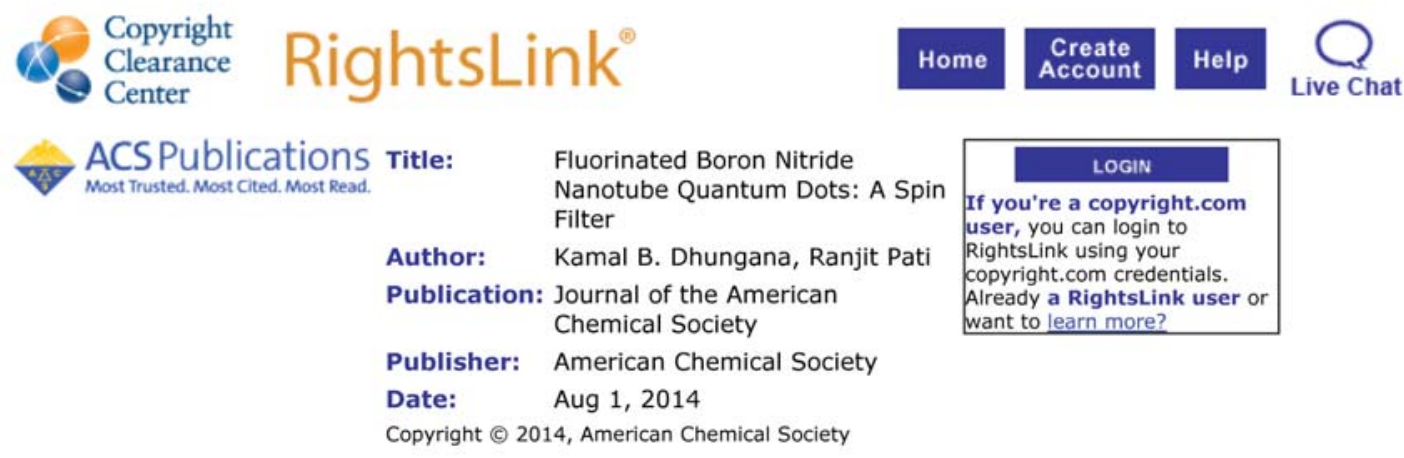

PERMISSION/LICENSE IS GRANTED FOR YOUR ORDER AT NO CHARGE

This type of permission/license, instead of the standard Terms \& Conditions, is sent to you because no fee is being charged for your order. Please note the following:

- Permission is granted for your request in both print and electronic formats, and translations.

- If figures and/or tables were requested, they may be adapted or used in part.

- Please print this page for your records and send a copy of it to your publisher/graduate school.

- Appropriate credit for the requested material should be given as follows: "Reprinted (adapted) with permission from (COMPLETE REFERENCE CITATION). Copyright (YEAR) American Chemical Society." Insert appropriate information in place of the capitalized words.

- One-time permission is granted only for the use specified in your request. No additional uses are granted (such as derivative works or other editions). For any other uses, please submit a new request.

\section{BACK CLOSE WINDOW}

Copyright (c) 2015 Copyriaht Clearance Center, Inc., All Rights Reserved. Privacy statement. Terms and Conditions. Comments? We would like to hear from you. E-mail us at customercare@copyriaht.com

Figure C.1: Copyright permission from the American Chemical Society for the article by K. B. Dhungana and R. Pati, J. Am. Chem. Soc. 136, 11494-11498 (2014). 